\title{
Four-dimensional ambitwistor strings and form factors of local and Wilson line operators
}

\author{
L. V. Bork ${ }^{1,2,3}$ and A. I. Onishchenko ${ }^{4,5,6}$ \\ ${ }^{1}$ Institute for Theoretical and Experimental Physics, Moscow 117218, Russia \\ ${ }^{2}$ National Research Nuclear University (MEPhI), Moscow 115409, Russia \\ ${ }^{3}$ The Center for Fundamental and Applied Research, All-Russia Research Institute of Automatics, \\ Moscow 117055, Russia \\ ${ }^{4}$ Bogoliubov Laboratory of Theoretical Physics, Joint Institute for Nuclear Research, Dubna 141980, Russia \\ ${ }^{5}$ Moscow Institute of Physics and Technology (State University), Dolgoprudny 141701, Russia \\ ${ }^{6}$ Skobeltsyn Institute of Nuclear Physics, Moscow State University, Moscow 119991, Russia
}

(Received 21 February 2018; published 20 June 2018)

\begin{abstract}
We consider the description of form factors of local and Wilson line operators (Reggeon amplitudes) in $\mathcal{N}=4$ Supersymmetric Yang-Mills theory within the framework of four-dimensional ambitwistor string theory. We present the explicit expressions for string composite operators corresponding to stress-tensor operator supermultiplet and Wilson line operator insertion. It is shown that corresponding tree level string correlation functions correctly reproduce previously obtained Grassmannian integral representations. As a by-product we derive four-dimensional tree-level scattering equation representations for the mentioned form factors and formulate a simple gluing procedure used to relate operator form factors with on-shell amplitudes.
\end{abstract}

DOI: $10.1103 /$ PhysRevD.97.126013

\section{INTRODUCTION}

Recently twistor string theories [1,2] played a crucial role in understanding and discovery of mathematical structures underlying scattering amplitudes in $\mathcal{N}=4$ super Yang-Mills and $\mathcal{N}=8$ supergravity in four dimensions.

Based on Witten's twistor string theory Roiban, Spradlin, and Volovich (RSV) got the integral representation of $\mathcal{N}=4$ Supersymmetric Yang-Mills theory (SYM) tree level $\mathrm{N}^{k-2}$ MHV amplitudes as integrals over the moduli space of degree $k-1$ curves in super twistor space $[3,4]$. Further generalization of the RSV result was performed by Cachazo, $\mathrm{He}$ and Yuan $(\mathrm{CHY})$ via the introduction of so-called scattering equations [5-9]. Within the latter $\mathcal{N}=4 \mathrm{SYM}$ amplitudes are expressed in terms of integrals over the marked points on the Riemann sphere, which are localized on the solutions of mentioned scattering equations. Next the CHY formulas were shown to come naturally from ambitwistor string theory [10,11], which was also used to obtain loop-level generalization of scattering equation representation [12-21]. Another close direction in the study of scattering amplitudes is related to their representation in terms of integrals over Grassmannians [22-27].

Published by the American Physical Society under the terms of the Creative Commons Attribution 4.0 International license. Further distribution of this work must maintain attribution to the author(s) and the published article's title, journal citation, and DOI. Funded by SCOAP ${ }^{3}$.
First, this representation allows natural unification of different Britto-Cachazo-Feng-Witten (BCFW) [28,29] representations for tree level amplitudes and loop level integrands $[22,23]$. Second, it is ultimately related to the integrable structure behind the $\mathcal{N}=4$ SYM $S$ matrix [30-34]. Moreover, the Grassmannian integral representation also naturally relates perturbative $\mathcal{N}=4 \mathrm{SYM}$ and twistor string theories amplitudes[26]. Finally, the Grassmannian integral representation of scattering amplitudes has led to the discovery of the geometrical structure of the $\mathcal{N}=4$ SYM $S$ matrix (so-called amplituhedron) [35-42].

All results mentioned above are extremely relevant not only from a pure theoretical but also from a more practical point of view. For example, these results provide us with relatively compact analytical expressions for $n$-point tree level amplitudes in gauge theories with $\mathcal{N} \leq 4$ Super Symmetry (SUSY) (including QCD), which in turn could be used to compute corresponding loop level amplitudes (see for a review [43]). It is important to note that all results mentioned above were almost impossible to obtain by standard textbook Feynman diagram methods.

The ideas and methods described above can be applied not only to the scattering amplitudes of on-shell states ( $S$-matrix elements) but to form factors of gauge invariant operators (local or none local) as well. The form factors of local gauge invariant operators are quite developed topics in the literature; see [44-52] and references therein. The general practice when studying form factors is to consider the case of local gauge invariant color singlet operators. 
However, one may also consider gauge invariant (the representation under global gauge transformation is not necessary singlet) nonlocal operators, for example, Wilson loops (lines) or their products [53-65]. An insertion of a Wilson line operator will then correspond to the off-shell or Reggeized gluon in such a formulation. These objects should be more familiar to the reader as gauge invariant offshell amplitudes [53-62] (also known as Reggeon amplitudes in the framework of Lipatov's effective Lagrangian), which appear within the context of $k_{T}$ or high-energy factorization [66-69] as well as in the study of processes at multi-Regge kinematics.

Up to the moment we already have scattering equation (connected prescription) representations for the form factors of operators from stress-tensor operator supermultiplet and scalar operators of the form $\operatorname{Tr}\left(\phi^{m}\right)$ [70,71]. Also the connected prescription formulas were extended to Standard Model amplitudes [72]. Besides, there are several results for the Grassmannian integral representation of form factors of operators from stress-tensor operator supermultiplet [73-76] and Wilson line operator insertions [63,64]; see also [65] for a recent interesting discussion of duality for Wilson loop form factors. ${ }^{1}$

The purpose of this work is to further pursue the string based approach to $\mathcal{N}=4$ super Yang-Mills and other fourdimensional gauge theories. Namely, we want to derive Grassmannian integral and scattering equation representation $([74,75]$ and $[63,64])$ of form factors and correlation functions of local and Wilson line operators in $\mathcal{N}=4$ SYM starting from four-dimensional ambitwistor string theory. Recently we have already provided such a derivation for the case of the Reggeon amplitude (Wilson line form factors) in [84]. This paper contains both extra details of the latter derivation together with its extension to the case of the form factors of local operators. In addition, we further investigate the relation between on-shell amplitudes and form factors and suggest a procedure that allows one to reconstruct (at tree and possible loop level) form factors and correlation functions of Wilson line operators starting directly from on-shell scattering amplitudes.

This paper is organized as follows: in Sec. II we introduce necessary definitions for the form factors of operators from stress-tensor supermultiplet and Wilson line operators.

In Sec. III, to make an article self-contained, we proceed with the recalling of general four-dimensional ambitwistor string theory formalism [11].

In Sec. IV we discuss our motivation to introduce the so-called gluing procedure-an operation, introduced for the first time in [84], that is given by convolution of the ambitwistor vertex operators with some function of

\footnotetext{
${ }^{1}$ A very close subject is the twistor and Lorentz harmonic chiral superspace formulation of form factors and correlation functions developed in [77-83].
}

external kinematical data. Based on this gluing procedure we present expressions for string theory generalized vertex operators (string theory composite operators) corresponding to the $\mathcal{N}=4$ SYM field theory stress-tensor operator as well as give some additional details regarding derivation of the results of [84]. Using these new string vertex operators we compute corresponding tree level string theory correlation functions and show that they correctly reproduce the results of previously obtained Grassmannian integral representations of stress tensor supermultiplet form factors [74] as well as form factors and correlation functions of Wilson line operators $[63,64]$.

Section V contains a detailed review based on [26] about the relation between Grassmannian integral and RSV (scattering equation) representations of the on-shell amplitudes, which play a very important role in our construction as well. At the end of the section we briefly discuss one simple self-consistency check of our construction.

In Sec. VI we further discuss the gluing procedure. We show that one can formally apply it (a convolution with some function of external kinematical data) directly on the level of on-shell amplitudes represented as the sum of BCFW terms (both at tree and, probably, integrand level). We formalize this by introducing the notion of gluing operator $\hat{A}$. Using the gluing operator we reproduce several previously obtained $[53,63,64]$ results for Wilson line form factors (Reggeon amplitudes) including the three-point correlation function of Reggeized gluons.

Finally, in Sec. VII we present our conclusion and discuss possible future research directions. Appendices A and $\mathrm{B}$ contain the computational details of the form factor gluing procedure.

\section{FORM FACTORS OF LOCAL AND WILSON LINE OPERATORS}

In this work we are interested in the ambitwistor string description of form factors of the Wilson line and local operators in $\mathcal{N}=4 \mathrm{SYM}$. $\mathcal{N}=4 \mathrm{SYM}$ is a maximally supersymmetric gauge theory in four space-time dimensions $[85,86]$. Its 16 on-shell states (their creation/ annihilation operators) could be conveniently described using $\mathcal{N}=4$ on-shell chiral superfield [87],

$$
\begin{aligned}
\Omega= & g^{+}+\tilde{\eta}_{A} \psi^{A}+\frac{1}{2 !} \tilde{\eta}_{A} \tilde{\eta}_{B} \phi^{A B}+\frac{1}{3 !} \tilde{\eta}_{A} \tilde{\eta}_{B} \tilde{\eta}_{C} \epsilon^{A B C D} \bar{\psi}_{D} \\
& +\frac{1}{4 !} \tilde{\eta}_{A} \tilde{\eta}_{B} \tilde{\eta}_{C} \tilde{\eta}_{D} \epsilon^{A B C D} g^{-} .
\end{aligned}
$$

Here, $g^{+}, g^{-}$denote creation/annihilation operators of gluons with +1 and -1 helicities, $\psi^{A}$ are creation/annihilation operators of four Weyl spinors with negative helicity $-1 / 2$, $\bar{\psi}_{A}$ are creation/annihilation operators of four Weyl spinors with positive helicity, and $\phi^{A B}$ stand for creation/annihilation operators of six scalars (antisymmetric in the $S U(4)_{R}$ $R$-symmetry group indices $A B$ ). All $\mathcal{N}=4 \mathrm{SYM}$ fields 
transform in the adjoint representation of the $S U\left(N_{c}\right)$ gauge group. In what follows we will also need superstates defined by the action of superfield creation/annihilation operators on a vacuum. For the $n$-particle superstate we have

$$
\left|\Omega_{1} \Omega_{2} \cdots \Omega_{n}\right\rangle \equiv \Omega_{1} \Omega_{2} \cdots \Omega_{n}|0\rangle .
$$

Form factors of Wilson line operators are generally used to describe gauge invariant off-shell or Reggeon amplitudes [53-62]. The Wilson line operators used to describe offshell Reggeized gluons are defined as [60]

$$
\begin{aligned}
\mathcal{W}_{p}^{c}(k)= & \int d^{4} x e^{i x \cdot k} \operatorname{Tr}\left\{\frac{1}{\pi g} t^{c} \mathcal{P} \exp \right. \\
& \left.\times\left[\frac{i g}{\sqrt{2}} \int_{-\infty}^{\infty} d s p \cdot A_{b}(x+s p) t^{b}\right]\right\},
\end{aligned}
$$

where $t^{c}$ is the $S U\left(N_{c}\right)$ generator $^{2}$ and we also used the so-called $k_{T}$ decomposition of the off-shell gluon momentum $k, k^{2} \neq 0$,

$$
k^{\mu}=x p^{\mu}+k_{T}^{\mu}
$$

Here, $p$ is the off-shell gluon direction (also known as the gluon polarization vector), such that $p^{2}=0, p \cdot k=0$, and $x \in[0,1]$. Such decomposition is generally parametrized by an auxiliary light-cone four-vector $q^{\mu}$, so that

$k_{T}^{\mu}(q)=k^{\mu}-x(q) p^{\mu} \quad$ with $\quad x(q)=\frac{q \cdot k}{q \cdot p} \quad$ and $\quad q^{2}=0$.

As momentum $k_{T}^{\mu}$ is transverse with respect to both $p^{\mu}$ and $q^{\mu}$ vectors, one can decompose it into the basis of two "polarization" vectors ${ }^{3}$ as [53]

$$
\begin{aligned}
k_{T}^{\mu}(q) & =-\frac{\kappa}{2} \frac{\left\langle p\left|\gamma^{\mu}\right| q\right]}{[p q]}-\frac{\kappa^{*}}{2} \frac{\left\langle q\left|\gamma^{\mu}\right| p\right]}{\langle q p\rangle} \quad \text { with } \quad \kappa=\frac{\langle q|\not k| p]}{\langle q p\rangle}, \\
\kappa^{*} & =\frac{\langle p|\not k| q]}{[p q]} .
\end{aligned}
$$

It is easy to see that $k^{2}=-\kappa \kappa^{*}$ and both $\kappa$ and $\kappa^{*}$ are independent of auxiliary four-vector $q^{\mu}$ [53]. Another useful relation, which is a direct consequence of $k_{T}$ decomposition and will be used often in practical calculations later on, is

$$
k|p\rangle=\mid p] \kappa^{*} .
$$

Note that the Wilson line operator we use to describe offshell gluon is colored. It is invariant $\delta \mathcal{W}_{p}^{c}(k)=0$ under

\footnotetext{
${ }^{2}$ The color generators are normalized as $\operatorname{Tr}\left(t^{a} t^{b}\right)=\delta^{a b}$.

${ }^{3}$ Here we used the helicity spinor decomposition of lightlike four-vectors $p$ and $q$.
}

local infinitesimal gauge transformations $\delta A_{\mu}=\left[D_{\mu}, \chi\right]$ with $\chi$ decreasing at $x \rightarrow \infty$. At the same time it transforms under global adjoint transformations of $S U\left(N_{c}\right)$ with constant $\chi$ as $[56,57]$

$$
\delta \mathcal{W}_{p}(k)=g\left[\mathcal{W}_{p}(k), \chi\right] .
$$

The form factor of Wilson line operator or gauge invariant amplitude with one off-shell and $n$ on-shell gluons is then given by [60]

$$
\mathcal{A}_{n+1}\left(1^{ \pm}, \ldots, n^{ \pm}, g_{n+1}^{*}\right)=\left\langle\left\{k_{i}, \epsilon_{i}, c_{i}\right\}_{i=1}^{n}\left|\mathcal{W}_{p}^{c_{n+1}}(k)\right| 0\right\rangle,
$$

where the asterisk denotes off-shell gluon, while $p, k$, and $c$ stand for its direction, momentum, and color index. Next $\left\langle\left\{k_{i}, \epsilon_{i}, c_{i}\right\}_{i=1}^{m}\right|=\bigotimes_{i=1}^{m}\left\langle k_{i}, \varepsilon_{i}, c_{i}\right|$ and $\left\langle k_{i}, \varepsilon_{i}, c_{i}\right|$ denote the on-shell gluon state with momentum $k_{i}$, polarization vector $\varepsilon_{i}^{-}$or $\varepsilon_{i}^{+}$, and color index $c_{i}$. Also in the case when there is no confusion in the position of the Wilson line operator insertion, the latter will be labeled just by $g^{*}$. Form factors with multiple Wilson line insertions or amplitudes with multiple off-shell gluons can be represented in a similar fashion,

$$
\begin{aligned}
& \mathcal{A}_{m+n}\left(1^{ \pm}, \ldots, m^{ \pm}, g_{m+1}^{*}, \ldots, g_{n+m}^{*}\right) \\
& \quad=\left\langle\left\{k_{i}, \epsilon_{i}, c_{i}\right\}_{i=1}^{m}\left|\prod_{j=1}^{n} \mathcal{W}_{p_{j+m}}^{c_{j+m}}\left(k_{j+m}\right)\right| 0\right\rangle,
\end{aligned}
$$

where $p_{i+m}$ is the direction of the $i$ th $(i=1, \ldots, n)$ off-shell gluon and $k_{i+m}$ is its off-shell momentum. As a function of kinematical variables this amplitude is written as

$$
\begin{aligned}
& \mathcal{A}_{m+n}\left(1^{ \pm}, \ldots, g_{n+m}^{*}\right) \\
& \quad=\mathcal{A}_{m+n}\left(\left\{\lambda_{i}, \tilde{\lambda}_{i}, \pm, c_{i}\right\}_{i=1}^{m} ;\left\{k_{j}, \lambda_{p, j}, \tilde{\lambda}_{p, j}, c_{j}\right\}_{j=m+1}^{m+n}\right),
\end{aligned}
$$

where $\lambda_{p, j}, \tilde{\lambda}_{p, j}$ are spinors coming from helicity spinor decomposition of polarization vector of $j$ th Reggeized gluon. In the case when only off-shell gluons are present (the correlation function of Wilson line operator insertions) we have

$$
\mathcal{A}_{0+n}\left(g_{1}^{*} \cdots g_{n}^{*}\right)=\left\langle 0\left|\mathcal{W}_{p_{1}}^{c_{1}}\left(k_{1}\right) \cdots \mathcal{W}_{p_{n}}^{c_{n}}\left(k_{n}\right)\right| 0\right\rangle .
$$

Of course, it is also possible to consider color ordered versions of Wilson line form factors, while the original offshell amplitudes (Wilson line form factors) are then recovered using color decomposition ${ }^{4}$ :

\footnotetext{
${ }^{4}$ See, for example, $[63,88]$.
} 


$$
\begin{aligned}
\mathcal{A}_{n+m}^{*} & \left(1^{ \pm}, \ldots, m^{ \pm}, g_{m+1}^{*}, \ldots, g_{n+m}^{*}\right) \\
= & g^{n-2} \sum_{\sigma \in S_{n+m} / Z_{n+m}} \operatorname{tr}\left(t^{a_{\sigma(1)}} \cdots t^{a_{\sigma(n+m)}}\right) \\
& \times A_{n+m}^{*}\left(\sigma\left(1^{ \pm}\right), \ldots, \sigma\left(g_{n+m}^{*}\right)\right) .
\end{aligned}
$$

Note that in the planar limit this decomposition is valid for both arbitrary tree and loop level amplitudes.

In the case of $\mathcal{N}=4$ SYM one may also consider other than gluons on-shell states from the $\mathcal{N}=4$ supermultiplet. The corresponding $\mathcal{N}=4$ SYM superamplitudes are then given by

$$
\begin{aligned}
& A_{m+n}^{*}\left(\Omega_{1}, \ldots, \Omega_{m}, g_{m+1}^{*}, \ldots, g_{n+m}^{*}\right) \\
& \quad=\left\langle\Omega_{1} \ldots \Omega_{m}\left|\prod_{j=1}^{n} \mathcal{W}_{p_{m+j}}\left(k_{m+j}\right)\right| 0\right\rangle,
\end{aligned}
$$

and the explicit dependence of $A_{m+n}^{*}\left(\Omega_{1}, \ldots, g_{m+n}^{*}\right)$ amplitude on kinematical variables takes the form

$$
\begin{aligned}
& A_{m+n}^{*}\left(\Omega_{1}, \ldots, g_{m+n}^{*}\right) \\
& \quad=A_{m+n}^{*}\left(\left\{\lambda_{i}, \tilde{\lambda}_{i}, \tilde{\eta}_{i}\right\}_{i=1}^{m} ;\left\{k_{j}, \lambda_{p, j}, \tilde{\lambda}_{p, j}\right\}_{j=m+1}^{m+n}\right) .
\end{aligned}
$$

The above superamplitude contains not only component amplitudes with on-shell gluons but also all amplitudes with other on-shell states from the $\mathcal{N}=4$ supermultiplet. The helicity spinors $\lambda_{i}, \tilde{\lambda}_{i}$ encode kinematics of on-shell states, while $\tilde{\eta}_{i}$ encodes their helicity content. Off-shell momentum $k_{i}$ and light-cone direction vector $p_{i}=\lambda_{p, i} \tilde{\lambda}_{p, i}$ encode information related to Wilson line operator insertion. So, in what follows we will be considering the partially supersymmetrized version of amplitudes (2.10) with on-shell states treated in a supersymmetric manner, while Wilson line operators ("off-shell states") are left unsupersymmetrized. The component amplitudes containing gluons, scalars, and fermions may then be extracted as coefficients in the $\tilde{\eta}$ expansion of the $A_{m+n}^{*}$ superamplitude similar to the case of ordinary on-shell amplitudes and super form factors.

While our present consideration should be applicable not only to the Wilson line but to arbitrary local operators, here for concreteness we will restrict ourselves to the case of operators from a stress-tensor operator supermultiplet. When considering the latter the general practice is to focus on the chiral part of this multiplet. Using the harmonic superspace approach $[89,90]$ it is given by [90-93]

$$
\mathcal{T}\left(x, \theta^{+}\right)=\operatorname{tr}\left(\phi^{++} \phi^{++}\right)+\cdots+\frac{1}{3}\left(\theta^{+}\right)^{4} \mathcal{L},
$$

where $u_{A}^{+a}, u_{A}^{-a^{\prime}}$ is a set of harmonic coordinates parametrizing coset $\frac{S U(4)}{S U(2) \times S U(2)^{\prime} \times U(1)}$ and $\theta_{\alpha}^{+a}=\theta_{\alpha}^{A} u_{A}^{+a}$,

\footnotetext{
${ }^{5}$ See corresponding discussion in the Conclusion.
}

$\theta_{\alpha}^{-a^{\prime}}=\theta_{\alpha}^{A} u_{A}^{-a^{\prime}}$. Here, $A$ is the $S U(4)_{R}$ index, $a$ and $a^{\prime}$ are $S U(2)$ indices, and \pm denote $U(1)$ charges. For example, $\epsilon^{a b} \phi^{++}=\phi^{A B} u_{A}^{+a} u_{A}^{+b}$, where $\phi^{A B}$ is the scalar field from the $\mathcal{N}=4$ Lagrangian. The color ordered form factors of operators from the chiral truncation of stresstensor operator supermultiplet $F_{n}$ are then given by

$$
\begin{aligned}
F_{n}\left(\Omega_{1}, \ldots, \Omega_{n} ; \mathcal{T}\right) & \equiv\left\langle\Omega_{1} \cdots \Omega_{n}\left|\mathcal{T}\left(q, \gamma^{-}\right)\right| 0\right\rangle \\
& =F_{n}\left(\left\{\lambda_{i}, \tilde{\lambda}_{i}, \tilde{\eta}_{i}\right\}_{i=1}^{n} ;\left\{q, \gamma^{-}\right\}\right),
\end{aligned}
$$

where $\left\{\lambda_{i}, \tilde{\lambda}_{i}, \tilde{\eta}_{i}\right\}_{i=1}^{n}$ are kinematical and helicity data of the on-shell states, $q$ is the operator momentum, and $\gamma^{-}$ parametrizes the operator content of the chiral part of $\mathcal{N}=4$ SYM stress-tensor operator supermultiplet. Here, we have also performed the Fourier transformation from variables $x, \theta^{+}$to $q, \gamma^{-}[91,92]$. The full physical form factor may then be restored from its color ordered version using standard color decomposition formula

$$
\begin{aligned}
\mathcal{F}_{n}\left(\Omega_{1}, \ldots, \Omega_{n} ; \mathcal{T}\right)= & g^{n-2} \sum_{\sigma \in S_{n} / Z_{n}} \operatorname{tr}\left(t^{a_{\sigma(1)}} \cdots t^{a_{\sigma(n)}}\right) \\
& \times F_{n}\left(\sigma\left(\Omega_{1}\right), \ldots, \sigma\left(\Omega_{n}\right) ; \mathcal{T}\right),
\end{aligned}
$$

where $S_{n} / Z_{n}$ denotes all noncyclic permutations of $n$ objects. As in the case of off-shell amplitudes, this formula is valid for both arbitrary tree and loop level form factors in the planar limit. ${ }^{6}$ At least at tree level the form factors of the full stress-tensor operator supermultiplet can be reconstructed if the explicit form of (2.17) is known [91].

\section{FOUR-DIMENSIONAL AMBITWISTOR STRINGS}

\section{A. General formalism}

As was already mentioned in the Introduction, to describe form factors of local and Wilson line operators we will be using the four-dimensional ambitwistor string theory originally formulated in [11]. Here for completeness we will discuss essential details of dimensional ambitwistor string theory. Our presentation of this theory here closely follows [11], and we refer the interested reader to this original paper and [94] for further details.

The target space of four-dimensional ambitwistor string is given by projective ambitwistor space $\mathbb{P A}$. The latter is the supersymmetrized space of complex null geodesics in a complexified Minkowski given by a quadric $Z \cdot W=0$ inside the product of twistor and dual twistor spaces $\mathbb{P} \mathbb{T} \times \mathbb{P}^{*}$ quotient by relative scaling $Z \cdot \partial_{Z}-W \cdot \partial_{W}$ :

$$
\mathbb{P A}=\left\{(Z, W) \in \mathbb{T} \times \mathbb{T}^{*} \mid Z \cdot W=0\right\} /\left\{Z \cdot \partial_{Z}-W \cdot \partial_{W}\right\} .
$$

\footnotetext{
${ }^{6}$ At loop level one should take into account appropriate powers of t'Hooft coupling constant $g^{2} N_{c}$, which were suppressed here.
} 
In the case of $\mathcal{N}$ supersymmetries $Z=\left(\lambda_{\alpha}, \mu^{\dot{\alpha}}, \chi^{r}\right) \in$ $\mathbb{T}=\mathbb{C}^{4 \mid \mathcal{N}}, \quad W=(\tilde{\mu}, \tilde{\lambda}, \tilde{\chi}) \in \mathbb{T}^{*}, \quad$ and $\quad Z \cdot W=\lambda_{\alpha} \tilde{\mu}^{\alpha}+$ $\mu^{\dot{\alpha}} \tilde{\lambda}_{\dot{\alpha}}+\chi^{r} \tilde{\chi}_{r}$, where $\chi, \tilde{\chi}$ are fermionic, $\alpha=0,1, \dot{\alpha}=\dot{0}$, $\mathrm{i}$, and $r=1, \ldots, \mathcal{N}$ is the $R$-symmetry index. The point $(x, \theta, \tilde{\theta})$ in nonchiral super Minkowski space corresponds to a quardric $\mathbb{C} \mathbb{P}^{1} \times \mathbb{C P}^{1}$ parametrized by $(\lambda, \tilde{\lambda})$ spinors. The correspondence is realized by the standard twistor incidence relations

$$
\begin{array}{ll}
\mu^{\dot{\alpha}}=i\left(x^{\alpha \dot{\alpha}}+i \theta^{r \alpha} \tilde{\theta}_{r}^{\dot{\alpha}}\right) \lambda_{\alpha}, & \chi^{r}=\theta^{r \alpha} \lambda_{\alpha}, \\
\tilde{\mu}^{\alpha}=-i\left(x^{\alpha \dot{\alpha}}-i \theta^{r \alpha} \tilde{\theta}_{r}^{\dot{\alpha}}\right) \tilde{\lambda}_{\dot{\alpha}}, & \tilde{\chi}_{r}=\tilde{\theta}_{r}^{\dot{\alpha}} \tilde{\lambda}_{\dot{\alpha}} .
\end{array}
$$

It is easy to check that this quardric lies in $Z \cdot W=0$.

The four-dimensional ambitwistor string consists from world-sheet spinors $(Z, W)$ with values in $\mathbb{T} \times \mathbb{T}^{*}$ and $\operatorname{GL}(1, \mathbb{C})$ gauge field $a$ acting as a Lagrange multiplier for the constraint $Z \cdot W=0$. In the conformal gauge the action is given by ${ }^{7}$

$$
S=\frac{1}{2 \pi} \int_{\Sigma} W \cdot \bar{\partial} Z-Z \cdot \bar{\partial} W+a Z \cdot W+S_{J},
$$

where $\bar{\partial}=\mathrm{d} \bar{\sigma} \partial_{\bar{\sigma}}(\sigma, \bar{\sigma}$ are some local holomorphic and antiholomorphic coordinates on Riemann surface $\Sigma$ ) and $S_{J}$ is the action for a world-sheet Kac-Moody current algebra $J \in \Omega^{0}\left(\Sigma, K_{\Sigma} \otimes \mathfrak{g}\right)$ for some Lie algebra $\mathfrak{g}$. Here $K_{\Sigma}$ denotes the canonical bundle on surface $\Sigma$ and the remaining world-sheet fields take values in

$$
\begin{gathered}
Z \in \Omega^{0}\left(\Sigma, K_{\Sigma}^{1 / 2} \otimes \mathbb{T}\right), \\
W \in \Omega^{0}\left(\Sigma, K_{\Sigma}^{1 / 2} \otimes \mathbb{T}^{*}\right), \\
a \in \Omega^{0,1}(\Sigma),
\end{gathered}
$$

where powers of the canonical bundle denote field conformal weights. The above action is invariant under a gauge symmetry,

$$
Z^{I} \rightarrow e^{\gamma} Z^{I}, \quad W_{I} \rightarrow e^{-\gamma} W_{I}, \quad a \rightarrow a-2 \bar{\partial} \gamma,
$$

that separates the target space into equivalence classes with respect to the action of $Z \cdot \partial_{Z}-W \cdot \partial_{W}$. The gauge fixing of world-sheet diffeomorphism symmetry ${ }^{8}$ and the above gauge redundancy via standard Becchi-Rouet-Stora-Tyutin (BRST) procedure leads to the introduction of the standard

\footnotetext{
${ }^{7}$ It is obtained by chiral pullback of contact structure on ambitwistor space $\Theta=\frac{i}{2}(Z \cdot \mathrm{d} W-W \cdot \mathrm{d} Z)$ [11]. Note that similar action first appeared in [2] in the context of open twistor string theory.

${ }^{8}$ In a general gauge, the $\bar{\partial}$ operator in (3.4) is replaced by operator $\bar{\partial}_{\tilde{e}}=\bar{\partial}+\tilde{e} \partial$ parametrizing the world-sheet diffeomorphism freedom.
}

reparametrization (Virasoro) $(b, c)$ together with GL(1) $(u, v)$ ghost systems:

$$
\begin{array}{r}
c \in \Pi \Omega^{0}\left(\Sigma, T_{\Sigma}\right), \quad v \in \Pi \Omega^{0}(\Sigma), \\
b \in \Pi \Omega^{0}\left(\Sigma, K_{\Sigma}^{2}\right), \quad u \in \Pi \Omega^{0}\left(\Sigma, K_{\Sigma}\right),
\end{array}
$$

where $T_{\Sigma}$ denotes the tangent bundle on surface $\Sigma$ and $\Pi \Omega^{0}(\Sigma, E)$ denotes the space of fermion-valued sections of $E$. The full world-sheet action is then given by

$S=\frac{1}{2 \pi} \int_{\Sigma} W \cdot \bar{\partial} Z-Z \cdot \bar{\partial} W+b \bar{\partial} c+u \bar{\partial} v+S_{J}$,

and the BRST operator takes the form

$$
Q=\oint c T+v Z \cdot W+Q_{\mathrm{gh}},
$$

where $T=W \cdot \partial Z-Z \cdot \partial W+T_{J}$ is the world-sheet stress-energy tensor.

\section{B. String vertex operators and their correlation functions}

To calculate string scattering amplitudes we need vertex operators. In general they are given by first-quantized wave functions of external states translated into world-sheet operator insertions. Penrose transform allows us to relate solutions to massless field equations in Minkowski space to cohomology classes on projective twistor space. In the case of Yang-Mills theory ambitwistor string vertex operators are obtained by pairing pullbacks of general ambitwistor space wave functions $\alpha \in H^{1}(\mathbb{P A}, \mathcal{O})[\bar{\partial}$-closed worldsheet $(0,1)$ forms] with Kac-Moody currents $J \cdot T_{a}$ to get $\mathcal{V}_{a}=\int_{\Sigma} \alpha J \cdot T_{a}$. For two types of momentum eigenstates (pullbacks from either twistor or dual twistor space) we get [11]

$$
\begin{aligned}
& \mathcal{V}_{a}=\int \frac{\mathrm{d} s_{a}}{s_{a}} \bar{\delta}^{2}\left(\lambda_{a}-s_{a} \lambda\right) \mathrm{e}^{i s_{a}\left(\left[\mu \tilde{\lambda}_{a}\right]+\chi^{r} \tilde{\eta}_{a r}\right)} J \cdot T_{a}, \\
& \tilde{\mathcal{V}}_{a}^{\prime}=\int \frac{\mathrm{d} s_{a}}{s_{a}} \bar{\delta}^{2}\left(\tilde{\lambda}_{a}-s_{a} \tilde{\lambda}\right) \mathrm{e}^{i s_{a}\left(\left\langle\tilde{\mu} \lambda_{a}\right\rangle+\tilde{\chi}_{r} \eta_{a}^{r}\right)} J \cdot T_{a},
\end{aligned}
$$

where $\bar{\delta}(z)=\bar{\partial}(1 / 2 \pi i z)$ for complex $z$. Note that these vertex operators are $Q$ closed $^{9}$ and satisfy $\left\{Q, \mathcal{V}_{a}\right\}=$ $\left\{Q, \tilde{\mathcal{V}}_{a}^{\prime}\right\}=0$. To facilitate further comparison with Grassmannian integral representation, it is convenient to introduce a slightly different representation for the second

\footnotetext{
${ }^{9}$ It should be noted that in general this theory is anomalous and has nonzero central charge, so that $Q^{2} \neq 0[11,94]$, but one can adjust the central charge of Kac-Moody current algebra to get $Q^{2}=0$ at least for a lower genus Rieman surface [10].
} 
vertex operator. It is obtained by a Fourier transform ${ }^{10}$ of the $\eta$ 's into $\tilde{\eta}$ 's:

$$
\tilde{\mathcal{V}}_{a}=\int \frac{\mathrm{d} s_{a}}{s_{a}} \bar{\delta}^{2 \mid \mathcal{N}}\left(\tilde{\lambda}_{a}-s_{a} \tilde{\lambda} \mid \tilde{\eta}_{a}-s_{a} \tilde{\chi}\right) \mathrm{e}^{i s_{a}\left\langle\tilde{\mu} \lambda_{a}\right\rangle} J \cdot T_{a} .
$$

In the case of $\mathcal{N}=3$ these vertex operators together encode all 16 degrees of freedom of $\mathcal{N}=4$ SYM theory. For $\mathcal{N}=4$, on the other hand, each of them contains all $\mathcal{N}=4$ SYM on-shell states. In our consideration of $\mathcal{N}=4$ SYM to obtain maximally supersymmetric superamplitudes we will use the second option and these vertex operators interchangeably.

$\mathrm{N}^{k-2}$ MHV superamplitudes may then be obtained, for example, as correlation functions of $k$ operators from dual twistor space and $n-k$ operators from twistor space [11] (here and below we omit color structures and already work with color ordered objects):

$$
A_{k, n}=\left\langle\tilde{\mathcal{V}}_{1} \cdots \tilde{\mathcal{V}}_{k} \mathcal{V}_{k+1} \cdots \mathcal{V}_{n}\right\rangle
$$

Instead of computing the infinite number of contractions required by exponentials in vertex operators, it is convenient to take exponentials into the action as sources,

$$
\int_{\Sigma} \sum_{i=1}^{k} i s_{i}\left\langle\tilde{\mu} \lambda_{i}\right\rangle \bar{\delta}\left(\sigma-\sigma_{i}\right)+\sum_{p=k+1}^{n} i s_{p}\left(\left[\mu \tilde{\lambda}_{p}\right]+\chi \tilde{\eta}_{p}\right) \bar{\delta}\left(\sigma-\sigma_{p}\right) .
$$

The corresponding equations of motion from this new action are then given by

$$
\begin{gathered}
\bar{\partial}_{\sigma} Z=\bar{\partial}(\lambda, \mu, \chi)=\sum_{i=1}^{k} s_{i}\left(\lambda_{i}, 0,0\right) \bar{\delta}\left(\sigma-\sigma_{i}\right), \\
\bar{\partial}_{\sigma} W=\bar{\partial}(\tilde{\mu}, \tilde{\lambda}, \tilde{\chi})=\sum_{p=k+1}^{n} s_{p}\left(0, \tilde{\lambda}_{p}, \tilde{\eta}_{p}\right) \bar{\delta}\left(\sigma-\sigma_{p}\right) .
\end{gathered}
$$

As $(Z, W)$ fields are world-sheet spinors the solutions to the above equations are unique ${ }^{11}$ and given by

$$
\begin{gathered}
Z(\sigma)=(\lambda, \mu, \chi)=\sum_{i=1}^{k} \frac{s_{i}\left(\lambda_{i}, 0,0\right)}{\sigma-\sigma_{i}}, \\
W(\sigma)=(\tilde{\mu}, \tilde{\lambda}, \tilde{\chi})=\sum_{p=k+1}^{n} \frac{s_{p}\left(0, \tilde{\lambda}_{p}, \tilde{\eta}_{p}\right)}{\sigma-\sigma_{p}} .
\end{gathered}
$$

Then the path integrals over $(Z, W)$ fields localize on the solutions (3.19) and (3.20), while the current correlator contributes the Parke-Taylor factor and for the color ordered on-shell amplitude we get [11]

\footnotetext{
${ }^{10}$ Note that in [11] instead a Fourier transform for the first operator from $\eta$ 's to $\tilde{\eta}$ 's was performed. The Grassmann part of the delta function is defined as usual: $\delta^{0 \mid \mathcal{N}}(\tilde{\eta})=\prod_{r=1}^{\mathcal{N}} \tilde{\eta}_{r}$.

${ }^{11}$ There no fermion zero modes on sphere.
}

$$
\begin{aligned}
A_{n, k}= & \int \frac{1}{\operatorname{VolGL}(2, \mathbb{C})} \prod_{a=1}^{n} \frac{\mathrm{d} s_{a} \mathrm{~d} \sigma_{a}}{s_{a}\left(\sigma_{a}-\sigma_{a+1}\right)} \\
& \times \prod_{p=k+1}^{n} \bar{\delta}^{2}\left(\lambda_{p}-s_{p} \lambda\left(\sigma_{p}\right)\right) \\
& \times \prod_{i=1}^{k} \bar{\delta}^{2 \mid \mathcal{N}}\left(\tilde{\lambda}_{i}-s_{i} \tilde{\lambda}\left(\sigma_{i}\right), \tilde{\eta}_{i}-s_{i} \tilde{\chi}\left(\sigma_{i}\right)\right) .
\end{aligned}
$$

Note that ghosts $c$ and $v$ develop ${ }^{12} n_{c}=3$ (number of conformal Killing vectors on sphere) and $n_{v}=1$ zero modes correspondingly, which result in the $\operatorname{GL}(2, \mathbb{C})$ quotient above. In terms of homogeneous coordinates on Riemann sphere $\sigma_{\alpha}=\frac{1}{s}(1, \sigma)$ the $W$ and $Z$ fields rescaled by a factor of $1 / s$ could be written as

$Z(\sigma)=\sum_{i=1}^{k} \frac{\left(\lambda_{i}, 0,0\right)}{\left(\sigma \sigma_{i}\right)}, \quad W(\sigma)=\sum_{p=k+1}^{n} \frac{\left(0, \tilde{\lambda}_{p}, \tilde{\eta}_{p}\right)}{\left(\sigma \sigma_{p}\right)}$,

where $(i j)=\sigma_{i \alpha} \sigma_{j}^{\alpha}$. Then the final formula for the above amplitude takes the form [11]

$$
\begin{aligned}
A_{n, k}= & \int \frac{1}{\operatorname{VolGL}(2, \mathbb{C})} \prod_{a=1}^{n} \frac{\mathrm{d}^{2} \sigma_{a}}{(a a+1)} \prod_{p=k+1}^{n} \bar{\delta}^{2}\left(\lambda_{p}-\lambda\left(\sigma_{p}\right)\right) \\
& \times \prod_{i=1}^{k} \bar{\delta}^{2 \mid \mathcal{N}}\left(\tilde{\lambda}_{i}-\tilde{\lambda}\left(\sigma_{i}\right), \tilde{\eta}_{i}-\tilde{\chi}\left(\sigma_{i}\right)\right) .
\end{aligned}
$$

The scattering equations then follow from the support of the delta functions,

$k_{a} \cdot P\left(\sigma_{a}\right)=\lambda_{a}^{\alpha} \tilde{\lambda}_{a}^{\dot{\alpha}} P_{\alpha \dot{\alpha}}\left(\sigma_{a}\right)=\lambda_{a}^{\alpha} \tilde{\lambda}_{a}^{\dot{\alpha}} \lambda_{\alpha}\left(\sigma_{a}\right) \tilde{\lambda}_{\dot{\alpha}}\left(\sigma_{a}\right)=0$.

It is important to note that the exact form of scattering equations themselves and scattering equation representations for amplitudes depends on which particle vertex operators were taken as $\tilde{\mathcal{V}}_{i}$ and which as $\mathcal{V}_{i}$ in (3.16). So, we have several equivalent representations for $A_{k, n}$. Their existence, as we will see in Sec. V, is related to the $G L(k)$ "gauge invariance" of Grassmannian integral representation for scattering amplitudes.

\section{GENERALIZED VERTEX OPERATORS}

In the previous section we saw the explicit form of the world-sheet vertex operators that corresponds to the onshell states of the $\mathcal{N}=4$ SYM field theory. In this section we will suggest construction of composite world-sheet operators in the ambitwistor string theory that will correspond to local and nonlocal gauge invariant operators in $\mathcal{N}=4$ SYM.

\footnotetext{
${ }^{12}$ This is easy to see with the help of the Riemann-Roch theorem recalling that $\operatorname{deg} T_{\Sigma}=-\operatorname{deg} K_{\Sigma}=2 g-2$, where $g$ is the genus of the Riemann surface.
} 
In [70] it was conjectured that it may be possible to obtain such operators considering appropriate terms in operator product expantion (OPE) of standard vertex operators (namely, OPE for Kac-Moody currents). Also ideologically similar attempts to construct generalization of vertex operators that should describe offshell states was taken in $[95,96]$ in the context of bosonic string theory. We, however, found such OPE based approaches unfitting for our purpose, though we do not claim that one cannot eventually succeed considering this direction.

Instead we want to leave the world-sheet structure of operators intact and consider external kinematics as only adjustable parameters. Namely, as our new generalized vertex operators $\mathcal{V}^{\text {gen }}$.. we want to consider the convolution of products of vertex operators $\mathcal{V}_{i}$ (3.13) with "target space wave functions" $\psi\left(\left\{\lambda_{i}, \tilde{\lambda}_{i}, \eta_{i}\right\}, \ldots\right)$-some rational functions on ambitwistor space. Here by "convolution" we mean the integration with respect to components of on-shell momenta $p_{i}=\lambda_{i} \tilde{\lambda}_{i}$ on which vertex operators depend ${ }^{13}$

$\mathcal{V}^{\text {gen }}=\int \psi\left(\left\{\lambda_{i}, \tilde{\lambda}_{i}, \eta_{i}\right\}, \ldots\right) \prod_{i} \mathcal{V}_{i} \frac{\mathrm{d}^{2} \lambda_{i} \mathrm{~d}^{2} \tilde{\lambda}_{i}}{\operatorname{Vol}[\operatorname{GL}(1)]} \mathrm{d}^{4} \tilde{\eta}_{i}$

Here we will understand integration with respect to $\mathrm{d}^{2} \lambda_{i} \mathrm{~d}^{2} \tilde{\lambda}_{i}$ as multidimensional contour integrals which in turn will be evaluated by residues. We will sometimes refer to this convolution as gluing operation. The ... in $\psi$ corresponds to possible dependence on parameters other than on-shell supermomenta $\lambda_{i}, \tilde{\lambda}_{i}, \eta_{i}$. To describe local and nonlocal operators in $\mathcal{N}=4$ SYM field theory target space wave functions $\psi$ should carry appropriate quantum numbers, so it is natural to take them in the form proportional to minimal form factors - tree level form factors of the corresponding operator with the minimal possible number of on-shell states. Such objects can be found from general symmetry arguments in the same lines as $\mathrm{MHV}_{3}$ and $\overline{\mathrm{MHV}}_{3}$ amplitudes [97] or simply by explicit evaluation. ${ }^{14}$

Another heuristic argument in favor of such a construction is the following: to obtain a vertex operator in ordinary string theory we usually consider a product of an appropriate polarization vector with a combination of world-sheet fields. For example, in the case of bosonic string theory the vertex operator $V_{\text {gr }}$ describing the graviton state is given by

\footnotetext{
${ }^{13}$ The conjectured vertex operator should also belong to BRST cohomology because the action of the $Q$ operator trivially commutes with the convolution considered here and the action of $Q$ on $\mathcal{V}^{\text {gen }}$.. reduces to the action of $Q$ on individual $\mathcal{V}_{i}$.

${ }^{14}$ Of course, it would be highly desirable to obtain some universal geometrical description of such target space wave functions. The possible direction to obtain such a description is to consider an appropriate polynomial solution of the classical (self-dual) equation of motion [98].
}

$$
V_{\mathrm{gr}}=\epsilon_{ \pm}^{\mu \nu} V_{\mu \nu}, \quad V_{\mu \nu}=\sqrt{g} g^{a b} \partial_{a} X_{\mu} \partial_{b} X_{\nu} \exp \left(i p^{\rho} X_{\rho}\right),
$$

where $X^{\mu}$ are world-sheet fields, $p^{\rho}$ are on-shell momenta of graviton, and $\epsilon_{ \pm}^{\mu \nu}$ is the polarization vector of graviton corresponding to momenta $p^{\rho}$. In suggested construction the target space wave functions $\psi$ is some kind of generalization of the polarization vector of the corresponding state, but instead of an ordinary scalar product we have integrations with respect to on-shell degrees of freedom we want to eliminate and instead of local world-sheet operator $V_{\mu \nu}$ we are considering multilocal operators $\prod_{i} \mathcal{V}_{i}$.

The suggested construction in principle should describe any gauge invariant operator in $\mathcal{N}=4$ SYM field theory. Initially it was successfully tested for the Wilson line operators (Reggeon state creation/annihilation operators) [84]. Here we want to give more details about derivation of this result as well as consider another new simple but important example of application of our construction. Namely, we want to suggest world-sheet generalized vertex operators that should describe the $\mathcal{N}=4$ SYM field theory stress-tensor supermultiplet.

\section{A. Generalized world-sheet vertex operator for $\mathcal{N}=4$ SYM field theory Wilson line operator}

Following the conjectures presented above for construction of generalized vertex operator $\mathcal{V}^{\mathrm{WL}}$ which should describe the field theory Wilson line operator, we have to choose target space wave functions $\psi$ proportional to the minimal Wilson line $A_{2,2+1}^{*}$. Namely, we will consider

$$
\begin{aligned}
\psi\left(\left\{\lambda_{j}, \tilde{\lambda}_{j}, \eta_{j}\right\}_{j=i}^{i+1},\left\{k, \lambda_{p}, \tilde{\lambda}_{p}\right\}\right)= & A_{2,2+1}^{*}\left(g^{*}, \Omega_{i}, \Omega_{i+1}\right) \\
& \times \text { color projectors. }
\end{aligned}
$$

This choice of $\psi$ was first considered in [84]. Here, as was mentioned before, and in Appendix A we will give a more detailed derivation of the results of [84].

So proceeding this way the ambitwistor string generalized vertex operator $\mathcal{V}^{\mathrm{WL}}$ for field theory Wilson line operator insertion could be written as

$$
\begin{aligned}
\mathcal{V}_{i, i+1}^{\mathrm{WL}}= & \left.\int \prod_{j=i}^{i+1} \frac{\mathrm{d}^{2} \lambda_{j} \mathrm{~d}^{2} \tilde{\lambda}_{j}}{\operatorname{Vol}[\operatorname{GL}(1)]} \mathrm{d}^{4} \tilde{\eta}_{j} A_{2,2+1}^{*}\left(g^{*}, \Omega_{i}, \Omega_{i+1}\right)\right|_{\lambda \rightarrow-\lambda} \\
& \times\left.\mathcal{V}_{i} \mathcal{V}_{i+1}\right|_{T^{a} T^{b} \rightarrow i f^{a b c} T^{c} \rightarrow T^{c}}
\end{aligned}
$$

where the vertex is supposed to be inserted at points $\sigma_{i}$, $\sigma_{i+1}, c$ is the color index of off-shell gluon, and we have used projection of the tensor product of two adjoint on-shell gluon color representations onto off-shell gluon adjoint color representation. Also because the on-shell state contents of $\mathcal{V}_{i}$ and $\tilde{\mathcal{V}}_{i}$ are identical, we actually consider combinations $\mathcal{V}_{i} \mathcal{V}_{i+1}, \mathcal{V}_{i} \tilde{\mathcal{V}}_{i+1}$, and $\tilde{\mathcal{V}}_{i} \tilde{\mathcal{V}}_{i+1}$ in the definition of $\mathcal{V}_{i, i+1}^{\mathrm{WL}}$ on equal footing. The minimal off-shell amplitude $A_{2,2+1}^{*}\left(g^{*}, \Omega_{i}, \Omega_{i+1}\right)$ is given by [63] 


$$
\begin{aligned}
A_{2,2+1}^{*}\left(g^{*}, \Omega_{i}, \Omega_{i+1}\right) & =\frac{1}{\kappa^{*}} \prod_{A=1}^{4} \frac{\partial}{\partial \tilde{\eta}_{p}^{A}}\left[\frac{\delta^{4}\left(k+\lambda_{i} \tilde{\lambda}_{i}+\lambda_{i+1} \tilde{\lambda}_{i+1}\right) \delta^{8}\left(\lambda_{p} \tilde{\eta}_{p}+\lambda_{i} \tilde{\eta}_{i}+\lambda_{i+1} \tilde{\eta}_{i+1}\right)}{\langle p i\rangle\langle i i+1\rangle\langle i+1 p\rangle}\right] \\
& =\frac{\delta^{4}\left(k+\lambda_{i} \tilde{\lambda}_{i}+\lambda_{i+1} \tilde{\lambda}_{i+1}\right)}{\kappa^{*}} \frac{\delta^{4}\left(\tilde{\eta}_{i}\langle p i+1\rangle+\tilde{\eta}_{i+1}\langle p i\rangle\right)}{\langle p i\rangle\langle i i+1\rangle\langle i+1 p\rangle} .
\end{aligned}
$$

Here $p$ is the off-shell gluon direction and $\kappa^{*}$ was defined in Sec. II when introducing $k_{T}$ decomposition of the off-shell gluon momentum $k$. It should be noted that each of the $\mathcal{V}$ operators above could be exchanged for the $\tilde{\mathcal{V}}$ operator, so that this vertex operator representation is not unique. Note also that the ambitwistor string vertex operator we got is nonlocal, which may be related to the fact that the Wilson line is a nonlocal object itself. The integrations over helicity spinors $\lambda_{i}, \tilde{\lambda}_{i}$ can be performed explicitly. The details of this computation can be found in Appendix A; see also [74].
After integrations we get (here and below we always assume the action of the projection operator $\partial_{\tilde{\eta}_{p}}^{4}$ acting on $\mathcal{V}_{i, i+1}^{\mathrm{WL}}$ and all correlation functions containing it)

$$
\mathcal{V}_{i, i+1}^{\mathrm{WL}}=\left.\frac{\langle\xi p\rangle}{\kappa^{*}} \int \frac{\mathrm{d} \beta_{2}}{\beta_{2}} \int \frac{\mathrm{d} \beta_{1}}{\beta_{1}} \frac{1}{\beta_{1}^{2} \beta_{2}} \mathcal{V}_{i} \mathcal{V}_{i+1}\right|_{T^{a} T^{b} \rightarrow i f^{a b c} T^{c} \rightarrow T^{c}}
$$

where

$$
\begin{gathered}
\lambda_{i}=\underline{\underline{\lambda}} i+\beta_{2} \underline{\underline{\lambda}}_{i+1}, \quad \tilde{\lambda}_{i}=\beta_{1} \underline{\underline{\lambda}}_{i}+\frac{\left(1+\beta_{1}\right)}{\beta_{2}} \underline{\tilde{\lambda}}_{i+1}, \quad \tilde{\eta}_{i}=-\beta_{1} \underline{\underline{\underline{\eta}}}_{i}, \\
\lambda_{i+1}=\underline{\underline{\lambda}}_{i+1}+\frac{\left(1+\beta_{1}\right)}{\beta_{1} \beta_{2}} \underline{\lambda}_{i}, \quad
\end{gathered}
$$

with

$$
\underline{\lambda}_{i}=\lambda_{p}, \underline{\tilde{\lambda}}_{i}=\frac{\langle\xi| k}{\langle\xi p\rangle}, \quad \underline{\underline{\tilde{\eta}}}_{i}=\tilde{\eta}_{p} ; \quad \underline{\lambda}_{i+1}=\lambda_{\xi}, \quad \underline{\tilde{\lambda}}_{i+1}=\frac{\langle p| k}{\langle\xi p\rangle}, \quad \underline{\tilde{\eta}}_{i+1}=0
$$

where $\lambda_{\xi} \equiv\langle\xi|$ is some arbitrary spinor. It is useful to identify it with the spinor $\lambda_{q}$ coming from helicity spinor decomposition of auxiliary vector $q$ arising in $k_{T}$ decomposition of off-shell gluon momentum $k$.

The off-shell amplitude with one off-shell and $n$ on-shell legs is then given by the following ambitwistor string correlation function (let us remind the reader that we are considering an already color ordered object):

$$
A_{k, n+1}^{*}=\left\langle\tilde{\mathcal{V}}_{1} \cdots \tilde{\mathcal{V}}_{k} \mathcal{V}_{k+1} \cdots \mathcal{V}_{n} \mathcal{V}_{n+1, n+2}^{\mathrm{WL}}\right\rangle
$$

Evaluating the first ambitwistor string correlator of on-shell vertexes with the help of (3.21) we get

$$
\begin{aligned}
A_{k, n+1}^{*}= & \frac{\langle\xi p\rangle}{\kappa^{*}} \int \frac{\mathrm{d} \beta_{2}}{\beta_{2}} \int \frac{\mathrm{d} \beta_{1}}{\beta_{1}} \frac{1}{\beta_{1}^{2} \beta_{2}} \frac{1}{\operatorname{VolGL}(2, \mathbb{C})} \\
& \times \int \prod_{a=1}^{n+2} \frac{\mathrm{d} s_{a} \mathrm{~d} \sigma_{a}}{s_{a}\left(\sigma_{a}-\sigma_{a+1}\right)} \prod_{p=k+1}^{n+2} \bar{\delta}^{2}\left(\lambda_{p}-s_{p} \lambda\left(\sigma_{p}\right)\right) \prod_{i=1}^{k} \bar{\delta}^{2 \mid 4}\left(\tilde{\lambda}_{i}-s_{i} \tilde{\lambda}\left(\sigma_{i}\right), \tilde{\eta}_{i}-s_{i} \tilde{\chi}\left(\sigma_{i}\right)\right) .
\end{aligned}
$$

We want to remind the reader that in this formula kinematical variables $\left\{\lambda_{i}, \tilde{\lambda}_{i}, \eta_{i}\right\}_{i=n+1}^{n+2}$ depend on $\beta_{1}$ and $\beta_{2}$ according to (4.7)-(4.9).

Next we want to perform integrations with respect to $\beta_{1}$ and $\beta_{2}$ to obtain formulas similar to (3.23). It is rather complicated to perform integrations over $\beta_{1}$ and $\beta_{2}$ in the expression above due to the nonlinear structure of delta function arguments with respect to $\beta_{1}$ and $\beta_{2}$. So we want to linearize them first. For this purpose we are introducing unity decomposition in the form [26]

$$
\begin{aligned}
1= & \frac{1}{\operatorname{VolGL}(k)} \int \mathrm{d}^{k \times(n+2)} C \mathrm{~d}^{k \times k} L(\operatorname{det} L)^{n+2} \delta^{k \times(n+2)} \\
& \times\left(C-L \cdot C^{V}[s, \sigma]\right),
\end{aligned}
$$


where the integral over the $L$ matrix is an integral over $\operatorname{GL}(k)$ linear transformations and $C^{V}[\sigma]$ denotes the Veronese map from $\left(\mathbb{C}^{2}\right)^{n+2} / \mathrm{GL}(2)$ to $G r(k, n+2)$ Grassmannian [26] (see also [70]),

$$
C^{V}[s, \sigma]=\left(\begin{array}{cccc}
\vdots & \vdots & \cdots & \vdots \\
\sigma^{V}\left[s_{1}, \sigma_{1}\right] & \sigma^{V}\left[s_{2}, \sigma_{2}\right] & \cdots & \sigma^{V}\left[s_{n+2}, \sigma_{n+2}\right] \\
\vdots & \vdots & \cdots & \vdots
\end{array}\right), \sigma^{V}[s, \sigma] \equiv\left(\begin{array}{c}
\xi \\
\xi \sigma \\
\vdots \\
\xi \sigma^{k-1}
\end{array}\right)
$$

where $[4,70]$

$$
\begin{gathered}
\xi_{i}=s_{i}^{-1} \prod_{j=1, j \neq i}^{k}\left(\sigma_{j}-\sigma_{i}\right)^{-1}, \quad i \in(1, k), \\
\xi_{i}=s_{i} \prod_{j=1}^{k}\left(\sigma_{j}-\sigma_{i}\right)^{-1}, \quad i \in(k+1, n+2) .
\end{gathered}
$$

Rearranging (4.11) we can write it as

$$
\begin{aligned}
A_{k, n+1}^{*}= & \frac{\langle\xi p\rangle}{\kappa^{*}} \int \frac{\mathrm{d} \beta_{2}}{\beta_{2}} \int \frac{\mathrm{d} \beta_{1}}{\beta_{1}} \frac{1}{\beta_{1}^{2} \beta_{2}} \frac{1}{\operatorname{VolGL}(k)} \\
& \times \int \mathrm{d}^{k \times(n+2)} C F(C) \delta^{k \times 2}(C \cdot \tilde{\lambda}) \delta^{k \times 4}(C \cdot \tilde{\eta}) \delta^{(n+2-k) \times 2}\left(C^{\perp} \cdot \lambda\right),
\end{aligned}
$$

where

$$
F(C)=\int \frac{1}{\operatorname{VolGL}(2, \mathbb{C})} \prod_{a=1}^{n+2} \frac{\mathrm{d} s_{a} \mathrm{~d} \sigma_{a}}{s_{a}\left(\sigma_{a}-\sigma_{a+1}\right)} \mathrm{d}^{k \times k} L \delta^{k \times(n+2)}\left(C-L \cdot C^{V}[s, \sigma]\right),
$$

and

$$
\begin{aligned}
\delta^{k \times 2}(C \cdot \tilde{\lambda}) & \equiv \prod_{a=1}^{k} \delta^{2}\left(\sum_{i=1}^{n} c_{a i} \tilde{\lambda}_{i}\right), \quad \delta^{(n+2-k) \times 2}\left(C^{\perp} \cdot \lambda\right) \equiv \prod_{b=k+1}^{n+2} \delta^{2}\left(\sum_{j=1}^{n+2} c_{b j}^{\perp} \lambda_{j}\right), \\
\delta^{k \times 4}(C \cdot \tilde{\eta}) & \equiv \prod_{a=1}^{k} \delta^{4}\left(\sum_{i=1}^{n+2} c_{a i} \tilde{\eta}_{i}\right),
\end{aligned}
$$

where the $C^{\perp}$ matrix is defined by identity $C \cdot\left(C^{\perp}\right)^{T}=0$ and it is assumed that all matrix manipulations are performed after $G L(k)$ gauge fixing. The delta functions above should be thought of as $\delta(x)=1 / x$, so that the corresponding contour integral computes the residue at $x=0$ [99]. Note that now arguments of delta functions are linear in integration variables $c_{a i}$. Also it is implemented that appropriate integration contour $\Gamma$ is chosen for $\mathrm{d}^{k \times(n+2)} C$ integration. We will label such a contour as $\Gamma_{k, n+2}^{\text {tree }}$. We will make some comments about explicit construction of $\Gamma_{k, n+2}^{\text {tree }}$ in the next section.

Next, by construction $F(C)$ contains $(k-2) \times(n-k)$ delta function factors forcing the integral over $C$ 's to have a Veronese form [26]. In general $F(C)$ is a rather complicated rational function of minors of the $C$ matrix; see the discussion in Sec. V. However, it could be shown that the choice of $F(C)$ in the form

$$
F(C)=\frac{1}{(1 \cdots k)(2 \cdots k+1) \cdots(n+2 \cdots k-1)}
$$

correctly reproduces the results of subsequent integration over Grassmannian ( $C$ matrixes). Here we use standard notations $\left(i_{1} \cdots i_{k}\right)$ to denote minors of the $C$ matrix constructed from columns of $C$ with numbers $i_{1}, \ldots, i_{k}{ }^{15}$ Because of this important step in our construction, we give

\footnotetext{
${ }^{15}$ We hope there will be no confusion with previous definition $(i j)=\sigma_{i \alpha} \sigma_{j}^{\alpha}$ used in $d^{2} \sigma_{a}$ integrals over homogeneous coordinates on Riemann sphere.
} 
a detailed discussion of this derivation, based on [99] in the next chapter.

Now we can perform a change of variables $c_{a i}$ that will simplify dependence on $\beta_{1}$ and $\beta_{2}$ in the integrand (linearize the dependence on $\beta_{1}$ and $\beta_{2}$ in the denominator), so that the integrals can be evaluated by residues. The computational details of this change of variables can be found in Appendix A.

To evaluate the residues we found it most convenient to use the notion of composite residue [22]. For that purpose let us define the set $S$ of points in $\mathbb{C}^{n}$, such that $S=\{z \mid z \in$ $\left.\mathbb{C}^{n}, s(z)=0\right\}$ and $s(z)$ is some holomorphic function (in our case polynomial). Next, consider $n$ form $\omega=h(z) /$ $s(z) d z$, where $d z=d z_{1} \wedge \cdots d z_{n}$ and $h(z)$ is some other holomorphic function (in our case it is some rational function), and define the $(n-1)$ form,

$$
\operatorname{res}_{j}[\omega]=\left.(-1)^{j-1}\left(\frac{h(z)}{\partial_{z_{j}} s(z)}\right)\right|_{S} d z_{[j]},
$$

with $\quad d z_{[j]}=d z_{1} \wedge \cdots \wedge d z_{j-1} \wedge d z_{j+1} \wedge \cdots \wedge d z_{n}$. Using this definition iteratively we may define $(n-m)$ forms as

$$
\operatorname{res}^{m}[\omega]=\operatorname{res}_{m} \circ \cdots \operatorname{ores}_{1}[\omega] \text {. }
$$

These forms are also known as composite residue forms. Considering our integral (4.16) as such a residue form

$$
\omega=\frac{\langle\xi p\rangle}{\kappa^{*}} \int \frac{\mathrm{d} \beta_{1} \wedge \beta_{2}}{\beta_{1} \beta_{2}} \int \frac{\mathrm{d}^{k \times(n+2)} C}{\operatorname{VolGL}(k)} \frac{\delta^{k \times 2}(C \cdot \underline{\underline{\lambda}}) \delta^{k \times 4}(C \cdot \underline{\underline{\tilde{\eta}}}) \delta^{(n+2-k) \times 2}\left(C^{\perp} \cdot \underline{\underline{\lambda}}\right)}{(1 \cdots k) \cdots(n-k+2 \cdots \mathbf{n}+\mathbf{1}) \cdots(\mathbf{n}+\mathbf{2} \cdots k-1)},
$$

where we used notations for minors of $C$ matrix,

$$
\begin{aligned}
& (\mathbf{n}+21 \cdots k-1)=\left(1+\beta_{1}\right)(n+11 \cdots k-1)+\beta_{1} \beta_{2}(n+21 \cdots k-1), \\
& (n-k+2 \cdots \mathbf{n}+\mathbf{1})=(n-k+2 \cdots n+1)+\beta_{2}(n-k+2 \cdots n n+2),
\end{aligned}
$$

and for kinematical variables

$$
\begin{array}{llll}
\underline{\underline{\lambda}}_{i}=\lambda_{i}, & i=1, \ldots n, & \underline{\underline{\lambda}}_{n+1}=\lambda_{p}, & \underline{\underline{\lambda}}_{n+2}=\xi, \\
\underline{\underline{\tilde{\lambda}}}_{i}=\tilde{\lambda}_{i}, & i=1, \ldots n, & \stackrel{\tilde{\tilde{\lambda}}}{=}_{n+1}=\frac{\langle\xi| k}{\langle\xi p\rangle}, & \underline{\tilde{\tilde{\lambda}}}_{n+2}=-\frac{\langle p| k}{\langle\xi p\rangle}, \\
\underline{\underline{\underline{\eta}}}_{i}=\tilde{\eta}_{i}, & i=1, \ldots n, & \stackrel{\tilde{\underline{\eta}}}{=}_{n+1}=\tilde{\eta}_{p}, & \underline{\underline{\tilde{\eta}}}_{n+2}=0,
\end{array}
$$

we can take residue as $\operatorname{res}_{\beta_{1}=-1} \circ \mathrm{res}_{\beta_{2}=0}$. This will give us the following result:

$$
\operatorname{res}_{\beta_{1}=-1} \operatorname{ores}_{\beta_{2}=0}[\omega]=A_{k, n+1}^{*},
$$

or more explicitly

$$
A_{k, n+1}^{*}=\int_{\Gamma_{k, n+2}^{\mathrm{ree}}} \frac{d^{k \times(n+2)} C}{\operatorname{Vol}[G L(k)]} \operatorname{Reg} \frac{\delta^{k \times 2}(C \cdot \underline{\tilde{\lambda}}) \delta^{k \times 4}(C \cdot \underline{\underline{\tilde{\eta}}}) \delta^{(n+2-k) \times 2}\left(C^{\perp} \cdot \underline{\underline{\lambda}}\right)}{(1 \cdots k) \cdots(n+1 \cdots k-2)(n+21 \cdots k-1)},
$$

where $^{16}$

$$
\operatorname{Reg}=\frac{\langle\xi p\rangle}{\kappa^{*}} \frac{(n+21 \cdots k-1)}{(n+11 \cdots k-1)} .
$$

This expression is in complete agreement with our previous derivation [63]. We will make some comments on the choice of integration contour $\Gamma_{k, n+2}^{\text {tree }}$ in Sec. V.

\footnotetext{
${ }^{16}$ Reg. notation for this combination of minors is chosen because such insertion regulates the behavior of $A_{k, n+1}^{*}$ with respect to the soft holomorphic limit in kinematical variables with labels $n+1$ or $n+2$ [63].
} 
Using (4.27) as the starting point we can perform the inverse operation-that is, taking partial integrations and reducing the integral in (5.8) to the integral over $\operatorname{Gr}(2, n+2)$ Grassmannian, ${ }^{17}$

$$
\begin{aligned}
A_{k, n+1}^{*}= & \int \prod_{a=1}^{n+2} \frac{\mathrm{d}^{2} \sigma_{a}}{(a a+1)} \frac{\operatorname{Reg} . V}{\operatorname{VolGL}(2, \mathbb{C})} \prod_{p=k+1}^{n+2} \bar{\delta}^{2}\left(\underline{\underline{\lambda}}_{p}-\underline{\underline{\lambda}}\left(\sigma_{p}\right)\right) \\
& \times \prod_{i=1}^{k} \bar{\delta}^{2 \mid 4}\left(\underline{\underline{\underline{\lambda}}} i-\underline{\underline{\underline{\lambda}}}\left(\sigma_{i}\right), \underline{\underline{\underline{\eta}}} i \underline{\underline{\tilde{\chi}}}\left(\sigma_{i}\right)\right)
\end{aligned}
$$

where $\operatorname{Reg}^{V}$ factor is given by

$$
\operatorname{Reg}^{V}=\frac{\langle\xi p\rangle}{\kappa^{*}} \frac{(k n+1)}{(k n+2)}
$$

and doubly underlined functions are defined as $(\underline{\underline{\lambda}}, \underline{\underline{\mu}}, \underline{\underline{\chi}})=\sum_{i=1}^{k} \frac{(\underline{\underline{\lambda}}, 0,0)}{\left(\sigma \sigma_{i}\right)}, \quad(\underline{\underline{\tilde{\mu}}}, \underline{\underline{\tilde{\lambda}}}, \underline{\underline{\tilde{\chi}}})=\sum_{p=k+1}^{n+2} \frac{(0, \underline{\underline{\tilde{\lambda}}}, \underline{\underline{\eta}} \underline{\underline{\eta}})}{\left(\sigma \sigma_{p}\right)}$

This should be equivalent to taking integrals over $\beta_{1}$ and $\beta_{2}$ directly in (4.11). Equation (4.29) can also be considered as RSV (scattering equation) representation for the Wilson line form factor. Scattering equations in this case are given by (3.24) where $\lambda(\sigma)$ 's and $\tilde{\lambda}(\sigma)$ 's are taken from (4.31).

The result for the case of amplitudes with multiple offshell legs $A_{k, m+n}^{*}$ could be obtained along the same lines as previous discussions. In the case with first $m$ particles onshell and last $n$ being off-shell, making identical assumptions as in the $n=1$ case about the form of the $F(C)$ function, we would get for $A_{k, m+n}^{*}$,

$$
\begin{aligned}
A_{k, m+n}^{*}= & \int_{\Gamma_{k, m+2 n}^{\mathrm{ree}}} \frac{d^{k \times(m+2 n)} C}{\operatorname{Vol}[G L(k)]} \operatorname{Reg}(m+1, \ldots, m+n) \\
& \times \frac{\delta^{k \times 2}(C \cdot \underline{\underline{\lambda}}) \delta^{k \times 4}(C \cdot \underline{\underline{\tilde{\eta}}}) \delta^{(m+2 n-k) \times 2}\left(C^{\perp} \cdot \underline{\underline{\lambda}}\right)}{(1 \cdots k) \cdots(m \cdots m+k-1)(m+1 \cdots m+k) \cdots(m+2 n \cdots k-1)},
\end{aligned}
$$

where the external kinematical variables are chosen as

$$
\begin{aligned}
& \underline{\lambda}_{i}=\lambda_{i}, \quad i=1, \ldots, m, \quad \underline{\lambda}_{m+2 j-1}=\lambda_{p_{j}}, \quad \underline{\lambda}_{m+2 j}=\xi_{j}, \quad j=1, \ldots, n, \\
& \underline{\tilde{\lambda}}_{i}=\tilde{\lambda}_{i}, \quad i=1, \ldots, m, \quad \underline{\tilde{\lambda}}_{m+2 j-1}=\frac{\left\langle\xi_{j}\right| k_{m+j}}{\left\langle\xi_{j} p_{j}\right\rangle}, \quad \underline{\tilde{\lambda}}_{m+2 j}=-\frac{\left\langle p_{j}\right| k_{m+j}}{\left\langle\xi_{j} p_{j}\right\rangle}, \quad j=1, \ldots, n, \\
& \underline{\underline{\tilde{\eta}}}_{i}=\tilde{\eta}_{i}, \quad i=1, \ldots, m, \quad \underline{\underline{\eta}}_{m+2 j-1}=\tilde{\eta}_{p_{j}}, \quad \underline{\tilde{\eta}}_{m+2 j}=0, \quad j=1, \ldots, n,
\end{aligned}
$$

and the $\operatorname{Reg}(m+1, \ldots, m+n)$ function is given by the products of ratios of minors of the $C$ matrix:

$$
\begin{aligned}
\operatorname{Reg}(m+1, \ldots, m+n) & =\prod_{j=1}^{n} \operatorname{Reg}(j+m), \\
\operatorname{Reg}(j+m) & =\frac{\left\langle\xi_{j} p_{j}\right\rangle}{\kappa_{j}^{*}} \frac{(2 j+m 2 j+1+m \cdots 2 j+k-1+m)}{(2 j-1+m 2 j+1+m \cdots 2 j+k-1+m)} .
\end{aligned}
$$

This result also coincides with the Grassmannian integral representation for $A_{k, m+n}^{*}$ first conjectured in [64]. Also note that if the number of $\mathcal{V}_{i, i+1}^{\mathrm{WL}}$ operators is greater than $\tilde{\mathcal{V}}_{i}$, which is given by $k$, then the correlation function is equal to 0 . $\tilde{\mathcal{V}}_{i}$ from which $\mathcal{V}_{i, i+1}^{\mathrm{WL}}$ may be constructed also are taken into account. It is also possible to rewrite this result in RSV (scattering equation) form:

$$
\begin{aligned}
A_{k, m+n}^{*}= & \int \prod_{a=1}^{n+2} \frac{\mathrm{d}^{2} \sigma_{a}}{(a a+1)} \frac{\operatorname{Reg}^{V}(m+1, \ldots, m+n)}{\operatorname{VolGL}(2, \mathbb{C})} \prod_{p=k+1}^{m+2 n} \bar{\delta}^{2}\left(\underline{\underline{\lambda}}_{p}-\underline{\underline{\lambda}}\left(\sigma_{p}\right)\right) \\
& \times \prod_{i=1}^{k} \bar{\delta}^{2 \mid 4}\left(\underline{\underline{\tilde{\lambda}}}_{i}-\underline{\tilde{\lambda}}\left(\sigma_{i}\right), \underline{\underline{\eta}} i-\underline{\underline{\tilde{\chi}}}=\right.
\end{aligned}
$$

\footnotetext{
${ }^{17} \operatorname{The} \operatorname{Gr}(2, n+2)$ Grassmannian is embedded into the $\operatorname{Gr}(k, n+2)$ Grassmannian again with the help of the Veronese map; see, for example, [70].
} 
where

$$
\operatorname{Reg}^{V}(m+1, \ldots, m+n)=\prod_{j=1}^{n} \operatorname{Reg}^{V}(j+m), \quad \operatorname{Reg}^{V}(j+m)=\frac{\left\langle\xi_{j} p_{j}\right\rangle}{\kappa_{j}^{*}} \frac{(k 2 j-1+m)}{(k 2 j+m)}
$$

and doubly underlined functions of $\sigma$ as before are defined in (4.31). Note that now doubly underlined kinematical variables in (4.31) should be taken from (4.33). At the end, we want to stress that the explicit form of (4.30) and (4.36) is not unique and is in fact related to the $G L(k)$ gauge choice in (4.27) and (4.32).

\section{B. Generalized world-sheet vertex operators for $\mathcal{N}=4$ SYM field theory stress-tensor supermultiplet operators insertion}

Let us now consider different choice of target space wave functions $\psi$. Namely, let us choose $\psi$ as

$$
\psi\left(\left\{\lambda_{j}, \tilde{\lambda}_{j}, \eta_{j}\right\}_{j=i}^{i+1},\left\{q, \gamma^{-}\right\}\right)=F_{2,2}\left(\Omega_{n}, \Omega_{n+1} ; \mathcal{T}\right) \times \text { color projectors }
$$

Here $F_{2,2}$ is the minimal form factor of operators from the $\mathcal{N}=4 \mathrm{SYM}$ stress tensor supermultiplet. ${ }^{18}$ This choice of target space wave function should correspond to the world-sheet generalized vertex operator $\mathcal{V}^{\mathrm{ST}}$ which should describe the insertions of operators from the $\mathcal{N}=4 \mathrm{SYM}$ stress-tensor supermultiplet in the on-shell amplitude, i.e., the corresponding form factor

$$
\mathcal{V}_{i, i+1}^{\mathrm{ST}}=\left.\left.\int \prod_{j=i}^{i+1} \frac{\mathrm{d}^{2} \lambda_{j} \mathrm{~d}^{2} \tilde{\lambda}_{j}}{\operatorname{Vol}[\mathrm{GL}(1)]} \mathrm{d}^{4} \tilde{\eta}_{j} F_{2,2}\left(\Omega_{i}, \Omega_{i+1} ; \mathcal{T}\right)\right|_{\lambda \rightarrow-\lambda} \mathcal{V}_{i} \mathcal{V}_{i+1}\right|_{T^{a} T^{b} \rightarrow \delta^{a b} \rightarrow 1}
$$

where, as in the previous case, the vertex is supposed to be inserted at points $\sigma_{i}, \sigma_{i+1}$ and we have used projection of the tensor product of two adjoint on-shell state color representations onto singlet color representation. Note that the initial correlation function of vertex operators (3.16) is a colored object. The singlet projection considered here will effectively lead to the situation when on the level of color ordered objects we will have to consider all possible positions ${ }^{19}$ of $\mathcal{V}_{i, i+1}^{\mathrm{sT}}$ ("gluing positions") starting from $i=1$ up to $i=n+1$. The minimal form factor $F_{2,2}\left(\Omega_{i}, \Omega_{i+1} ; \mathcal{T}\right)$ itself is given by [74]

$$
F_{2,2}\left(\Omega_{i}, \Omega_{i+1} ; \mathcal{T}\right)=\delta^{2}\left(\underline{\tilde{\lambda}}_{i}\right) \delta^{4}\left(\underline{\tilde{\eta}}_{i}\right) \delta^{2}\left(\underline{\tilde{\lambda}}_{i+1}\right) \delta^{4}\left(\underline{\tilde{\eta}}_{i+1}\right)
$$

with ( $q$ and $\gamma^{-}$are the operator's momentum and supermomentum correspondingly)

$$
\begin{gathered}
\tilde{\tilde{\lambda}}_{i}=\tilde{\lambda}_{i}-\frac{\langle i+1| q}{\langle i+1 i\rangle}, \quad \underline{\tilde{\eta}}_{i}^{-}=\tilde{\eta}_{i}^{-}-\frac{\langle i+1| \gamma^{-}}{\langle i+1 i\rangle}, \quad \underline{\tilde{\eta}}_{i}^{+}=\tilde{\eta}_{i}^{+}, \\
\underline{\tilde{\lambda}}_{i+1}=\tilde{\lambda}_{i+1}-\frac{\langle i| q}{\langle i i+1\rangle}, \quad \underline{\tilde{\eta}}_{i+1}^{-}=\tilde{\eta}_{i+1}^{-}-\frac{\langle i| \gamma^{-}}{\langle i i+1\rangle}, \quad \underline{\tilde{\eta}}_{i+1}^{+}=\tilde{\eta}_{i+1}^{+} .
\end{gathered}
$$

Integrating over helicity spinors $\lambda_{i}, \tilde{\lambda}_{i}$ we get

$$
\mathcal{V}_{i, i+1}^{\mathrm{ST}}=-\left.\left\langle\xi_{A} \xi_{B}\right\rangle^{2} \int \mathrm{d} \beta_{1} \int \mathrm{d} \beta_{2} \mathcal{V}_{i} \mathcal{V}_{i+1}\right|_{T^{a} T^{b} \rightarrow \delta^{a b} \rightarrow 1},
$$

where

$$
\lambda_{i}=\xi_{A}-\beta_{1} \xi_{B}, \quad \tilde{\lambda}_{i}=\frac{1}{\beta_{1} \beta_{2}-1} \frac{\left\langle\xi_{B}\right| q}{\left\langle\xi_{B} \xi_{A}\right\rangle}+\frac{\beta_{2}}{\beta_{1} \beta_{2}-1} \frac{\left\langle\xi_{A}\right| q}{\left\langle\xi_{A} \xi_{B}\right\rangle}
$$

\footnotetext{
${ }^{18}$ More accurately it is chiral truncation [91].

${ }^{19}$ Let us remind the reader that we consider combinations $\mathcal{V}_{i} \mathcal{V}_{i+1}, \mathcal{V}_{i} \tilde{\mathcal{V}}_{i+1}$, and $\tilde{\mathcal{V}}_{i} \tilde{\mathcal{V}}_{i+1}$ in the definition of $\mathcal{V}_{i, i+1}^{\mathrm{ST}}$ on equal footing.
} 


$$
\lambda_{i+1}=\xi_{B}-\beta_{2} \xi_{A}, \quad \tilde{\lambda}_{i+1}=\frac{1}{\beta_{1} \beta_{2}-1} \frac{\left\langle\xi_{A}\right| q}{\left\langle\xi_{A} \xi_{B}\right\rangle}+\frac{\beta_{1}}{\beta_{1} \beta_{2}-1} \frac{\left\langle\xi_{B}\right| q}{\left\langle\xi_{B} \xi_{A}\right\rangle}
$$

and

$$
\begin{array}{cc}
\tilde{\eta}_{i}^{-}=\frac{1}{\beta_{1} \beta_{2}-1} \frac{\left\langle\xi_{B}\right| \gamma^{-}}{\left\langle\xi_{B} \xi_{A}\right\rangle}+\frac{\beta_{2}}{\beta_{1} \beta_{2}-1} \frac{\left\langle\xi_{A}\right| \gamma^{-}}{\left\langle\xi_{A} \xi_{B}\right\rangle}, \quad \tilde{\eta}_{i}^{+}=0, \\
\tilde{\eta}_{i+1}^{-}=\frac{1}{\beta_{1} \beta_{2}-1} \frac{\left\langle\xi_{A}\right| \gamma^{-}}{\left\langle\xi_{A} \xi_{B}\right\rangle}+\frac{\beta_{1}}{\beta_{1} \beta_{2}-1} \frac{\left\langle\xi_{B}\right| \gamma^{-}}{\left\langle\xi_{B} \xi_{A}\right\rangle}, \quad \tilde{\eta}_{i+1}^{+}=0 .
\end{array}
$$

The evaluation of the string correlation function with stress-tensor vertex operator insertion (here we are a considering color ordered object)

$$
F_{k, n}=\left\langle\tilde{\mathcal{V}}_{1} \cdots \tilde{\mathcal{V}}_{k} \mathcal{V}_{k+1} \cdots \mathcal{V}_{n} \mathcal{V}_{n+1, n+2}^{\mathrm{ST}}\right\rangle+\text { other gluing positions }
$$

closely follows the corresponding calculation for the case of the Wilson line vertex operator insertion presented above. We also want to simplify arguments of delta functions. For that purpose we introduce unity decomposition [26] in the form (4.12) and use conjecture that $F(C)$ can be chosen as (4.19). This give us the following expression:

$$
\begin{aligned}
F_{k, n}= & -\left\langle\xi_{A} \xi_{B}\right\rangle^{2} \int \mathrm{d} \beta_{2} \mathrm{~d} \beta_{1} \int \frac{\mathrm{d}^{k \times(n+2)} C}{\operatorname{Vol}[G L(k)]} F(C) \delta^{k \times 2}(C \cdot \tilde{\lambda}) \delta^{k \times 4}(C \cdot \tilde{\eta}) \delta^{(n+2-k) \times 2}\left(C^{\perp} \cdot \lambda\right) \\
& + \text { other gluing positions. }
\end{aligned}
$$

Note once more that here in the expression above in all terms $\lambda_{i}, \tilde{\lambda}_{i}, \tilde{\eta}_{i}$ are functions of $\beta_{1}$ and $\beta_{2}$ according to (4.43)-(4.46). In the first term explicitly written here $i=n+1$, in the second $i=n$, etc. After an appropriate change of variables, which is given in Appendix A, we can rewrite (4.48) as (similar to the previous case we understand integration over $\beta_{1}, \beta_{2}$ as residue form $\omega$, though in this case it does not bring any simplifications)

$$
\begin{aligned}
\omega= & -\left\langle\xi_{A} \xi_{B}\right\rangle^{2} \int \frac{d \beta_{1} \wedge d \beta_{2}}{\left(1-\beta_{1} \beta_{2}\right)} \int \frac{d^{k \times(n+2)} C}{\operatorname{Vol}[G L(k)]} \delta^{k \times 2}\left(C^{\prime} \cdot \underline{\underline{\lambda}}\right) \delta^{k \times 4}\left(C^{\prime} \cdot \underline{\underline{\tilde{\eta}}}\right) \delta^{(n+2-k) \times 2}\left(C^{\perp \perp} \cdot \underline{\underline{\lambda}}\right) \\
& \times \frac{1}{(1 \cdots k) \cdots(n-k+2 \cdots n \mathbf{n}+\mathbf{1}) \cdots(\mathbf{n}+\mathbf{2} 1 \cdots k-1)}+\text { other gluing positions. }
\end{aligned}
$$

Here the following notations were used for minors:

$$
\begin{aligned}
(n-k+2 \cdots n \mathbf{n}+\mathbf{1}) & =(n-k+2 \cdots n \mathbf{n}+\mathbf{1})-\beta_{1}(n-k+2 \cdots n n+2), \\
(\mathbf{n}+\mathbf{2} 1 \cdots k-1) & =(n+21 \cdots k-1)-\beta_{2}(n+11 \cdots k-1),
\end{aligned}
$$

and for kinematical variables,

$$
\begin{array}{lll}
\underline{\lambda}_{i}=\lambda_{i}, & i=1, \ldots, n, & \underline{\lambda}_{n+1}=\xi_{A}, \quad \underline{\underline{\lambda}}_{n+2}=\xi_{B}, \\
\underline{\tilde{\lambda}}_{i}=\tilde{\lambda}_{i}, & i=1, \ldots, n, \quad & \underline{\tilde{\lambda}}_{n+1}=-\frac{\left\langle\xi_{B}\right| q}{\left\langle\xi_{B} \xi_{A}\right\rangle}, \quad \underline{\tilde{\lambda}}_{n+2}=-\frac{\left\langle\xi_{A}\right| q}{\left\langle\xi_{A} \xi_{B}\right\rangle}, \\
\underline{\tilde{\eta}}_{i}^{+}=\tilde{\eta}_{i}^{+}, & i=1, \ldots, n, & \underline{\tilde{\eta}}_{n+1}^{+}=0, \quad \underline{\underline{\eta}}_{n+2}^{+}=0, \\
\underline{\tilde{\eta}}_{i}^{-}=\tilde{\eta}_{i}^{-}, & i=1, \ldots, n, & \quad \underline{\tilde{\eta}}_{n+1}^{-}=-\frac{\left\langle\xi_{B}\right| \gamma^{-}}{\left\langle\xi_{B} \xi_{A}\right\rangle}, \quad \underline{\tilde{\eta}}_{n+2}^{-}=-\frac{\left\langle\xi_{A}\right| \gamma^{-}}{\left\langle\xi_{A} \xi_{B}\right\rangle} .
\end{array}
$$

Taking residues at $\beta_{1}^{*}=\frac{(n-k+2 \cdots n n+1)}{(n-k+2 \cdots n n+2)}$ and $\beta_{2}^{*}=\frac{(n+21 \cdots k-1)}{(n+11 \cdots k-1)}$ we reproduce the result of [74] (computational details can also be found in Appendix A) 


$$
F_{k, n}=\operatorname{res}_{\beta_{1}=\beta_{1}^{*}} \operatorname{rres}_{\beta_{2}=\beta_{1}^{*}}[\omega]
$$

where (here we write explicitly only the first term corresponding to $\mathcal{V}_{i, i+1}^{\mathrm{ST}}$ positioned in $i=n+1$ )

$$
\begin{aligned}
F_{k, n}= & \int_{\Gamma_{k, n+2}^{\text {treen+1 }}} \frac{d^{k \times(n+2)} C}{\operatorname{Vol}[G L(k)]} \operatorname{Reg} \frac{\delta^{k \times 2}(C \cdot \underline{\underline{\tilde{\lambda}}}) \delta^{k \times 4}(C \cdot \underline{\underline{\tilde{\eta}}}) \delta^{(n+2-k) \times 2}\left(C^{\perp} \cdot \underline{\underline{\lambda}}\right)}{(1 \cdots k)(2 \cdots k+1) \cdots(n+2 \cdots k-1)} \\
& + \text { other gluing positions, }
\end{aligned}
$$

with $^{20}$

$$
\operatorname{Reg}=\left\langle\xi_{A} \xi_{B}\right\rangle^{2} \frac{Y}{1-Y}, \quad Y=\frac{(n-k+2 \cdots n n+1)(n+21 \cdots k-1)}{(n-k+2 \cdots n n+2)(n+11 \cdots k-1)}
$$

The additional label in $\Gamma_{k, n+2}^{\mathrm{tree}, n+1}$ corresponds to the fact that for each term corresponding to different $\mathcal{V}_{i, i+1}^{\mathrm{ST}}$ positions integration contours should be, in general, chosen separately [74].

Using expression (4.53) obtained above as in the previous discussion, we can perform the inverse operation-that is, take partial integrations and reduce the integral in (5.8) to the integral over $\operatorname{Gr}(2, n+2)$ Grassmannian

$$
\begin{aligned}
F_{k, n}= & \int \prod_{a=1}^{n+2} \frac{\mathrm{d}^{2} \sigma_{a}}{(a a+1)} \frac{\operatorname{Reg}}{\operatorname{VolGL}(2, \mathbb{C})} \prod_{p=k+1}^{n+2} \bar{\delta}^{2}\left(\underline{\underline{\lambda}} p-\underline{\underline{\lambda}}\left(\sigma_{p}\right)\right) \prod_{i=1}^{k} \bar{\delta}^{2 \mid 4}\left(\underline{\underline{\lambda}} i-\underline{\underline{\lambda}}\left(\sigma_{i}\right), \underline{\underline{\eta}} i-\underline{\underline{\tilde{\chi}}}\left(\sigma_{i}\right)\right) \\
& + \text { other gluing positions, }
\end{aligned}
$$

where the Reg factor is now given by [70]

$$
\operatorname{Reg}=\left\langle\xi_{A} \xi_{B}\right\rangle^{2} \frac{Y}{1-Y}, \quad Y=\prod_{j=n+2-k}^{n} \frac{(j n+1)}{(j n+2)} \prod_{i=1}^{k-1} \frac{(n+2 i)}{(n+1 i)}
$$

and doubly underlined functions are defined as in the case of Wilson line insertion,

$$
(\underline{\underline{\lambda}}, \underline{\underline{\mu}}, \underline{\underline{\chi}})=\sum_{i=1}^{k} \frac{(\underline{\underline{\lambda}}, 0,0)}{\left(\sigma \sigma_{i}\right)}, \quad(\underline{\underline{\tilde{\mu}}}, \underline{\underline{\lambda}}, \underline{\underline{\tilde{\chi}}})=\sum_{p=k+1}^{n+2} \frac{(0, \underline{\underline{\underline{\lambda}}} p, \underline{\underline{\underline{\eta}}} p)}{\left(\sigma \sigma_{p}\right)}
$$

This should be equivalent to direct calculation of $\beta_{1,2}$ integrals in (4.10). This also can be considered as an analog of RSV (scattering equation) representation of form factors of the stress tensor supermultiplet operator.

Let us remind the reader once more that in the formula above the term + other gluing positions denotes all other insertion positions of the minimal form factor in the color ordered on-shell amplitude. Note that the original string correlation function contains all these terms corresponding to different gluing positions from the very beginning.

At the and of this section let us make the following comment regarding results presented in the literature

\footnotetext{
${ }^{20}$ Similar to the previous case Reg insertion regulates the soft holomorphic limit with respect to kinematical variables with labels $n+1$ and $n+2$ [73].
}

$[70,100]$. Scattering equation representation obtained here is different from the main result of [70] (see 2.11 there). We want to stress that we reproduce results of [74] starting from the vertex operator correlation function and our definition of generalized vertex operator (4.37), at least if we fix "appropriate" integration order and will take integrals with respect to $\beta_{1,2}$ in (4.48) as the last one. Scattering equation representation (4.55) also obviously coincides with the results of the application of the Veronese map to individual terms of Grassmannian representation of [74]. We also have checked that we reproduce answers for next to maximally helicity violating (NMHV) $n=3,4,5$ point and next to next to maximally helicity violating (NNMHV) $n=4$ point form factors, similar to [74] if we use the integration ordering described above. We think that both scattering equation representations, i.e., [70] and (4.55), give in the end (after integration) identical results, and the explanation to this is that the different functions can have a coinciding subset of residues.

\section{Symmetry properties of form factors and correlation functions in $\mathcal{N}=4 \mathrm{SYM}$}

Here we want to share some observations regarding the properties of the results obtained for the form factors and correlation functions with respect to dual conformal 
symmetry. It is known [31] that the tree level amplitudes in $\mathcal{N}=4$ SYM are invariant with respect to dual conformal transformations, which can be considered as the consequence of integrability of $\mathcal{N}=4$ SYM [32,34]. At first glance, the results obtained in $[63,64]$ and reproduced here in (4.27) and (4.32) are in general not dual conformal invariant. The dual conformal invariance will be restored only in the soft holomorphic limit $[53,63]$ with respect to auxiliary on-shell momenta $p_{\xi}=\xi \tilde{\xi}$. To illustrate this statement let us transform (4.27) into momentum twistor representation [63]. The result will be given by

$$
\begin{aligned}
\frac{A_{k, n+1}^{*}}{A_{2, n+1}^{*}} & =\omega_{n+2}^{k}\left[\Gamma_{k, n+2}\right], \\
\omega_{n+2}^{k}[\Gamma] & =\int_{\Gamma} \frac{d^{(k-2) \times(n+2)} D}{\operatorname{Vol}[G L(k-2)]} \frac{1}{1+\frac{\langle p \xi\rangle}{\langle p 1\rangle}\left(\frac{(n+22 \cdots k-2)}{(1 \cdots k-2)}\right.} \frac{\delta^{4(k-2) \mid 4(k-2)}(D \cdot \mathcal{Z})}{(1 \cdots k-2) \cdots(n+2 \cdots k-3)},
\end{aligned}
$$

with

$$
\delta^{4(k-2) \mid 4(k-2)}(D \cdot \mathcal{Z})=\prod_{a=1}^{k-2} \delta^{4 \mid 4}\left(\sum_{i=1}^{n+2} D_{a i} \mathcal{Z}_{i}\right),
$$

where momentum (super)twistors $\mathcal{Z}_{1}, \ldots, \mathcal{Z}_{n}$ describe the on-shell momenta of particles and $\mathcal{Z}_{n+1}, \mathcal{Z}_{n+2}$ describe the off-shell momenta carried by the Wilson line operator insertion [63,74]. Their bosonic components are given by $\left(\lambda_{p}, x_{n+1} \lambda_{p}\right)$ and $\left(\xi, x_{n+2} \xi\right)$ correspondingly. Factor $\langle p \xi\rangle /\langle p 1\rangle$ can be written as a product of the bosonic components of $\mathcal{Z}_{1}$ and $\mathcal{Z}_{n+1}, \mathcal{Z}_{n+2}$ with infinity twistor $I_{A B}$ [35], which, at first glance, is explicit indication of the breakdown of dual conformal invariance. A similar situation also occurs for the stress tensor supermultiplet form factors [74].

Note, however, that the Wilson line form factors and correlation functions should be independent of the particular choice $|\xi\rangle[53,63]$. This allows one to choose $|\xi\rangle=|1\rangle$ and cancel out the $\langle p \xi\rangle /\langle p 1\rangle$ factor. As an explicit example let us consider the ratio $A_{3,4+1}^{*} / A_{2,4+1}^{*}$ of the form factors written in momentum twistor representation (see [63] for details):

$$
\frac{A_{3,4+1}^{*}}{A_{2,4+1}^{*}}\left(\Omega_{1}, \ldots, \Omega_{4}, g_{5}^{*}\right)=\frac{1}{1+\frac{\langle p \xi\rangle}{\langle p 1\rangle} \frac{\langle 1345\rangle}{\langle 3456\rangle}}[13456]+\frac{1}{1+\frac{\langle p \xi\rangle}{\langle p 1\rangle} \frac{\langle 1235\rangle}{\langle 2356\rangle}}[12356]+[12345] .
$$

After substitution $|\xi\rangle=|1\rangle$ we obtain

$$
\frac{A_{3,4+1}^{*}}{A_{2,4+1}^{*}}\left(\Omega_{1}, \ldots, \Omega_{4}, g_{5}^{*}\right)=\frac{1}{1+\frac{\langle 1345\rangle}{\langle 3456\rangle}}[13456]+\frac{1}{1+\frac{\langle 1235\rangle}{\langle 2356\rangle}}[12356]+[12345],
$$

which should be dual conformal invariant. Note that in the expression above $Z_{6}=\left(\lambda_{1}, x_{6} \lambda_{1}\right)$, due to the choice of $|\xi\rangle$, so $Z_{6}$ scales identical to $Z_{1}$ and the ratios $\langle 1345\rangle /\langle 6345\rangle$, $\langle 1235\rangle /\langle 6235\rangle$ should be dual conformal invariant.

Such a choice of $|\xi\rangle$, however, may be singular in a sense that in the intermediate expression when one transforms (4.27) into (4.58) one can encounter $0 / 0$ uncertainties. Note also that the same considerations as above are also true for the stress tensor supermultiplet form factors [74].

In the light of recent results $[101,102]$ these observations still give us hope that the dual conformal invariance can be observed, in some form, in all correlation functions and form factors of the gauge invariant operators including the cases considered here.

\section{GRASSMANNIANS, SCATTERING EQUATIONS, AND LINK REPRESENTATIONS}

In derivation of Grassmannian representations (4.27) and (4.53) from ambitwistor string world-sheet correlation functions (4.10) and (4.47) it was crucial that we can choose the $F(C)$ function in the form of (4.19). So for selfconsistency here we want to present a detailed discussion and give arguments that such a choice is indeed possible. Our discussion will be based mostly on [26], so if the reader is familiar with the content of [26] he/she can skip reading to the end of this section where explicit example is considered for the Wilson line field theory operator form factor with $n+2=6$ and $k=3$. 
Let us look at the case of the Wilson line operator form factors and consider (4.16). For stress tensor supermultiplet operator form factors we will have a similar expression but with different $\beta_{1,2}$ dependence. For fixed values of $\beta_{1,2}$ parameters the integrand of (4.16) looks like

$$
\begin{aligned}
\operatorname{Int}= & \int \frac{\mathrm{d}^{k \times(n+2)} C}{\operatorname{VolGL}(k)} F(C) \delta^{k \times 2}(C \cdot \tilde{\lambda}) \delta^{k \times 4}(C \cdot \tilde{\eta}) \\
& \times \delta^{(n+2-k) \times 2}\left(C^{\perp} \cdot \lambda\right) .
\end{aligned}
$$

All dependence on $\beta_{1,2}$ is accumulated in this expression in $\left\{\lambda_{i}, \tilde{\lambda}_{i}, \eta_{i}\right\}_{i=n+1}^{n+2}$ and is given by (4.7). Here

$$
\begin{aligned}
F(C)= & \int \frac{1}{\operatorname{VolGL}(2, \mathbb{C})} \prod_{b=1}^{n} \frac{\mathrm{d} s_{b} \mathrm{~d} \sigma_{b}}{s_{a}\left(\sigma_{b}-\sigma_{b+1}\right)} \\
& \times \prod_{\substack{a \in f \\
i \in g}} \delta\left(c_{a i}-\frac{s_{a} s_{i}}{\sigma_{a}-\sigma_{i}}\right)
\end{aligned}
$$

with $f, g$ denoting index sets $f=1, \ldots, k$ and $g=k+1$, $\ldots, n+2^{21}$ Note that $F(C)$ is completely kinematically independent and will have the same form also for the case of stress tensor supermultiplet form factors. In fact Int is identical to the RSV representation of the $n+2$ point $\mathrm{N}^{k-2} \mathrm{MHV}$ amplitude: $\mathrm{Int}=A_{k, n+2}$ (it is implemented that appropriate integration contour $\Gamma$ is chosen) with appropriately chosen kinematics. So let us forget for now about $\beta_{1,2}$ integrals completely and concentrate on the RSV representation of $A_{k, n+2}$. Let us transform the expression for (5.1) into a form more suitable for our purpose. For that it is convenient to rearrange delta functions of kinematical constraints in the form [22]

$$
\begin{aligned}
\delta^{k \times 2}(C \cdot \tilde{\lambda}) \delta^{(n+2-k) \times 2}\left(C^{\perp} \cdot \lambda\right) \\
=\delta^{4}\left(\sum_{j=1}^{n+2} \lambda_{j} \tilde{\lambda}_{j}\right) J(\lambda, \tilde{\lambda}) \\
\quad \times \int d^{(k-2)(n-k)} \tau_{A} \prod_{\substack{a \in f \\
i \in g}} \delta\left(c_{a i}-c_{a i}(\tau \mid \operatorname{kin})\right),
\end{aligned}
$$

where $J(\lambda, \tilde{\lambda})$ is the Jacobian of transformation and $c_{a i}(\tau \mid$ kin $)$ is a general solution of the underdetermined system of linear equations [22,103]

\footnotetext{
${ }^{21}$ This particular form of $f$ and $g$ is related to the $G L(k)$ gauge choice. Namely, $f$ contains the numbers of columns constituting the unity matrix. The different choices of $f$ and $g$ sets with given total numbers of elements $\# f=k, \# g=n-k+2$ in each set correspond to different gauge choices and also to different rearrangements of $\mathcal{V}_{a}$ and $\tilde{\mathcal{V}}_{a}$ vertex operators among themselves in the correlation function. All gauges should lead to the same result.
}

$$
\begin{gathered}
c_{a i} \lambda_{a}=-\lambda_{i}, \\
c_{a i} \tilde{\lambda}_{i}=-\tilde{\lambda}_{a},
\end{gathered}
$$

with $a \in f, i \in g$. The solution depends on external kinematical data $\lambda_{i}, \tilde{\lambda}_{i}$ as well as on the arbitrary $(k-2)(n-k)$ parameters $\tau_{A}$. The explicit form of $c_{a i}(\tau \mid$ kin $)$ for general $n$ and $k$ can be found in [103]. For example [22], for $n+2=6, k=3, f \in(1,3,5)$, and $g \in(2,4,6)$, we have $c_{a i}(\tau \mid \operatorname{kin})=c_{a i}^{*}+\epsilon_{a a_{1} a_{2}} \epsilon_{i i_{1} i_{2}}\left\langle a_{1} a_{2}\right\rangle\left[i_{1} i_{2}\right] \tau$, where $c_{a i}^{*}$ is some particular solution of (5.4). Using the representation (5.3) we can remove integration over $d^{k \times(n+2)} C / \operatorname{Vol}[G L(k)]$. Next, let us for simplicity fix helicities of external particles in such a way that Grassmann delta functions $\delta^{k \times 4}$ go to $1 .^{22}$ All these manipulations reduce our initial expression (5.1) to

$$
\begin{aligned}
A_{k, n+2}= & \delta^{4}\left(\sum_{j=1}^{n+2} \lambda_{j} \tilde{\lambda}_{j}\right) J(\lambda, \tilde{\lambda}) \\
& \times\left.\int_{\Gamma} d^{(k-2)(n-k)} \tau_{A} F(C)\right|_{c_{a i} \mapsto c_{a i}(\tau \mid \mathrm{kin})},
\end{aligned}
$$

with the appropriate choice of integration contour $\Gamma$.

Now one can try to evaluate the function $F(C)$ for general values of $n$ and $k$ in terms of matrix elements $c_{a i}$ [103]. It is a rather complicated expression. The most studied case is $k=3[4,26,104]$, and it is believed that for $k>3$ the behavior will be essentially the same as in the $k=3$ case [26]. Then let us also concentrate on the $k=3$ case as representative, yet a simple enough example. In this case we can rewrite the $F(C)$ function in terms of minors of the $C$ matrix and get $[26,104]$

$$
\begin{aligned}
F^{k=3}(C) & =H(C) \frac{1}{S_{6} \cdots S_{n+2}}, \\
H(C) & =\frac{\prod_{j=6}^{n+1}(12 j)(23 j-1) \prod_{i=5}^{n+1}(13 i)}{(n+1 n+21)(123)(234)},
\end{aligned}
$$

and $(j=6, \ldots, n+2)$

$$
\begin{aligned}
S_{j}= & (j-2 j-1 j)(j 12)(23 j-2)(j-113) \\
& -(j-1 j 1)(123)(3 j-2 j-1)(j 2 j-2) .
\end{aligned}
$$

Note that it is different from our choice (4.19).

Let us summarize what we have learned so far. We have explicitly evaluated integral (5.2) for $k=3$ and found that the result of evaluation is naively different from what we have conjectured. Presumably $k>3$ will be no better.

\footnotetext{
${ }^{22}$ This is always possible for appropriate $G L(k)$ gauge and external state choices. For example, for $n+2=6, k=3$, and $f=2,4,6, g=1,3,5$ the appropriate choice of the external particle helicities will be $(+-+-+-)$ [22].
} 
To see how this contradiction resolves, let us consider representation of the amplitude $A_{k, n+2}$ in terms of the integral over Grassmannian $\operatorname{Gr}(k, n+2)$

$$
\begin{aligned}
A_{k, n+2}= & \int_{\Gamma_{k, n+2}^{\mathrm{tree}}} \frac{d^{k \times(n+2)} C}{\operatorname{Vol}[G L(k)]} \\
& \times \frac{\delta^{k \times 2}(C \cdot \underline{\underline{\tilde{\lambda}}}) \delta^{k \times 4}(C \cdot \underline{\underline{\tilde{\eta}}}) \delta^{(n+2-k) \times 2}\left(C^{\perp} \cdot \underline{\underline{\lambda}}\right)}{(1 \cdots k) \cdots(n+1 \cdots k-2)(n+21 \cdots k-1)} .
\end{aligned}
$$

Using the same manipulations as before [namely, (5.3) and (5.4)] we will arrive at a similar expression (5.5) [26], but with different forms of the $F(C)$ function, which we will denote now as $F_{G r}(C)$,

$$
\begin{aligned}
A_{n+2, k}= & \delta^{4}\left(\sum_{j=1}^{n+2} \lambda_{j} \tilde{\lambda}_{j}\right) J(\lambda, \tilde{\lambda}) \\
& \times\left.\int_{\Gamma_{k, n+2}^{\mathrm{ree}}} d^{(k-2)(n-k)} \tau_{A} F_{G r}(C)\right|_{c_{a i} \mapsto c_{a i}(\tau \mid \mathrm{kin})},
\end{aligned}
$$

where for $k=3$

$$
\begin{aligned}
F_{\mathrm{Gr}}^{k=3}(C) & =\tilde{H}(C) \frac{1}{\tilde{S}_{6} \cdots \tilde{S}_{n+2}}, \\
\tilde{H}(C) & =\frac{\prod_{j=6}^{n+1}(12 j)(23 j-1)}{(n+1 n+21)(123)(234)},
\end{aligned}
$$

and

$$
\tilde{S}_{j}=(j-2 j-1 j)(j 12)(23 j-2), \quad j=6, \ldots, n+2 .
$$

The $F_{G r}^{k=3}(C)$ function is given by an essentially rearranged cyclic factor [26],

$$
F_{\mathrm{Gr}}^{k=3}(C)=\frac{1}{(123)(234) \cdots(n+212)} .
$$

Note that now this form of $F(C)$ corresponds to our choice (4.19).

As a side note let us point out that one can consider the $S_{j}$ or $\tilde{S}_{j}$ function as the explicit construction of the $\operatorname{map} \mathbf{S}=\left(\tilde{S}_{6}, \ldots, \tilde{S}_{n+2}\right), \mathbf{S}: \mathbb{C}^{(n-3)} \mapsto \mathbb{C}^{(n-3)}$, which zeros determine the integration contour ${ }^{23} \Gamma=\Gamma_{3, n+2}^{\text {tree }}$.

So the natural question is how can these different expressions $\left[F(C)\right.$ and $\left.F_{G r}(C)\right]$ provide us with the

\footnotetext{
${ }^{23}$ It is also important to mention that for $k>3$ analogs of $\left(\tilde{S}_{6}, \ldots, \tilde{S}_{n+2}\right)$ maps $\mathbf{S}: \mathbb{C}^{(k-2)(n-k)} \mapsto \mathbb{C}^{(k-2)(n-k)}$ may also be constructed $[103,105]$, and thus the explicit form of $\Gamma_{k, n+2}^{\text {tree }}$ integration contours is known.
}

representation of the same object? The answer to this question and also the resolution of our contradiction was given in [26,104]. It turns out that there actually exists a family of functions $F^{k=3}\left(C \mid t_{6}, \ldots, t_{n+2}\right)$ depending on parameters $t_{6}, \ldots, t_{n+2}$, such that

$$
\begin{aligned}
F^{k=3}\left(C \mid t_{6}, \ldots, t_{n+2}\right) & =H(C) \frac{1}{S_{6}\left(t_{6}\right) \cdots S_{n+2}\left(t_{n+2}\right)}, \\
H(C) & =\frac{\prod_{j=6}^{n+1}(12 j)(23 j-1) \prod_{i=5}^{n+1}(13 i)}{(n+1 n+21)(123)(345)},
\end{aligned}
$$

with $(j=6, \ldots, n+2)$

$$
\begin{aligned}
S_{j}(t)= & (j-2 j-1 j)(j 12)(23 j-2)(j-113) \\
& -t_{j}(j-1 j 1)(123)(3 j-2 j-1)(j 2 j-2),
\end{aligned}
$$

so that the result of evaluating by residues at zeros of the $\mathbf{S}(t)=\left(S_{6}\left(t_{6}\right), \ldots, S_{n+2}\left(t_{n+2}\right)\right)$ map the integral (5.5) is $t_{j}$ independent [26],

$$
\begin{aligned}
& \left.\partial_{t_{j}} \int_{\mathbf{S}(t)=0} d^{(n-k)} \tau_{A} F^{k=3}\left(C \mid t_{6}, \ldots, t_{n+2}\right)\right|_{c_{a i} \mapsto c_{a i}(\tau \mid \mathrm{kin})}=0 \\
& \quad \text { for } j=6, \ldots, n+2
\end{aligned}
$$

The case $t_{j}=0$ corresponds to the representation of the amplitude obtained from the Grassmannian integral representation, while the case $t_{j}=1$ corresponds to the representation obtained from scattering equation representation,

$$
\begin{aligned}
& F^{k=3}(C \mid 1, \ldots, 1)=F^{k=3}(C), \\
& \quad \text { and } F^{k=3}(C \mid 0, \ldots, 0)=F_{\mathrm{Gr}}^{k=3}(C) .
\end{aligned}
$$

The obtained relation thus supports the assertion that the Grassmannian integral representation has a stringy origin.

As an illustration let us consider the simplest case $k=3$, $n+2=6$. In this case we have integral over single complex parameter $\tau$ [it is assumed that in all minors the replacement $c_{a i} \mapsto c_{a i}(\tau \mid$ kin $)$ was performed],

$$
\begin{aligned}
A_{6,3} & =\int_{S(t)=0} d \tau \frac{(135)}{(123)(345)(561)} \frac{1}{S(t)}, \\
S(t) & =t(123)(345)(561)(246)-(234)(456)(612)(351),
\end{aligned}
$$

where minors (123), (345), (561), and $S(t)$ are the linear function of $\tau$. According to Cauchy theorem, the different residues are related with each other as

$$
\{S(t)\}=-\{(123)\}-\{(345)\}-\{(561)\}
$$


Here $\{\cdots\}$ denotes the integral residue at the corresponding pole. Note that for $(123)=0,(345)=0$, or $(561)=0$ the term in $S(t)$ proportional to $t$ vanishes, and as a consequence we have

$$
\partial_{t}\{(123)\}=\partial_{t}\{(345)\}=\partial_{t}\{(561)\}=0 .
$$

So, in the computation of the above integral we can put $S(t)$ to $S(0)$ and get

$$
\frac{(135)}{(123)(345)(561)} \frac{1}{S(0)}=\frac{1}{(123) \cdots(612)} .
$$

In the case $n+2>6$ the situation is similar, but now one must deal with multiple integrations over complex variables and use global residue theorem [26,104]. The explicit computations were also preformed for the $k=4$ case in [26], and it is believed that one can use the $F(C)$ function in the form of

$$
\begin{aligned}
F(C) & =F_{\mathrm{Gr}}(C) \\
& =\frac{1}{(1 \cdots k)(2 \cdots k+1) \cdots(n+2 \cdots k-1)}
\end{aligned}
$$

for general values of $n$ and $k$, which is why we also used the $F(C)$ function in the form of (4.19) in our considerations in the previous chapter. However, as far as we know there is no general proof of this assertion.

Let us once more stress that in all considerations above we never used the explicit form of kinematical dependence of [i.e., the explicit form of the solution of (5.4)] minors of matrix $C$. So the construction presented above will be valid not only for the $A_{k, n+2}$ amplitude but also for the integrand of (4.16) and in the analogous expression for the form factors of operators from the stress tensor supermultiplet, where, in both cases, some $\lambda$ 's and $\eta$ 's are rational functions of $\beta_{1,2}$ parameters. This is why we can replace $F(C)$ with $F_{G r}(C)$ according to (4.19).

At the end of this section, as an example, let us consider the simple nontrivial case of $n+2=6, k=3$ and check that we indeed get the same result independent of whether we faithfully use (5.2) or replace it with (5.21) as the $F(C)$ function in (4.16). Computing the string correlation function from the previous section for the $A_{3,4+1}^{*}$ amplitude we end up with the following expression [let us stress once again that in this formula $\lambda_{i}, \tilde{\lambda}_{i}, \tilde{\eta}_{i}$ for $i=5,6$ are functions of $\beta_{1,2}$ according to (4.7)]:

$$
\begin{aligned}
A_{3,4+1}^{*}= & \frac{\langle\xi p\rangle}{\kappa^{*}} \int \frac{\mathrm{d} \beta_{1} \wedge \mathrm{d} \beta_{2}}{\beta_{1} \beta_{2}} \frac{1}{\beta_{1}^{2} \beta_{2}} \frac{1}{\operatorname{VolGL}(3)} \\
& \times \int \mathrm{d}^{3 \times 6} C F(C) \delta^{k \times 2}(C \cdot \tilde{\lambda}) \\
& \times \delta^{3 \times 4}(C \cdot \tilde{\eta}) \delta^{(3) \times 2}\left(C^{\perp} \cdot \lambda\right),
\end{aligned}
$$

where $F(C)$ is given by (5.6) with $n=4$. Performing the change of variables (see also Appendix A) and evaluating the composite residue at points $\operatorname{res}_{\beta_{1}=-1}$ ores $_{\beta_{2}=0}$, we end up with

$$
\begin{aligned}
A_{3,4+1}^{*} & =\int_{S=0} d \tau \tilde{F}(C), \quad \tilde{F}(C)=\frac{(135)}{(123)(345)(561)} \frac{1}{S}, \\
S & =(123)(345)(561)(245)-(234)(456)(512)(351),
\end{aligned}
$$

and all minors, according to (5.4), are functions of external kinematical data defined as (4.25) with $n=4, k=3$. If we replace $F(C)$ with (5.12) according to our previous discussion, we will obtain

$$
\begin{aligned}
A_{3,4+1}^{*} & =\int_{\Gamma} d \tau \tilde{F}^{\prime}(C), \\
\text { where } \tilde{F}^{\prime}(C) & =\frac{(612)}{(512)} \frac{1}{(123) \cdots(612)},
\end{aligned}
$$

which is equivalent to (5.23) as expected, after an appropriate choice of $\Gamma$, which should encircle poles at (123), (345), and (561). Also from this example we see that in the case of $A_{3, n+1}^{*}$ off-shell amplitudes we can explicitly construct integration contours for their Grassmannian integral representations [i.e., the maps $\mathbf{S}=\left(\tilde{S}_{6}, \ldots, \tilde{S}_{n+2}\right)$, $\mathbf{S}: \mathbb{C}^{(n-3)} \mapsto \mathbb{C}^{(n-3)}$, whose zeros determine the integration contours $\Gamma_{3, n+2}^{\text {tree }}$ in (5.8)]. The latter are given by

$$
\begin{array}{rlr}
\tilde{S}_{j} & =(j-2 j-1 j)(j 12)(23 j-2), \quad j=6, \ldots, n+1, \\
\tilde{S}_{n+2} & =(n n+1 n+2)(n+112)(23 n) .
\end{array}
$$

This expression is easily obtained by considering integration contour $\left(\tilde{S}_{6}, \ldots, \tilde{S}_{n+2}\right)$ for the $n+2$ point on-shell amplitude and accounting for the $\operatorname{Reg} \sim(n+212) /$ $(n+112)$ factor. It is believed that in the case $k>3$ the integration contours can be constructed in a similar fashion.

At the end of this section let us make the following remark. Conditions (5.14) in general and for $n+2=6$ in particular can be interpreted as conditions for six points that lie on a conic. For example,

$$
\begin{aligned}
S(1)= & (123)(345)(561)(246) \\
& -(234)(456)(612)(351)=0
\end{aligned}
$$

is the condition that six points in Grassmannian $\mathbb{C P}^{2}$ lie on a single conic (any general five points determines a conic, so this is a condition that point 6 also belongs to the conic). It is natural to ask if there is any geometrical interpretation for

$$
S=(123)(345)(561)(245)-(234)(456)(512)(351)=0,
$$


which appears in our construction. We found the conditions on matrix $C$ to be of Veronese form, $S=0$ are equivalent to $\sigma_{6}=\sigma_{5}$, and $\sigma_{1}, \ldots, \sigma_{5}$ are arbitrary (here as in [26] we rescaled all $s_{i}$ to 1 ); i.e., we interpret this condition as point 6 belongs to the conic and coincides with point 5 . This is probably not very surprising. The dimensionality of the Grassmannian is related to the number of independent kinematical variables. In the case of off-shell amplitudes (Wilson line form factors) we use axillary spinors $\lambda_{p}$ and $\lambda_{\xi}$ in the description of off-shell momenta. But in the final result dependence on $\lambda_{\xi}$ drops out $[53,63,64]$, so effectively we have fewer variables than naively expected.

\section{GLUING PROCEDURE AND AMPLITUDES}

Let us return once again to the formula (4.16). We have seen in the previous sections that if we leave integrals with respect to $\beta_{1,2}$ intact and concentrate on integrations with respect to $\mathrm{d} s_{a} \mathrm{~d} \sigma_{a}$, the result (after an appropriate choice of integration contours) will be proportional to the $A_{k, n+2}$ on-shell amplitude where the dependence on $\beta_{1,2}$ is condensed in kinematical variables $\left\{\lambda_{i}, \tilde{\lambda}_{i}, \eta_{i}\right\}_{i=n+1}^{n+2}$ [see (4.7) and (4.9)].

So we can think of some integral operator $\hat{A}$ which directly transforms on-shell amplitudes into Wilson line form factors and correlation functions:

$$
\hat{A}: A_{k, n+2} \mapsto A_{k, n+1}^{*} .
$$

We will call this operator the gluing operator and will label it $\hat{A}_{i, i+1}$. Label $i$ corresponds to the position of the kinematical variables on which this operator acts. Another way to introduce this operator is simply to consider convolution (in the seance of discussion at the beginning of Sec. IV) of the $A_{2,2+1}^{*}$ minimal off-shell amplitude with some function of $\left\{\lambda_{i}, \tilde{\lambda}_{i}, \eta_{i}\right\}$. In this sense the gluing operation (for Wilson line form factors and correlation functions) discussed in Sec. IV is given by the action of the gluing operator.

From a practical point of view it is useful because one can immediately utilize a large library of answers for on-shell amplitudes into Wilson line form factors and correlation functions, which in turn can be interpreted as Reggeon amplitudes.

Note also that a similar procedure should work for the form factors of operators from the stress tensor supermultiplet, i.e., for different choices of the $\psi$ target space wave function, which participates in the gluing procedure, though we will not discuss it in detail. We leave this topic for a separate publication. Here we will concentrate on the simplest case of Wilson line form factors and correlation functions.

So more formally let us define gluing operator $\hat{A}_{n+1, n+2}[\cdots]$ acting on the space of functions $f$ of $\left\{\lambda_{i}, \tilde{\lambda}_{i}, \eta_{i}\right\}_{i=1}^{n+2}$ variables as

$$
\begin{aligned}
\hat{A}_{n+1, n+2}[f] \equiv & \int \prod_{i=n+1}^{n+2} \frac{d^{2} \lambda_{i} d^{2} \tilde{\lambda}_{i} d^{4} \eta}{\operatorname{Vol}[G L(1)]} A_{2,2+1}^{*} \\
& \times f\left(\left\{\lambda_{i}, \tilde{\lambda}_{i}, \eta_{i}\right\}_{i=1}^{n+2}\right) .
\end{aligned}
$$

Performing integration over $\tilde{\lambda}_{n+1}, \tilde{\lambda}_{n+2}, \tilde{\eta}_{n+1}$, and $\tilde{\eta}_{n+2}$ variables as in Appendix A we get

$\hat{A}_{n+1, n+2}[f]=\left.\frac{\langle p \xi\rangle}{\kappa^{*}} \int \frac{d \beta_{1}}{\beta_{1}} \wedge \frac{d \beta_{2}}{\beta_{2}} \frac{1}{\beta_{1}^{2} \beta_{2}} f\left(\left\{\lambda_{i}, \tilde{\lambda}_{i}, \tilde{\eta}_{i}\right\}_{i=1}^{n+2}\right)\right|_{*}$,

where $\left.\right|_{*} \quad$ denotes substitutions $\quad\left\{\lambda_{i}, \tilde{\lambda}_{i}, \eta_{i}\right\}_{i=n+1}^{n+2} \mapsto$ $\left\{\lambda_{i}(\beta), \tilde{\lambda}_{i}(\beta), \tilde{\eta}_{i}(\beta)\right\}_{i=n+1}^{n+2}$ with

$\lambda_{n+1}(\beta)=\underline{\lambda}_{n+1}+\beta_{2} \underline{\lambda}_{n+2}$,

$\tilde{\lambda}_{n+1}(\beta)=\beta_{1} \tilde{\lambda}_{n+1}+\frac{\left(1+\beta_{1}\right)}{\beta_{2}} \tilde{\lambda}_{n+2}$,

$\tilde{\eta}_{n+1}(\beta)=-\beta_{1} \underline{\underline{\eta}}_{n+1}^{\tilde{\tilde{n}}}, \quad \lambda_{n+2}(\beta)=\underline{\underline{\lambda}}_{n+2}+\frac{\left(1+\beta_{1}\right)}{\beta_{1} \beta_{2}} \underline{\lambda}_{n+1}$,

$\tilde{\lambda}_{n+2}(\beta)=-\beta_{1} \underline{\tilde{\lambda}}_{n+2}-\beta_{1} \beta_{2} \underline{\tilde{\lambda}}_{n+1}, \quad \tilde{\eta}_{n+2}(\beta)=\beta_{1} \beta_{2} \underline{\underline{\eta}}_{n+1}$,

and

$\underline{\underline{\lambda}}_{n+1}=\lambda_{p}, \quad \underline{\tilde{\lambda}}_{n+1}=\frac{\langle\xi| k}{\langle\xi p\rangle}, \quad \underline{\tilde{\eta}}_{n}=\tilde{\eta}_{p}$

$\underline{\underline{\lambda}}_{n+2}=\lambda_{\xi}, \quad \underline{\tilde{\lambda}}_{n+2}=\frac{\langle p| k}{\langle\xi p\rangle}, \quad \underline{\tilde{\eta}}_{n+2}=0$.

Here we also understand integration with respect to $\beta_{1,2}$ as a residue form, and we will always evaluate it at points $\operatorname{res}_{\beta_{2}=0} \circ \mathrm{res}_{\beta_{1}=-1}$. After this formal introduction we are ready to consider several examples of action of $\hat{A}_{i, i+1}$ on on-shell amplitudes.

\section{A. Tree level}

So let us test how our gluing operator works on some explicit examples. The simplest case is given by the action of $\hat{A}$ on $k=2, n+2=4$ point amplitude $A_{2,4}$. We expect that we will reproduce the $A_{2,2+1}^{*}$ off-shell amplitude (Wilson line operator form factor) by the action of operator $\hat{A}_{34}[\cdots]$ on the $A_{2,4}$ on-shell amplitude. Note also that the next steps are actually identical for all $k=2$ amplitudes with arbitrary $n$. Indeed, in the case of the $A_{2,4}$ amplitude we have

$$
A_{2,4}\left(\Omega_{1}, \ldots, \Omega_{4}\right)=\delta^{4}\left(p_{1234}\right) \frac{\delta^{8}\left(q_{1234}\right)}{\langle 12\rangle\langle 23\rangle\langle 34\rangle\langle 41\rangle} .
$$


Introducing notations ${ }^{24}$

$$
p_{1 \cdots n} \equiv \sum_{i=1}^{n} p_{i} \equiv \sum_{i=1}^{n} \lambda_{i} \tilde{\lambda}_{i}, \quad p_{1 \cdots n}^{2}=p_{1, n}^{2}, \quad q_{1 \cdots n} \equiv \sum_{i=1}^{n} \lambda_{i} \tilde{\eta}_{i}
$$

the $A_{2,4}$ amplitude with $\left.\right|_{*}$ substitution applied takes the form

$$
\left.A_{2,4}\right|_{*}=\delta^{4}\left(p_{12}+k\right) \frac{\delta^{8}\left(q_{12 p}\right) \beta_{1}^{2} \beta_{2}}{\langle 12\rangle\left(\langle 2 p\rangle+\beta_{2}\langle 2 \xi\rangle\right)\langle p \xi\rangle\left(\beta_{1} \beta_{2}\langle 1 \xi\rangle+\left(1+\beta_{1}\right)\langle 1 p\rangle\right)} .
$$

Now, evaluating the integral over $\beta_{1}, \beta_{2}$ by means of composite residue $\operatorname{res}_{\beta_{1}=-1}$ ores $_{\beta_{2}=0}[\cdots]$ we get

$$
\hat{A}_{34}\left[A_{2,4}\left(\Omega_{1}, \ldots, \Omega_{4}\right)\right]=\delta^{4}\left(p_{12}+k\right) \frac{\delta^{8}\left(q_{12 p}\right)}{\kappa^{*}\langle 12\rangle} \operatorname{res}_{\beta_{2}=0} \circ \operatorname{res}_{\beta_{1}=-1}[\omega]=A_{2,2+1}^{*}\left(\Omega_{1}, \Omega_{2}, g_{3}^{*}\right),
$$

where

$$
\omega=\frac{d \beta_{2} \wedge d \beta_{1}}{\beta_{2} \beta_{1}\left(\langle 2 p\rangle+\beta_{2}\langle 2 \xi\rangle\right)\left(\beta_{1} \beta_{2}\langle 1 \xi\rangle+\left(1+\beta_{1}\right)\langle 1 p\rangle\right)},
$$

and (the projector $\partial_{\eta_{p}}^{4}$ acting on $A_{2, n+1}^{*}$ is implemented)

$$
A_{2,2+1}^{*}\left(\Omega_{1}, \Omega_{2}, g_{3}^{*}\right)=\delta^{4}\left(p_{12}+k\right) \frac{1}{\kappa^{*}} \frac{\delta^{8}\left(q_{12 p}\right)}{\langle p 1\rangle\langle 12\rangle\langle 2 p\rangle} .
$$

This is in agreement with results of $[53,63]$ for $A_{2,2+1}^{*}$.

Proceeding in a similar way let us consider the action of $\hat{A}_{n+1 n+2}$ on the $A_{2, n+2}$ on-shell amplitude. We expect that the result will be given by the $A_{2, n+1}^{*}$ Wilson line form factor. Indeed, it is easy ${ }^{25}$ to see that

$$
\hat{A}_{n+1 n+2}\left[A_{2, n+2}\left(\Omega_{1}, \ldots, \Omega_{n+2}\right)\right]=A_{2, n+1}^{*}\left(\Omega_{1}, \ldots, \Omega_{n}, g_{n+1}^{*}\right)=\frac{\delta^{4}\left(p_{1 \cdots n}+k\right)}{\kappa^{*}} \frac{\delta^{8}\left(q_{1 \cdots p}\right)}{\langle p 1\rangle\langle 12\rangle \cdots\langle n p\rangle}
$$

This is once more in agreement with the results of $[53,63]$ for $A_{2, n+1}^{*}$.

Proceeding further in a similar way we can also reproduce other results obtained from BCFW recursion $[53,63]$ for component off-shell amplitudes (Wilson line operator form factors) $A_{3,3+1}^{*}\left(1^{-} 2^{-} 3^{+} g_{4}^{*}\right)$ and $A_{3,4+1}^{*}\left(1^{+} 2^{+} 3^{-} 4^{-} g_{5}^{*}\right)$ $[53,63]$. We expect to obtain them from the on-shell amplitudes $A_{3,5}\left(1^{-} 2^{-} 3^{+} 4^{-} 5^{+}\right)$and $A_{3,6}\left(1^{+} 2^{+} 3^{-} 4^{-} 5^{-} 6^{+}\right)$ by means of the action of $\hat{A}_{45}[\cdots]$ and $\hat{A}_{56}[\cdots]$ correspondingly. Performing simple computations (the explicit details and answers can be found in Appendix B) we see that indeed the following relations holds:

$$
\hat{A}_{45}\left[A_{3,5}\left(1^{-} 2^{-} 3^{+} 4^{-} 5^{+}\right)\right]=A_{3,3+1}^{*}\left(1^{-} 2^{-} 3^{+} g_{4}^{*}\right),
$$

\footnotetext{
${ }^{24}$ Here for simplicity we also drop spinorial and $S U(4)_{R}$ indices.

${ }^{25}$ It can be obtained by simple spinor relabelings from a previous example.
}

$$
\hat{A}_{56}\left[A_{3,6}\left(1^{+} 2^{+} 3^{-} 4^{-} 5^{-} 6^{+}\right)\right]=A_{3,4+1}^{*}\left(1^{+} 2^{+} 3^{-} 4^{-} g_{5}^{*}\right),
$$

in agreement with previously obtained results for Wilson line form factors $[53,63]$.

As a final example we would like to consider the case with multiple gluing operations applied. Let us consider a quite nontrivial example of such a situation; namely, let us consider the correlation function of three Wilson line operators $A_{3,0+3}^{*}\left(g_{1}^{*}, g_{2}^{*}, g_{3}^{*}\right)$. According to our construction of generalized world-sheet vertex operators, it should be given by

$$
A_{3,0+3}^{*}\left(g_{1}^{*}, g_{2}^{*}, g_{3}^{*}\right)=\left\langle\mathcal{V}_{1,2}^{\mathrm{WL}} \mathcal{V}_{3,4}^{\mathrm{WL}} \mathcal{V}_{5,6}^{\mathrm{WL}}\right\rangle
$$

It was first computed in [53] by means of BCFW recursion and later reproduced in [64] from Grassmannian integral representation. The result is given by 


$$
\begin{aligned}
A_{3,0+3}^{*}\left(g_{1}^{*}, g_{2}^{*}, g_{3}^{*}\right) & =\delta^{4}\left(k_{1}+k_{2}+k_{3}\right)\left(1+\mathbb{P}^{\prime}+\mathbb{P}^{\prime 2}\right) \tilde{f}, \\
\tilde{f} & =\frac{\left\langle p_{1} p_{2}\right\rangle^{3}\left[p_{2} p_{3}\right]^{3}}{\kappa_{3} \kappa_{1}^{*}\left\langle p_{2}\left|k_{1}\right| p_{3}\right]\left\langle p_{1}\left|k_{3}\right| p_{2}\right]\left\langle p_{2}\left|k_{1}\right| p_{2}\right]} .
\end{aligned}
$$

Here $\mathbb{P}^{\prime}$ is the permutation operator that now shifts all spinor and momenta labels by $+1 \bmod 3$.

We want to show that $A_{3,0+3}^{*}$ can be reproduced from the $\mathrm{NMHV}_{6}$ point on-shell amplitude $A_{3,6}\left(1^{-} 2^{+} 3^{-} 4^{+} 5^{-} 6^{+}\right)$by application of the following product of gluing operators $\hat{A}_{12} \circ \hat{A}_{34} \circ \hat{A}_{56}$. Indeed (see Appendix B for details), the following relation holds:

$\left.A_{3,0+3}^{*}\left(g_{1}^{*}, g_{2}^{*}, g_{3}^{*}\right)=\left(\hat{A}_{12} \circ \hat{A}_{34} \circ \hat{A}_{56}\right)\left[A_{3,6}\left(1^{-} 2^{+} 3^{-} 4^{+} 5^{-} 6^{+}\right)\right]\right]$,

where the $A_{3,6}\left(1^{-} 2^{+} 3^{-} 4^{+} 5^{-} 6^{+}\right)$amplitude is given by

$$
\begin{aligned}
A_{3,6} & =\delta^{4}\left(p_{1 \ldots 6}\right)\left(1+\mathbb{P}^{2}+\mathbb{P}^{4}\right) f, \\
f & =\frac{\langle 13\rangle^{4}[46]^{4}}{\langle 12\rangle\langle 23\rangle[45][56]\langle 3|1+2| 6]\langle 1|5+6| 4] p_{456}^{2}},
\end{aligned}
$$

and $\mathbb{P}$ is the permutation operator shifting spinor labels by $+1 \bmod 6$.

So we have seen in multiple examples that are presented at the beginning of this section that gluing operator $\hat{A}_{i, i+1}$ allows one to convert on-shell amplitudes into Wilson line form factors and correlation functions at tree level.

At the end of this subsection we would like to point out the formal analogy between the action of $\hat{A}_{i i+1}$ operators on $A_{k, n}$ amplitudes and the action of $R$ matrices on some vacuum state of integrable spin chain. Indeed, it looks like the $\hat{A}_{i i+1}$ operator creates "excitation" (Wilson line operator insertion) in the "vacuum" consisting from on-shell states. We think that this analogy being properly investigated may provide us with the answer to the question "what is the appropriate description of Wilson line form factors in terms of some integrable system?"

It is also interesting to note that the integration with respect to $\beta_{1}, \beta_{2}$ variables, which was performed by taking residues, is, in fact, equivalent to the choice of a specific kinematical limit for the momenta $p_{n+1}$ and $p_{n+2}$ of the initial on-shell amplitude. If we naively take consecutive limits $\beta_{1} \rightarrow-1, \beta_{2} \rightarrow 0$ in the definitions of momenta $p_{n+1}(\beta)$ and $p_{n+2}(\beta)$, which is equivalent to residue evaluation, we would get the finite result ${ }^{26}$

$$
p_{n+1}=\underline{\underline{\lambda}}_{n+1} \stackrel{\tilde{\lambda}}{=}_{n+1}, \quad p_{n+2}=\underline{\underline{\lambda}}_{n+2} \stackrel{\tilde{\lambda}}{=}_{n+2} .
$$

\footnotetext{
${ }^{26}$ Note that if we would take the limits in opposite order, the results for $p_{n+1}, p_{n+2}$ momenta would diverge, but the expression for off-shell amplitudes would still be finite.
}

On the other hand, for all $\omega$ forms that we have encountered in previous examples we may use the global residue theorem to relate multiple residues at $\beta_{1}=-1, \beta_{2}=0$ with the multiple residues at $\beta_{1}=0, \beta_{2}=0$. If we take the limits $\beta_{1} \rightarrow 0, \beta_{2} \rightarrow 0$ (regardless of the order of limits) in the definitions of $p_{n+1}(\beta)$ and $p_{n+2}(\beta)$ momenta, we will get the singular result

$$
\begin{aligned}
& p_{n+1}=\frac{1}{\beta_{2}} \underline{\lambda}_{n+1} \tilde{\tilde{\lambda}}_{n+2}+\underline{\underline{\lambda}}_{n++2} \stackrel{\tilde{\lambda}}{=}_{n+2}+O\left(\beta_{2}\right), \\
& p_{n+2}=\frac{1}{\beta_{2}} \underline{\lambda}_{n+1} \underline{\tilde{\lambda}}_{n+2}+\underline{\underline{\lambda}}_{n+1} \tilde{\tilde{\lambda}}_{n+1}+O\left(\beta_{2}\right),
\end{aligned}
$$

which is equivalent to BCFW shift $[n+1, n+2\rangle$ of $p_{n+1}=\underline{\underline{\lambda}}_{n+2} \stackrel{\tilde{\lambda}}{\underline{n}+2}_{n+2}$ and $p_{n+2}=\underline{\underline{\lambda}}_{n+1} \stackrel{\tilde{\lambda}}{\underline{\lambda}+1}_{n+1}$ momenta evaluated at large $z$. The behavior of amplitudes in the limit $z \rightarrow \infty$ may be interpreted as a special kinematical limit with some particles with large (complex) lightlike momenta traveling in the soft background [106]. So in this sense our gluing procedure is closely related to the specific high energy kinematical limit of the ordinary on-shell amplitudes.

\section{B. Integrands}

So far we have seen that by means of gluing operator $\hat{A}_{i, i+1}$ we can convert on-shell amplitudes into Wilson line form factors and correlation functions at tree level, formally without any reference to ambitwistor string or Grassmannian representation, though the explicit form of this operator is, of course, motivated heavily by our construction of generalized vertex operators.

But what about loops? Since our gluing operator acts in a simple manner on rational functions, it is natural to try to verify conjecture that using $\hat{A}_{i, i+1}$ we can convert integrands of on-shell amplitudes into integrands of Wilson line form factors and correlation functions as well.

To see whether this conjecture is reasonable, let us consider the simplest possible example of $k=2, n+1=3$ point one loop amplitude $A_{2,2+1}^{*(1)}$ and show that the gluing operator applied to the integrand of the $A_{2,4}^{(1)}$ amplitude will give us the desired expression for the integrand of the $A_{2,2+1}^{*(1)}$ amplitude.

But first we need to obtain the integrand of the $A_{2,2+1}^{*(1)}$ amplitude itself. The easiest way to get it is to try to reconstruct it from the $k^{2}$ channel unitarity cut. Considering the latter (taking residues of the integrand with respect to the poles of $1 / l_{1}^{2}$ and $1 / l_{2}^{2}$ propagators; see Fig. 1) we have $^{27}$

\footnotetext{
${ }^{27}$ The necessary manipulations are similar to the case of the $s$ channel cut of the $A_{2,4}^{(1)}$ amplitude. $(p q)$ here stands for standard Mincovskian scalar product.
} 


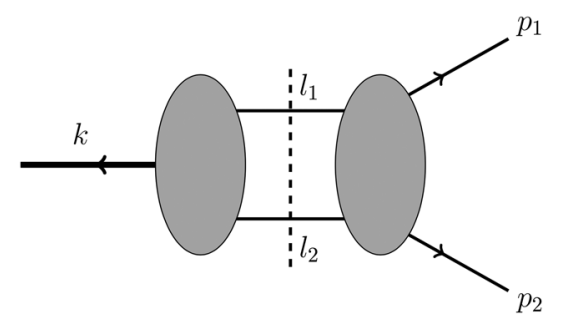

FIG. 1. Unitarity cut of the $A_{2,2+1}^{*(1)}$ amplitude in the $k^{2}=$ $\left(p_{1}+p_{2}\right)^{2}$ channel.

$$
\begin{aligned}
\left.A_{2,2+1}^{*(1)}\right|_{k^{2} \text { cut }} & =\int d^{4} \eta_{l_{1}} d^{4} \eta_{l_{2}} A_{2,2+1}^{*}\left(l_{1}, l_{2}, g^{*}\right) A_{2,4}\left(l_{1}, l_{2}, \Omega_{2}, \Omega_{1}\right) \\
& =A_{2,2+1}^{*}\left(l_{1}, l_{2}, g^{*}\right) \frac{\operatorname{Tr}(k p 21)}{\left(p l_{2}\right)\left(l_{2} 2\right)}
\end{aligned}
$$

The Tr factor can be transformed into $k^{2}\left(p+p_{2}\right)^{2}=$ $\left(p_{1}+p_{2}\right)^{2}\left(p_{2}+p\right)^{2}$ with the help of momentum conservation $k+p_{1}+p_{2}=0$, and $k_{T}$ decomposition conditions $(p k)=0$. Thus, the expression for the $A_{2,2+1}^{*(1)}\left(\Omega_{1}, \Omega_{2}, g^{*}\right)$ amplitude is given by

$$
\begin{aligned}
A_{2,2+1}^{*(1)}\left(\Omega_{1}, \Omega_{2}, g^{*}\right)= & A_{2,2+1}^{*}\left(\Omega_{1}, \Omega_{2}, g^{*}\right) \int d^{D} l \\
& \times \frac{\left(p_{1}+p_{2}\right)^{2}\left(p_{2}+p\right)^{2}}{l^{2}\left(l+p_{2}\right)^{2}\left(l+p_{1}+p_{2}\right)^{2}(p l)},
\end{aligned}
$$

which contains one loop scalar box integral with one of the propagators, namely, $1 / l^{2}$ replaced by its eikonal counterpart $1 /(p l)$; see Fig. 2.

Now let us turn to the integrand of the $A_{2,4}^{(1)}$ amplitude:

$$
\begin{aligned}
A_{2,4}^{(1)}\left(\Omega_{1}, \ldots, \Omega_{4}\right)= & A_{2,4}\left(\Omega_{1}, \ldots, \Omega_{4}\right) \int d^{D} l \\
& \times \frac{\left(p_{1}+p_{2}\right)^{2}\left(p_{2}+p_{3}\right)^{2}}{l^{2}\left(l+p_{2}\right)^{2}\left(l+p_{1}+p_{2}\right)^{2}\left(l-p_{3}\right)^{2}} .
\end{aligned}
$$

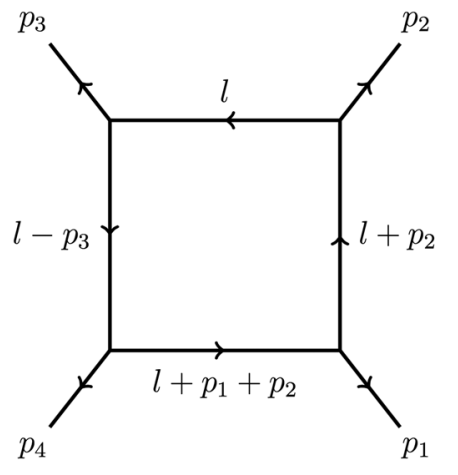

(a)

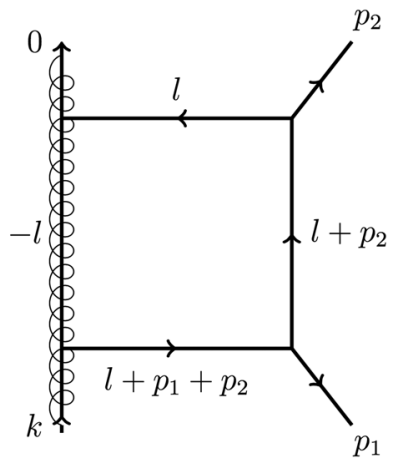

(b)
FIG. 2. Scalar box integrals contributing to $A_{2,2+1}^{(1)}$ and $A_{2,2+1}^{*(1)}$ amplitudes correspondingly. The line with the coil denotes eikonal propagator $1 /(p l)$.
It should be noted that the notion of the integrand is uniquely defined only in dual variables. So, to be accurate we should consider the gluing operation in such variables (momentum twistors) also. Here however, we will use helicity spinors in a hope that possible loop momentum rearrangement will not cause any trouble. It turns out that it is indeed the case as we will see in a moment. Acting with the $\hat{A}_{34}$ operator on the $A_{2,4}^{(1)}(1,2,3,4)$ integrand

$$
\begin{aligned}
\text { Int }= & A_{2,4}\left(\Omega_{1}, \ldots, \Omega_{4}\right) \\
& \times \frac{\left(p_{1}+p_{2}\right)^{2}}{l^{2}\left(l+p_{2}\right)^{2}\left(l+p_{1}+p_{2}\right)^{2}} \frac{\left(p_{2}+p_{3}\right)^{2}}{\left(l-p_{3}\right)^{2}},
\end{aligned}
$$

and using momentum definitions (B5) we get

$$
\begin{aligned}
\hat{A}_{34}[\operatorname{Int}]= & A_{2,2+1}^{*}\left(\Omega_{1}, \Omega_{2}, g_{3}^{*}\right) \\
& \times \frac{\left(p_{1}+p_{2}\right)^{2}}{l^{2}\left(l+p_{2}\right)^{2}\left(l+p_{1}+p_{2}\right)^{2}} \frac{\left(p_{2}+p\right)^{2}}{(l p)},
\end{aligned}
$$

which is exactly the integrand expression for the $A_{2,2+1}^{*(1)}$ amplitude.

This example gives us hope that a more accurate and general consideration of the gluing procedure at the level of integrands will also be successful and will provide us with the prescription for obtaining $A_{k, m+n}^{*(l)}$ integrands from the corresponding $A_{k, m+2 n}^{(l)}$ integrands by the application of appropriate combinations of $\hat{A}_{i i+1}$ operators.

\section{CONCLUSION}

In this paper we presented results for the derivation of scattering equations (RSV) and Grassmannian representations for form factors of local and Wilson line operators in $\mathcal{N}=4 \mathrm{SYM}$ from corresponding four-dimensional ambitwistor string theory. In the case of local operators we restricted ourselves to the case of form factors of operators from stress-tensor operator supermultiplet. The obtained results are in agreement with previously obtained Grassmannian integral representations. As a byproduct we discovered an easy and convenient gluing procedure, which allows us to obtain the required form factor expressions from already known amplitude expressions. The construction of composite string vertex operators for the analyzed local or Wilson line operators was inspired by the mentioned gluing procedure. An interesting future research direction, which we are planning to pursue, will be to consider pullbacks of composite operators defined on twistor or Lorentz harmonic chiral superspace [77-83]. We hope that our consideration along these lines could be extended to arbitrary local composite operators.

Next, it would be very interesting to fully uncover the geometrical picture behind Grassmannian and scattering 
equation representations for form factors of local and Wilson line operators. It is interesting to see if the amplituhedron picture could be extended to all possible gauge invariant observables in $\mathcal{N}=4 \mathrm{SYM}$, for which representations under global gauge transformations may differ from singlet representation.

Having obtained scattering equation representations, one may wonder what is the most efficient way to get final expressions for particular form factors with a given number of particles and their helicities. In the case of amplitudes we know that it is given by computation of global residues with the methods of computational algebraic geometry [107109]; see also [105]. It would be interesting to see how this procedure works in the case of tree level form factors and their loop level integrands, and to provide necessary details needed when writing computer codes.

Finally, it would be interesting to consider loop corrections to form factors of Wilson line operators (gauge invariant offshell amplitudes). Also, it is extremely interesting to see how the presented approach works in other theories, for example, in gravity and supergravity (see recent discussion [110] of super gravity (SUGRA) amplitudes in a similar context considered here), where in the case of gauge invariant offshell amplitudes we have a well developed framework based on a high-energy effective Lagrangian [111-115]; see also [116-120] for similar research along this direction.

\section{ACKNOWLEDGMENTS}

The authors thank D. I. Kazakov, L. N. Lipatov, and Yu-tin Huang for interesting and stimulating discussions. This work was supported by RFBR Grants No. 17-02-00872 and No. 16-02-00943 and Contract No. 02.A03.21.0003 from 27.08.2013 with Russian Ministry of Science and Education. The work of L. V. B. was supported by Grant No. 17-13-325-1 of the Basis foundation for theoretical physics.

\section{APPENDIX A: GLUING PROCEDURE AND GRASSMANNIANS}

In this appendix we are going to present computational details from Sec. IV of the main text.

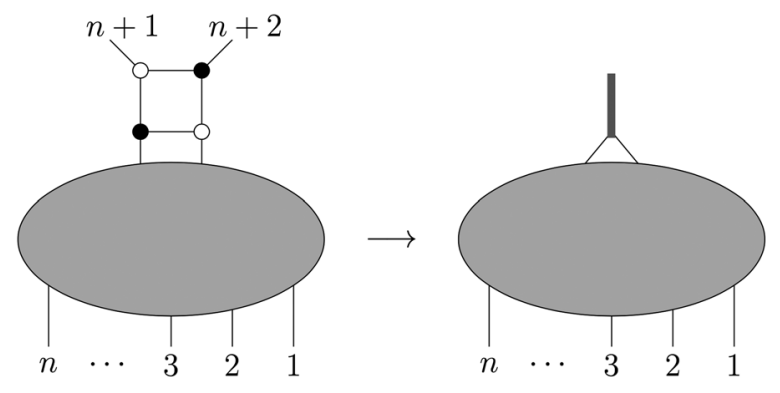

FIG. 3. Gluing procedure.

Before proceeding with actual formulas, let us make the following remark. The gluing procedure (gluing operator) introduced in [74] was, in fact, implicitly used to obtain both the Grassmannian integral representation for form factors of operators from the stress tensor supermultiplet [74] and off-shell amplitudes with one leg off-shell [63]. The idea was to take a top-cell diagram for amplitude, perform a sequence of square and merge/unmerge moves until we get a box (assume it is possible for the diagram under consideration) on the boundary, and replace it with the corresponding minimal form factor or off-shell amplitude. Graphically, this relation reads ${ }^{28}$ Fig. 3 where the box at the legs $n+1$ and $n+2$ was replaced for the sake of concreteness. We got a similar relation of form factor onshell diagrams to the amplitude on-shell diagrams in $[73,76]$ based on the soft limit procedure. The corresponding box diagram was deformed by an extra soft factor, so that it became equivalent to the corresponding minimal form factor.

It turns out, however, that there is a simpler gluing procedure, which we used in our consideration. Namely, we can glue (perform the on-shell phase space integrationperform convolution with) minimal form factor or off-shell amplitude directly to the amplitude top cell diagram without cutting off the boxes mentioned above.

Now let us return to actual computations. Let us consider the case of Wilson line operators first. Let us consider (4.11) once more. If we choose $F(C)$ according to (4.17), we can rewrite $(4.11)$ as $^{29}$

$$
A_{k, n+1}^{*}=\left.\int \prod_{i=n+1}^{n+2} \frac{d^{2} \lambda_{i} d^{2} \tilde{\lambda}_{i}}{\operatorname{Vol}[G L(1)]} d^{4} \tilde{\eta}_{i} A_{2,2+1}^{*}\left(g^{*}, \Omega_{n+1}, \Omega_{n+2}\right)\right|_{\lambda \rightarrow-\lambda} A_{k, n+2},
$$

where $A_{k, n+2}$ stands for the corresponding Grassmannian integral-top-cell diagram (which can be evaluated into the $\mathrm{N}^{k-2} \mathrm{MHV} n+2$ point on-shell amplitude if the appropriate integration contour is chosen), ${ }^{30}$

\footnotetext{
${ }^{28}$ We have borrowed this nice picture from [74].

${ }^{29}$ Without loss of generality we may choose the off-shell leg to lie between legs 1 and $n$.

${ }^{30}$ Here we left the integration contour unspecified.
} 


$$
A_{k, n+2}=\int \frac{d^{k \times(n+2)} C}{\operatorname{Vol}[G L(k)]} \frac{\delta^{k \times 2}(C \cdot \tilde{\lambda}) \delta^{k \times 4}(C \cdot \tilde{\eta}) \delta^{(n+2-k) \times 2}\left(C^{\perp} \cdot \lambda\right)}{(1 \cdots k)(2 \cdots k+1) \cdots(n+2 \cdots k-1)},
$$

and the minimal off-shell vertex $A_{2,2+1}^{*}\left(g^{*}, n+1, n+2\right)$ is given by [63]

$$
\begin{aligned}
A_{2,2+1}^{*}\left(g^{*}, \Omega_{n+1}, \Omega_{n+2}\right)= & \frac{1}{\kappa^{*}} \int \frac{d^{2 \times 3} C}{\operatorname{Vol}[G L(2)]} \frac{\delta^{4}(C \cdot \underline{\tilde{\lambda}}) \delta^{8}(C \cdot \tilde{\eta}) \delta^{4}\left(C^{\perp} \cdot \lambda\right)}{(p n+1)(n+1 n+2)(n+2 p)} \\
= & \frac{1}{\kappa^{*}} \int \frac{\mathrm{d} \beta_{1}}{\beta_{1}} \frac{\mathrm{d} \beta_{2}}{\beta_{2}} \delta^{2}\left(\lambda_{p}+\beta_{1} \lambda_{n+1}-\beta_{1} \beta_{2} \lambda_{n+2}\right) \delta^{2}\left(\underline{\tilde{\lambda}}_{n+1}\right) \delta^{2}\left(\tilde{\tilde{\lambda}}_{n+2}\right) \\
& \times \delta^{4}\left(\tilde{\eta}_{n+1}+\beta_{1} \tilde{\eta}_{p}\right) \delta^{4}\left(\tilde{\eta}_{n+2}+\beta_{2} \tilde{\eta}_{n+1}\right)
\end{aligned}
$$

with $\tilde{\lambda}_{n+1}=\tilde{\lambda}_{n+1}+\frac{\langle n+2| k}{\langle n+2 \mid n+1\rangle}$ and $\tilde{\lambda}_{n+2}=\tilde{\lambda}_{n+2}+\frac{\langle n+1| k}{\langle n+1 \mid n+2\rangle}$. Here $p$ is the off-shell gluon direction and $k$ is its momentum. Now, the integration steps up to final integrations in $\beta_{1}$ and $\beta_{2}$ follow closely those in [63]. That is, performing integrations over $\tilde{\lambda}_{n+1}, \tilde{\lambda}_{n+2}, \tilde{\eta}_{n+1}$, and $\tilde{\eta}_{n+2}$, we get

$$
\begin{array}{cl}
\tilde{\lambda}_{n+1}=-\frac{\langle n+2| k}{\langle n+2 \mid n+1\rangle}, & \tilde{\lambda}_{n+2}=-\frac{\langle n+1| k}{\langle n+1 \mid n+2\rangle}, \\
\tilde{\eta}_{n+1}=-\beta_{1} \tilde{\eta}_{p}, \quad \tilde{\eta}_{n+2}=\beta_{1} \beta_{2} \tilde{\eta}_{p} .
\end{array}
$$

The $\operatorname{Vol}[G L(1)]^{2}$ redundancy in the remaining integrations over $\lambda$ is removed using their parametrization as in [74]

$$
\lambda_{n+1}=\xi_{A}-\beta_{3} \xi_{B}, \quad \lambda_{n+2}=\xi_{B}-\beta_{4} \xi_{A},
$$

where $\xi_{A}$ and $\xi_{B}$ are two arbitrary but linearly independent reference spinors. Then $\langle n+1 n+2\rangle=\left(\beta_{3} \beta_{4}-1\right)\left\langle\xi_{B} \xi_{A}\right\rangle$,

$$
\int \frac{d^{2} \lambda_{n+1}}{\operatorname{Vol}[G L(1)]} \frac{d^{2} \lambda_{n+2}}{\operatorname{Vol}[G L(1)]}=-\left\langle\xi_{A} \xi_{B}\right\rangle^{2} \int d \beta_{3} d \beta_{4},
$$

and

$$
\begin{aligned}
A_{k, n+1}^{*}= & \frac{1}{\kappa^{*}}\left\langle\xi_{A} \xi_{B}\right\rangle^{2} \int \frac{d^{k \times(n+2)} C}{\operatorname{Vol}[G L(k)]} \frac{d \beta_{1}}{\beta_{1}} \frac{d \beta_{2}}{\beta_{2}} \frac{d \beta_{3} d \beta_{4}}{\left(1-\beta_{3} \beta_{4}\right)^{2}} \\
& \times \delta^{2}\left(\lambda_{p}+\beta_{1}\left(1+\beta_{2} \beta_{4}\right) \xi_{A}-\beta_{1}\left(\beta_{2}+\beta_{3}\right) \xi_{B}\right) \\
& \times \frac{1}{(1 \cdots k) \cdots(n+2 \cdots k-1)} \delta^{k \times 2}\left(C^{\prime} \cdot \underline{\underline{\lambda}}\right) \delta^{k \times 4}\left(C^{\prime} \cdot \underline{\underline{\eta}}\right) \delta^{(n+2-k) \times 2}\left(C^{\prime \perp} \cdot \underline{\lambda}\right) .
\end{aligned}
$$

Here, we introduced the following notation:

$$
\begin{array}{ll}
C_{n+1}^{\prime}=\frac{1}{1-\beta_{3} \beta_{4}} C_{n+1}+\frac{\beta_{3}}{1-\beta_{3} \beta_{4}} C_{n+2}, & C_{n+1}^{\perp}=C_{n+1}^{\perp}-\beta_{4} C_{n+2}^{\perp}, \\
C_{n+2}^{\prime}=\frac{1}{1-\beta_{3} \beta_{4}} C_{n+2}+\frac{\beta_{4}}{1-\beta_{3} \beta_{4}} C_{n+1}, & C_{n+2}^{\prime \perp}=C_{n+2}^{\perp}-\beta_{3} C_{n+1}^{\perp},
\end{array}
$$

and

$$
\begin{array}{lll}
\underline{\lambda}_{i}=\lambda_{i}, & i=1, \ldots, n, & \underline{\underline{\lambda}}_{n+1}=\xi_{A}, \quad \underline{\lambda}_{n+2}=\xi_{B}, \\
\stackrel{\tilde{\lambda}}{\underline{\lambda}}_{i}=\tilde{\lambda}_{i}, & i=1, \ldots, n, & \underline{\tilde{\lambda}}_{n+1}=\frac{\left\langle\xi_{B}\right| k}{\left\langle\xi_{B} \xi_{A}\right\rangle}, \quad \underline{\tilde{\lambda}}_{n+2}=-\frac{\left\langle\xi_{A}\right| k}{\left\langle\xi_{B} \xi_{A}\right\rangle} \\
\stackrel{\tilde{\eta}}{=}_{i}=\tilde{\eta}_{i}, & i=1, \ldots, n, & \stackrel{\tilde{\tilde{\eta}}}{=}_{n+1}=\tilde{\eta}_{p}, \quad \underline{\tilde{\eta}}_{n+2}=0 .
\end{array}
$$


The factor of $1 /\left(1-\beta_{3} \beta_{4}\right)^{2}$ in (A8) is the Jacobian from reorganizing the $C^{\perp} \cdot \lambda$ delta functions (see [74] for further details). Next, rewriting the first delta function in (A8) as

$$
\begin{aligned}
& \delta^{2}\left(\lambda_{p}+\beta_{1}\left(1+\beta_{2} \beta_{4}\right) \xi_{A}-\beta_{1}\left(\beta_{2}+\beta_{3}\right) \xi_{B}\right) \\
& \quad=\frac{1}{\beta_{1}^{2} \beta_{2}\left\langle\xi_{A} \xi_{B}\right\rangle} \delta\left(\beta_{3}-\frac{\left\langle\xi_{A} p\right\rangle}{\beta_{1}\left\langle\xi_{A} \xi_{B}\right\rangle}+\beta_{2}\right) \cdot \delta\left(\beta_{4}-\frac{\left\langle\xi_{B} p\right\rangle}{\beta_{1} \beta_{2}\left\langle\xi_{A} \xi_{B}\right\rangle}+\frac{1}{\beta_{2}}\right),
\end{aligned}
$$

choosing $\xi_{A}=\lambda_{p}, \xi_{B}=\xi$, and taking integrations over $\beta_{3}, \beta_{4}$, we get

$$
A_{k, n+1}^{*}=\frac{1}{\kappa^{*}}\langle\xi p\rangle \int \frac{d^{k \times(n+2)} C}{\operatorname{Vol}[G L(k)]} \frac{d \beta_{1} d \beta_{2}}{\beta_{1} \beta_{2}^{2}} \frac{\delta^{k \times 2}\left(C^{\prime} \cdot \underline{\underline{\lambda}}\right) \delta^{k \times 4}\left(C^{\prime} \cdot \underline{\underline{\tilde{\eta}}}\right) \delta^{(n+2-k) \times 2}\left(C^{\prime \perp} \cdot \underline{\underline{\lambda}}\right)}{(1 \cdots k)(2 \cdots k+1) \cdots(n+2 \cdots k-1)},
$$

where now

$$
\begin{array}{ll}
C_{n+1}^{\prime}=-\beta_{1} C_{n+1}+\beta_{1} \beta_{2} C_{n+2}, & C_{n+1}^{\prime \perp}=C_{n+1}^{\perp}+\frac{1+\beta_{1}}{\beta_{1} \beta_{2}} C_{n+2}^{\perp}, \\
C_{n+2}^{\prime}=-\beta_{1} C_{n+2}+\frac{1+\beta_{1}}{\beta_{2}} C_{n+1}, & C^{\prime \perp}{ }_{n+2}^{\perp}=C_{n+2}^{\perp}+\beta_{2} C_{n+1}^{\perp},
\end{array}
$$

and

$$
\begin{aligned}
& \underline{\underline{\lambda}}_{i}=\lambda_{i}, \quad i=1, \ldots, n, \quad \underline{\lambda}_{n+1}=\lambda_{p}, \quad \underline{\lambda}_{n+2}=\xi, \\
& \underline{\tilde{\lambda}}_{i}=\tilde{\lambda}_{i}, \quad i=1, \ldots, n, \quad \tilde{\tilde{\lambda}}_{n+1}=\frac{\langle\xi| k}{\langle\xi p\rangle}, \quad \tilde{\tilde{\lambda}}_{n+2}=-\frac{\langle p| k}{\langle\xi p\rangle}, \\
& \underline{\underline{\tilde{n}}}_{i}=\tilde{\eta}_{i}, \quad i=1, \ldots, n, \quad \underline{\underline{\tilde{\eta}}}_{n+1}=\tilde{\eta}_{p}, \quad \underline{\underline{\eta}}_{n+2}=0 .
\end{aligned}
$$

Introducing the inverse $C$-matrix transformation

$$
\begin{aligned}
& C_{n+1}=C_{n+1}^{\prime}+\beta_{2} C_{n+2}^{\prime}, \\
& C_{n+2}=\frac{1+\beta_{1}}{\beta_{1} \beta_{2}} C_{n+1}^{\prime}+C_{n+2}^{\prime}
\end{aligned}
$$

minors of the $C$ matrix containing both $n+1$ and $n+2$ columns when rewritten in terms of minors of the $C^{\prime}$ matrix acquire an extra $-\frac{1}{\beta_{1}}$ factor. For example, for $(n+1 \cdots k-2)$ minor we have

$$
(n+1 \cdots k-2)=-\frac{1}{\beta_{1}}(n+1 \cdots k-2)^{\prime} .
$$

This will generate a total power of $\left(\beta_{1}\right)^{k-1}$ in the numerator. Minors containing either $n+1$ or $n+2$ column transform as

$$
\begin{gathered}
(n+21 \cdots k-1)=\frac{1+\beta_{1}}{\beta_{1} \beta_{2}}(n+11 \cdots k-1)^{\prime}+(n+21 \cdots k-1)^{\prime}, \\
(n-k+2 \cdots n+1)=(n-k+2 \cdots n+1)^{\prime}+\beta_{2}(n-k+2 \cdots n n+2)^{\prime},
\end{gathered}
$$

while all other minors remain unchanged $(\cdots)=(\cdots)^{\prime}$. Finally, accounting for the Jacobian of transformation $\left(-\frac{1}{\beta_{1}}\right)^{k}$ we get

$$
\begin{aligned}
A_{k, n+1}^{*}= & -\frac{\langle\xi p\rangle}{\kappa^{*}} \int \frac{d^{k \times(n+2)} C^{\prime}}{\operatorname{Vol}[G L(k)]} \frac{d \beta_{1} d \beta_{2}}{\beta_{1} \beta_{2}} \delta^{k \times 2}\left(C^{\prime} \cdot \underline{\underline{\lambda}}\right) \delta^{k \times 4}\left(C^{\prime} \cdot \underline{\underline{\tilde{\eta}}}\right) \delta^{(n+2-k) \times 2}\left(C^{\prime \perp} \cdot \underline{\underline{\lambda}}\right) \\
& \times \frac{1}{(1 \cdots k)^{\prime} \cdots(n+2 \cdots k-1)^{\prime}\left(1+\beta_{2} \frac{(n-k+2 \cdots n n+2)^{\prime}}{(n-k+2 \cdots n n+1)^{\prime}}\right)\left(\beta_{1} \beta_{2}+\left(1+\beta_{1}\right) \frac{(n+11 \cdots k-1)}{(n+21 \cdots k-1)}\right)} .
\end{aligned}
$$


Now, understanding the integral over $\beta_{1,2}$ as a residue form and taking $\operatorname{res}_{\beta_{1}=-1}$ ores $_{\beta_{2}=0}$, we recover our result from [63]

$$
A_{k, n+1}^{*}=\int_{\Gamma_{k, n+2}^{\text {tree }}} \frac{d^{k \times(n+2)} C^{\prime}}{\operatorname{Vol}[G L(k)]} \operatorname{Reg} \frac{\delta^{k \times 2}\left(C^{\prime} \cdot \underline{\underline{\lambda}}\right) \delta^{k \times 4}\left(C^{\prime} \cdot \underline{\underline{\eta}}\right) \delta^{(n+2-k) \times 2}\left(C^{\prime \perp} \cdot \underline{\underline{\lambda}}\right)}{(1 \cdots k)^{\prime} \cdots(n+1 \cdots k-2)^{\prime}(n+21 \cdots k-1)^{\prime}},
$$

with

$$
\operatorname{Reg}=\frac{\langle\xi p\rangle}{\kappa^{*}} \frac{(n+21 \cdots k-1)^{\prime}}{(n+11 \cdots k-1)^{\prime}} .
$$

Now let us consider form factors of stress tensor supermultiplet operators. Let us consider (4.48). In this case we can write it as

$$
F_{k, n}=\left.\int \prod_{i=n+1}^{n+2} \frac{d^{2} \lambda_{i} d^{2} \tilde{\lambda}_{i}}{\operatorname{Vol}[G L(1)]} d^{4} \tilde{\eta}_{i} F_{2,2}\left(\Omega_{n+1}, \Omega_{n+2} ; \mathcal{T}\right)\right|_{\lambda \rightarrow-\lambda} A_{k, n+2}+\text { other gluing positions }
$$

where the minimal form factor $F_{2,2}\left(\Omega_{n+1}, \Omega_{n+2} ; \mathcal{T}\right)$ is given by (4.39). Performing next on-shell integrations for particles $n+1$ and $n+2$ as above ${ }^{31}$ we get (here we are considering only a single term, corresponding to the gluing of minimal form factor between legs 1 and $n$; other terms come from gluing between legs $i$ and $i+1, i=1 \cdots n-1$ )

$$
F_{k, n}=-\left\langle\xi_{A} \xi_{B}\right\rangle^{2} \int \frac{\mathrm{d} \beta_{1} \mathrm{~d} \beta_{2}}{\left(1-\beta_{1} \beta_{2}\right)^{2}} \int \frac{d^{k \times(n+2)} C}{\operatorname{Vol}[\operatorname{GL}(k)]} \frac{\delta^{k \times 2}\left(C^{\prime} \cdot \underline{\underline{\lambda}}\right) \delta^{k \times 4}\left(C^{\prime} \cdot \underline{\underline{\tilde{\eta}}}\right) \delta^{(n+2-k) \times 2}\left(C^{\prime \perp} \cdot \underline{\underline{\lambda}}\right)}{(1 \cdots k)(2 \cdots k+1) \cdots(n+2 \cdots k-1)},
$$

where

$$
\begin{array}{ll}
C_{n+1}^{\prime}=\frac{1}{1-\beta_{1} \beta_{2}} C_{n+1}+\frac{\beta_{1}}{1-\beta_{1} \beta_{2}} C_{n+2}, & C_{n+1}^{\perp}=C_{n+1}^{\perp}-\beta_{2} C_{n+2}^{\perp}, \\
C_{n+2}^{\prime}=\frac{1}{1-\beta_{1} \beta_{2}} C_{n+2}+\frac{\beta_{2}}{1-\beta_{1} \beta_{2}} C_{n+1}, & C_{n+2}^{\prime \perp}=C_{n+2}^{\perp}-\beta_{1} C_{n+1}^{\perp},
\end{array}
$$

and

$$
\begin{array}{lll}
\underline{\lambda}_{i}=\lambda_{i}, & i=1, \ldots, n, \quad \underline{\lambda}_{n+1}=\xi_{A}, \quad \underline{\underline{\lambda}}_{n+2}=\xi_{B}, \\
\tilde{\tilde{\lambda}}_{i}=\tilde{\lambda}_{i}, & i=1, \ldots, n, \quad \underline{\tilde{\lambda}}_{n+1}=-\frac{\left\langle\xi_{B}\right| q}{\left\langle\xi_{B} \xi_{A}\right\rangle}, \quad \underline{\tilde{\lambda}}_{n+2}=-\frac{\left\langle\xi_{A}\right| q}{\left\langle\xi_{A} \xi_{B}\right\rangle}, \\
\underline{\tilde{\eta}}_{i}^{+}=\tilde{\eta}_{i}^{+}, & i=1, \ldots, n, \quad & \underline{\tilde{\eta}}_{n+1}^{+}=0, \quad \tilde{\underline{\eta}}_{n+2}^{+}=0, \\
\underline{\tilde{\eta}}_{i}^{-}=\tilde{\eta}_{i}^{-}, & i=1, \ldots, n, \quad & \underline{\tilde{\eta}}_{n+1}^{-}=-\frac{\left\langle\xi_{B}\right| \gamma^{-}}{\left\langle\xi_{B} \xi_{A}\right\rangle}, \quad \underline{\tilde{\eta}}_{n+2}^{-}=-\frac{\left\langle\xi_{A}\right| \gamma^{-}}{\left\langle\xi_{A} \xi_{B}\right\rangle} .
\end{array}
$$

The transition from the integration over the $C$ matrix to the integration over the $C^{\prime}$ matrix is again done similar to the case of the off-shell amplitude considered above. This way our form factor is written as

$$
\begin{aligned}
F_{k, n}= & -\left\langle\xi_{A} \xi_{B}\right\rangle^{2} \int \frac{d^{k \times(n+2)} C^{\prime}}{\operatorname{Vol}[\operatorname{GL}(k)]} \frac{d \beta_{1} d \beta_{2}}{\left(1-\beta_{1} \beta_{2}\right)} \delta^{k \times 2}\left(C^{\prime} \cdot \underline{\underline{\lambda}}\right) \delta^{k \times 4}\left(C^{\prime} \cdot \underline{\underline{\eta}}\right) \delta^{(n+2-k) \times 2}\left(C^{\perp} \cdot \underline{\underline{\lambda}}\right) \\
& \times \frac{1}{(1 \cdots k)^{\prime} \cdots(n+2 \cdots k-1)^{\prime}\left(1-\beta_{1} \frac{(n-k+2 \cdots n n+2)^{\prime}}{(n-k+2 \cdots n n+1)^{\prime}}\right)\left(1-\beta_{2} \frac{(n+11 \cdots k-1)}{(n+21 \cdots k-1)}\right)}
\end{aligned}
$$

\footnotetext{
${ }^{31}$ See [74] for details.
} 
Finally, taking residues at $\beta_{1}=\frac{(n-k+2 \cdots n n+1)^{\prime}}{(n-k+2 \cdots n n+2)^{\prime}}$ and $\beta_{2}=\frac{(n+21 \cdots k-1)^{\prime}}{(n+11 \cdots k-1)^{\prime}}$ we reproduce the result of [74]

$$
F_{k, n}=\int \frac{d^{k \times(n+2)} C}{\operatorname{Vol}[\mathrm{GL}(k)]} \operatorname{Reg} \frac{\delta^{k \times 2}(C \cdot \underline{\underline{\lambda}}) \delta^{k \times 4}(C \cdot \underline{\underline{\tilde{\eta}}}) \delta^{(n+2-k) \times 2}\left(C^{\perp} \cdot \underline{\underline{\lambda}}\right)}{(1 \cdots k)(2 \cdots k+1) \cdots(n+2 \cdots k-1)},
$$

where now

$$
\operatorname{Reg}=\left\langle\xi_{A} \xi_{B}\right\rangle^{2} \frac{Y}{1-Y}, \quad Y=\frac{(n-k+2 \cdots n n+1)(n+21 \cdots k-1)}{(n-k+2 \cdots n n+2)(n+11 \cdots k-1)} .
$$

In the formula above we assumed a sum over different top-cell forms corresponding to different gluing positions of the minimal form factor. Note that the string correlation function knows about these different top cells by construction.

\section{APPENDIX B: GLUING OPERATOR FOR THE TREE AMPLITUDES}

In this appendix we present computational details for the application of gluing operator $\hat{A}_{i, i+1}$ to the tree level on-shell amplitudes. First of all, let us once more define $\hat{A}_{i, i+1}$ :

$$
\hat{A}_{i, i+1}[f] \equiv \int \prod_{i=n+1}^{n+2} \frac{d^{2} \lambda_{i} d^{2} \tilde{\lambda}_{i} d^{4} \eta}{\operatorname{Vol}[\mathrm{GL}(2)]} A_{2,2+1}^{*} \times f\left(\left\{\lambda_{i}, \tilde{\lambda}_{i}, \eta_{i}\right\}_{i=1}^{n+2}\right)
$$

Performing integration over $\tilde{\lambda}_{n+1}, \tilde{\lambda}_{n+2}, \tilde{\eta}_{n+1}$, and $\tilde{\eta}_{n+2}$ variables as in Appendix A, we get

$$
\hat{A}[f]=\left.\frac{\langle p \xi\rangle}{\kappa^{*}} \int \frac{d \beta_{1}}{\beta_{1}} \wedge \frac{d \beta_{2}}{\beta_{2}} \frac{1}{\beta_{1}^{2} \beta_{2}} f\left(\left\{\lambda_{i}, \tilde{\lambda}_{i}, \tilde{\eta}_{i}\right\}_{i=1}^{n+2}\right)\right|_{*},
$$

where $\left.\right|_{*}$ denotes substitutions $\left\{\lambda_{i}, \tilde{\lambda}_{i}, \eta_{i}\right\}_{i=n+1}^{n+2} \mapsto\left\{\lambda_{i}(\beta), \tilde{\lambda}_{i}(\beta), \tilde{\eta}_{i}(\beta)\right\}_{i=n+1}^{n+2}$ with

$$
\begin{aligned}
& \lambda_{n+1}(\beta)=\underline{\underline{\lambda}}_{n+1}+\beta_{2} \underline{\underline{\lambda}}_{n+2}, \quad \tilde{\lambda}_{n+1}(\beta)=\beta_{1} \stackrel{\tilde{\lambda}}{=}_{n+1}+\frac{\left(1+\beta_{1}\right)}{\beta_{2}} \underline{\tilde{\lambda}}_{n+2}, \quad \tilde{\eta}_{n+1}(\beta)=-\beta_{1} \tilde{\tilde{\eta}}_{n+1}, \\
& \lambda_{n+2}(\beta)=\underline{\underline{\lambda}}_{n+2}+\frac{\left(1+\beta_{1}\right)}{\beta_{1} \beta_{2}} \underline{\lambda}_{n+1}, \quad \tilde{\lambda}_{n+2}(\beta)=-\beta_{1} \underline{\underline{\lambda}}_{n+2}-\beta_{1} \beta_{2}{\underset{\tilde{\lambda}}{\underline{\lambda}}}_{n+1}, \quad \tilde{\eta}_{n+2}(\beta)=\beta_{1} \beta_{2} \underline{\underline{\eta}}_{n+1},
\end{aligned}
$$

and

$$
\underline{\underline{\lambda}}_{n+1}=\lambda_{p}, \quad \underline{\tilde{\lambda}}_{n+1}=\frac{\langle\xi| k}{\langle\xi p\rangle}, \quad \underline{\tilde{\eta}}_{n}=\tilde{\eta}_{p} ; \quad \underline{\underline{\lambda}}_{n+2}=\lambda_{\xi}, \quad \underline{\tilde{\lambda}}_{n+2}=\frac{\langle p| k}{\langle\xi p\rangle}, \quad \underline{\tilde{\eta}}_{n+2}=0
$$

We understand integration with respect to $\beta_{1,2}$ as a residue form, and we will always evaluate it at points $r e s_{\beta_{2}=0} \circ \operatorname{res}_{\beta_{1}=-1}$.

The following formulas are useful in computations. The transformed momenta for $n+$ first and $n+$ second particles are then given by

$$
\begin{aligned}
& p_{n+1}(\beta)=-\beta_{1} \underline{\underline{\lambda}}_{n+1} \underline{\tilde{\lambda}}_{n+1}+\frac{1+\beta_{1}}{\beta_{2}} \underline{\underline{\lambda}}_{n+1} \stackrel{\tilde{\lambda}}{n}_{n+2}-\beta_{1} \beta_{2} \underline{\underline{\lambda}}_{n+2} \stackrel{\tilde{\lambda}}{=}_{n+1}+\left(1+\beta_{1}\right) \underline{\underline{\lambda}}_{n+2} \underline{\tilde{\lambda}}_{n+2}, \\
& p_{n+2}(\beta)=-\beta_{1} \underline{\underline{\lambda}}_{n+2} \underline{\tilde{\lambda}}_{n+2}-\frac{1+\beta_{1}}{\beta_{2}} \underline{\underline{\lambda}}_{n+1} \underline{\tilde{\lambda}}_{n+2}+\beta_{1} \beta_{2} \underline{\underline{\lambda}}_{n+2} \stackrel{\tilde{\lambda}}{=}_{n+1}+\left(1+\beta_{1}\right) \underline{\underline{\lambda}}_{n+1} \underline{\tilde{\lambda}}_{n+1},
\end{aligned}
$$

and using definitions above it is easy to see that

$$
\begin{aligned}
k & =\lambda_{n+1}(\beta) \tilde{\lambda}_{n+1}(\beta)+\lambda_{n+2}(\beta) \tilde{\lambda}_{n+2}(\beta), \\
\lambda_{p} \eta_{p} & =\lambda_{n+1}(\beta) \tilde{\eta}_{n+1}(\beta)+\lambda_{n+2}(\beta) \tilde{\eta}_{n+2}(\beta),
\end{aligned}
$$

for all values of $\beta_{1}$ and $\beta_{2}$. 
First of all, let us make a comment for example (6.9) considered in the main text that the gluing operation commutes with projectors on particular physical particles provided we identify $n+1$ and $n+2$ particles with gluons with -+ polarizations. Indeed from the previous example we have $[53,63]\left[A_{2,4} \equiv A_{2,4}\left(\Omega_{1}, \ldots, \Omega_{4}\right)\right]$

$$
\partial_{\tilde{\eta}_{2}}^{4} \partial_{\tilde{\eta}_{p}}^{4} \hat{A}\left[A_{2,4}\right]=\hat{A}\left[\partial_{\tilde{\eta}_{2}}^{4} \partial_{\tilde{\eta}_{3}}^{4} A_{2,4}\right]=\frac{\delta^{4}\left(p_{12}+k\right)}{\kappa^{*}\langle 12\rangle} \operatorname{res}_{\beta_{1}=-1} \operatorname{ores}_{\beta_{2}=0}[\omega]=A_{2,2+1}^{*}\left(1^{+} 2^{-} g_{3}^{*}\right),
$$

where $\omega$ is now given by

$$
\omega=\frac{\left(\langle 2 p\rangle+\beta_{2}\langle 2 \xi\rangle\right)^{3} d \beta_{2} \wedge d \beta_{1}}{\beta_{2} \beta_{1}\left(\beta_{1} \beta_{2}\langle 1 \xi\rangle+\left(1+\beta_{1}\right)\langle 1 p\rangle\right)} .
$$

Now let us proceed with more involved examples considered in the text and reproduce results for $A_{3,3+1}^{*}\left(1^{-} 2^{-} 3^{+} g_{4}^{*}\right)$ and $A_{3,4+1}^{*}\left(1^{+} 2^{+} 3^{-} 4^{-} g_{5}^{*}\right)$ amplitudes from [63]. In the case of the $A_{3,3+1}^{*}\left(1^{-} 2^{-} 3^{+} g_{4}^{*}\right)$ amplitude we have to start with the $A_{3,5}\left(1^{-} 2^{-} 3^{+} 4^{-} 5^{+}\right)$amplitude (here and below $\left.c^{-1}=\langle p \xi\rangle\right)$,

$$
\partial_{\tilde{\eta}_{1}}^{4} \partial_{\tilde{\eta}_{2}}^{4} \partial_{\tilde{\eta}_{4}}^{4} A_{3,5}\left(\Omega_{1}, \ldots, \Omega_{5}\right)=A_{3,5}\left(1^{-} 2^{-} 3^{+} 4^{-} 5^{+}\right)=\delta^{4}\left(p_{12345}\right) \frac{[35]^{4}}{[12][23][34][45][51]},
$$

so that

$$
\left.A_{3,5}\left(1^{-} 2^{-} 3^{+} 4^{-} 5^{+}\right)\right|_{*}=\frac{\delta^{4}\left(p_{123}+k\right) \beta_{1}^{2} \beta_{2}\left(\kappa^{*} c^{-1}[p 3]+\beta_{2}[3 \underline{\underline{4}}]\right)^{4}}{k^{2} c^{-1}[12][23]\left(-\beta_{1} \beta_{2}[3 \underline{\underline{4}}]+\left(1+\beta_{1}\right) c^{-1} \kappa^{*}[3 p]\right)\left([1 p] c^{-1} \kappa^{*}+\beta_{2}[1 \underline{\underline{4}}]\right)} .
$$

Now, recalling that $k^{2}=-\kappa^{*} \kappa$, we get [63]

$$
\hat{A}_{45}\left[A_{3,5}\left(1^{-} 2^{-} 3^{+} 4^{-} 5^{+}\right)\right]=\frac{\delta^{4}\left(p_{123}+k\right)[p 3]^{3}}{\kappa[12][23][p 1]}=A_{2,3+1}^{*}\left(1^{-} 2^{-} 3^{+} g_{4}^{*}\right)
$$

and the integration with respect to $\beta$ 's was performed by taking composite residue $\operatorname{res}_{\beta_{1}=-1} \circ{ }^{\circ e s} s_{\beta_{2}=0}[\cdots]$.

In a similar fashion for the $A_{3,5}\left(1^{+} 2^{+} 3^{-} 4^{-} 5^{-} 6^{+}\right)$amplitude we have

$$
\partial_{\tilde{\eta}_{3}}^{4} \partial_{\tilde{\eta}_{4}}^{4} \partial_{\tilde{\eta}_{5}}^{4} A_{3,5}\left(\Omega_{1}, \ldots, \Omega_{6}\right)=A_{3,5}\left(1^{+} 2^{+} 3^{-} 4^{-} 5^{-} 6^{+}\right)=A+B
$$

with

$$
\begin{aligned}
& A=\frac{\langle 3|1+2| 6]^{3}}{[45][56]} \frac{\delta^{4}\left(p_{1 \ldots 6}\right)}{\langle 12\rangle\langle 23\rangle p_{1,3}^{2}\langle 1|2+3| 4]}, \\
& B=\frac{\langle 5|3+4| 2]^{3}}{\langle 56\rangle\langle 61\rangle} \frac{\delta^{4}\left(p_{1 \ldots 6}\right)}{[23][34] p_{2,4}^{2}\langle 1|2+3| 4]} .
\end{aligned}
$$

Next, it is not hard to see that $[[x|\equiv\langle 3|(1+2)| y,\rangle \equiv(3+4)| 2]]$

$$
\begin{aligned}
\left.A\right|_{*} & =\frac{\beta_{1}^{2} \beta_{2}\left([p x] c^{-1} \kappa^{*}+\beta_{2}[\underline{\underline{5}} x]\right)^{3}}{c^{-1} \kappa \kappa^{*}\left(\beta_{1}^{2} \beta_{2}[4 \underline{\underline{5}}]+\left(1+\beta_{1}\right)[4 p] \kappa^{*} c^{-1}\right)} \frac{\delta^{4}\left(p_{1234}+k\right)}{\langle 12\rangle\langle 23\rangle p_{1,3}^{2}\langle 1|2+3| 4]}, \\
\left.B\right|_{*} & =\frac{\beta_{1}^{2} \beta_{2}\left(\langle p y\rangle+\beta_{2}\langle\xi y\rangle\right)^{3}}{c\left(\langle 1 \xi\rangle \beta_{1} \beta_{2}+\left(1+\beta_{1}\right)\langle p 1\rangle\right)} \frac{\delta^{4}\left(p_{1234}+k\right)}{[23][34] p_{2,4}^{2}\langle 1|2+3| 4]} .
\end{aligned}
$$


Now, defining

$$
\begin{aligned}
& \omega_{A}=\frac{\left([p x] c^{-1} \kappa^{*}+\beta_{2}[\underline{\underline{5}} x]\right)^{3}}{c^{-1} \kappa \kappa^{*}\left(\beta_{1} \beta_{2}[4 \underline{\underline{5}}]+\left(1+\beta_{1}\right)[4 p] \kappa^{*} c^{-1}\right)} \frac{d \beta_{2} \wedge d \beta_{1}}{\beta_{1} \beta_{2}}, \\
& \omega_{B}=\frac{\left(\langle p y\rangle+\beta_{2}\langle\xi y\rangle\right)^{3}}{c\left(\langle 1 \xi\rangle \beta_{1} \beta_{2}+\left(1+\beta_{1}\right)\langle p 1\rangle\right)} \frac{d \beta_{2} \wedge d \beta_{1}}{\beta_{1} \beta_{2}},
\end{aligned}
$$

we get

$$
\begin{aligned}
\hat{A}_{56}[A] & =\frac{\delta^{4}\left(p_{1234}+k\right)}{\langle 12\rangle\langle 23\rangle p_{1,3}^{2}\langle 1|2+3| 4]} \frac{1}{c^{-1} \kappa^{*}} \operatorname{res}_{\beta_{1}=-1} \operatorname{ores}_{\beta_{2}=0}\left[\omega_{A}\right] \\
& =\frac{1}{\kappa} \frac{\delta^{4}\left(p_{1234}+k\right)\langle 3|1+2| p]^{3}}{\langle 12\rangle\langle 23\rangle[4 p] p_{1,3}^{2}\langle 1|2+3| 4]}
\end{aligned}
$$

and

$$
\begin{aligned}
\hat{A}_{56}[B] & =\frac{\delta^{4}\left(p_{1234}+k\right)}{[23][34] p_{2,4}^{2}\langle 1|2+3| 4]} \frac{1}{c^{-1} \kappa^{*}} \operatorname{res}_{\beta_{1}=-1} \operatorname{ores}_{\beta_{2}=0}\left[\omega_{B}\right] \\
& =\frac{1}{\kappa^{*}} \frac{\delta^{4}\left(p_{1234}+k\right)\langle p|3+4| 2]^{3}}{\langle p 1\rangle[23][34] p_{2,4}^{2}\langle 1|2+3| 4]}
\end{aligned}
$$

So, as expected [63],

$$
\hat{A}_{56}\left[A_{3,5}\left(1^{+} 2^{+} 3^{-} 4^{-} 5^{-} 6^{+}\right)\right]=A_{3,4+1}^{*}\left(1^{+} 2^{+} 3^{-} 4^{-} g_{5}^{*}\right) .
$$

As a final example, let us consider the following example. Let us reproduce the $A_{3,0+3}^{*}\left(g_{1}^{*}, g_{2}^{*}, g_{3}^{*}\right)$ Wilson line correlation function from the $A_{3,6}\left(1^{-} 2^{+} 3^{-} 4^{+} 5^{-} 6^{+}\right)$onshell amplitude. According to our previous discussion, $A_{3,0+3}^{*}$ could be written as

$$
\left.A_{3,0+3}^{*}\left(g_{1}^{*}, g_{2}^{*}, g_{3}^{*}\right)=\left(\hat{A}_{12} \circ \hat{A}_{34} \circ \hat{A}_{56}\right)\left[A_{3,6}\left(1^{-} 2^{+} 3^{-} 4^{+} 5^{-} 6^{+}\right)\right]\right] \text {, }
$$

where the $A_{3,6}\left(1^{-} 2^{+} 3^{-} 4^{+} 5^{-} 6^{+}\right)$amplitude is given by

$$
\begin{aligned}
A_{3,6} & =\delta^{4}\left(p_{1 \cdots 6}\right)\left(1+\mathbb{P}^{2}+\mathbb{P}^{4}\right) f, f \\
& =\frac{\langle 13\rangle^{4}[46]^{4}}{\langle 12\rangle\langle 23\rangle[45][56]\langle 3|1+2| 6]\langle 1|5+6| 4] p_{456}^{2}}
\end{aligned}
$$

and $\mathbb{P}$ is the permutation operator shifting spinor labels by +1 mod 6 . The algebraic manipulation related to the actions of $\hat{A}_{i i+1}$ operators are identical to those already discussed. The factors $1 / \beta_{1}^{2} \beta_{2}$ in the definition of gluing operators will cancel with corresponding factors in the amplitude after substitutions are applied, while integrals are evaluated by composite residues res $\mathrm{r}_{\beta_{1}=-1} \circ \mathrm{res}_{\beta_{2}=0}$. So, in what follows we will present only the results of applying gluing operators $\hat{A}_{i i+1}$ to the on-shell amplitude. For the $f$ term we have

$$
\begin{aligned}
\hat{A}_{56}[f]= & \delta^{4}\left(p_{1234}+k_{3}\right) \\
& \times \frac{\langle 13\rangle^{4}\left[4 p_{3}\right]^{3}}{\kappa_{3}\langle 12\rangle\langle 23\rangle\left\langle 3|1+2| p_{3}\right]\left\langle 1\left|k_{3}\right| 4\right] p_{123}^{2}}
\end{aligned}
$$

and

$$
\begin{aligned}
& \left(\hat{A}_{34} \circ \hat{A}_{56}\right)[f] \\
& =\delta^{4}\left(p_{12}+k_{2}+k_{3}\right) \\
& \quad \times \frac{\left\langle 1 p_{2}\right\rangle^{4}\left[p_{2} p_{3}\right]^{3}}{\kappa_{3}\langle 12\rangle\left\langle 2 p_{2}\right\rangle\left\langle p_{2}|1+2| p_{3}\right]\left\langle 1\left|k_{3}\right| p_{2}\right]\left\langle p_{2}\left|k_{3}\right| p_{2}\right]} .
\end{aligned}
$$

Note that the ordinary propagator $1 / p_{123}^{2}$ transformed into eikonal one $1 /\left(p_{2} k_{3}\right)$ after the action of the gluing operator. Finally

$$
\begin{aligned}
\left(\hat{A}_{12} \circ \hat{A}_{34} \circ \hat{A}_{56}\right)[f]= & \delta^{4}\left(k_{1}+k_{2}+k_{3}\right) \\
& \times \frac{\left\langle p_{1} p_{2}\right\rangle^{3}\left[p_{2} p_{3}\right]^{3}}{\kappa_{3} \kappa_{1}^{*}\left\langle p_{2}\left|k_{1}\right| p_{3}\right]\left\langle p_{1}\left|k_{3}\right| p_{2}\right]\left\langle p_{2}\left|k_{1}\right| p_{2}\right]},
\end{aligned}
$$

where we used that $\left\langle p_{2}\left|k_{3}\right| p_{2}\right]=\left\langle p_{2}\left|k_{1}\right| p_{2}\right]$. Other terms can be obtained by similar computations or just by careful relabeling of indexes. The final result takes the form

$$
\begin{aligned}
A_{3,0+3}^{*} & =\left(\hat{A}_{12} \circ \hat{A}_{34} \circ \hat{A}_{56}\right)\left[A_{3,6}\left(1^{-} 2^{+} 3^{-} 4^{+} 5^{-} 6^{+}\right)\right] \\
& =\delta^{4}\left(k_{1}+k_{2}+k_{3}\right)\left(1+\mathbb{P}^{\prime}+\mathbb{P}^{\prime 2}\right) \tilde{f}, \\
\tilde{f} & =\frac{\left\langle p_{1} p_{2}\right\rangle^{3}\left[p_{2} p_{3}\right]^{3}}{\kappa_{3} \kappa_{1}^{*}\left\langle p_{2}\left|k_{1}\right| p_{3}\right]\left\langle p_{1}\left|k_{3}\right| p_{2}\right]\left\langle p_{2}\left|k_{1}\right| p_{2}\right]} .
\end{aligned}
$$

Here $\mathbb{P}^{\prime}$ is the permutation operator that now shifts all spinor and momenta labels by $+1 \bmod 3$. The expression obtained is in full agreement with previous computations using both Grassmannian integral representation [64] and $\mathrm{BCFW}$ recursion [53]. 
[1] E. Witten, Perturbative gauge theory as a string theory in twistor space, Commun. Math. Phys. 252, 189 (2004).

[2] N. Berkovits, An Alternative String Theory in Twistor Space for $N=4$ Super-Yang-Mills, Phys. Rev. Lett. 93, 011601 (2004).

[3] R. Roiban, M. Spradlin, and A. Volovich, On the tree level $S$ matrix of Yang-Mills theory, Phys. Rev. D 70, 026009 (2004).

[4] M. Spradlin and A. Volovich, From twistor string theory to recursion relations, Phys. Rev. D 80, 085022 (2009).

[5] F. Cachazo, S. He, and E. Y. Yuan, Scattering equations and Kawai-Lewellen-Tye orthogonality, Phys. Rev. D 90, 065001 (2014).

[6] F. Cachazo, S. He, and E. Y. Yuan, Scattering of Massless Particles in Arbitrary Dimensions, Phys. Rev. Lett. 113, 171601 (2014).

[7] F. Cachazo, S. He, and E. Y. Yuan, Scattering of massless particles: Scalars, gluons and gravitons, J. High Energy Phys. 07 (2014) 033.

[8] F. Cachazo, S. He, and E. Y. Yuan, Einstein-Yang-Mills scattering amplitudes from scattering equations, J. High Energy Phys. 01 (2015) 121.

[9] F. Cachazo, S. He, and E. Y. Yuan, Scattering equations and matrices: From Einstein to Yang-Mills, DBI and NLSM, J. High Energy Phys. 07 (2015) 149.

[10] L. Mason and D. Skinner, Ambitwistor strings and the scattering equations, J. High Energy Phys. 07 (2014) 048.

[11] Y. Geyer, A. E. Lipstein, and L. J. Mason, Ambitwistor Strings in Four Dimensions, Phys. Rev. Lett. 113, 081602 (2014).

[12] T. Adamo, E. Casali, and D. Skinner, Ambitwistor strings and the scattering equations at one loop, J. High Energy Phys. 04 (2014) 104.

[13] K. Ohmori, Worldsheet geometries of ambitwistor string, J. High Energy Phys. 06, (2015) 075.

[14] E. Casali and P. Tourkine, Infrared behavior of the oneloop scattering equations and supergravity integrands, J. High Energy Phys. 04 (2015) 013.

[15] T. Adamo and E. Casali, Scattering equations, supergravity integrands, and pure spinors, J. High Energy Phys. 05 (2015) 120.

[16] Y. Geyer, L. Mason, R. Monteiro, and P. Tourkine, Loop Integrands for Scattering Amplitudes from the Riemann Sphere, Phys. Rev. Lett. 115, 121603 (2015).

[17] Y. Geyer, L. Mason, R. Monteiro, and P. Tourkine, Oneloop amplitudes on the Riemann sphere, J. High Energy Phys. 03 (2016) 114.

[18] C. Cardona and H. Gomez, Elliptic scattering equations, J. High Energy Phys. 06 (2016) 094.

[19] C. Cardona and H. Gomez, CHY-graphs on a torus, J. High Energy Phys. 10 (2016) 116.

[20] F. Cachazo, S. He, and E. Y. Yuan, One-loop corrections from higher dimensional tree amplitudes, J. High Energy Phys. 08 (2016) 008.

[21] Y. Geyer, L. Mason, R. Monteiro, and P. Tourkine, Twoloop scattering amplitudes from the Riemann sphere, Phys. Rev. D 94, 125029 (2016).
[22] N. Arkani-Hamed, F. Cachazo, C. Cheung, and J. Kaplan, A duality for the $S$ matrix, J. High Energy Phys. 03 (2010) 020 .

[23] N. Arkani-Hamed, J. L. Bourjaily, F. Cachazo, A. B. Goncharov, A. Postnikov, and J. Trnka, Scattering Amplitudes and the Positive Grassmannian (Cambridge University Press, Cambridge, UK, 2012).

[24] N. Arkani-Hamed, J. L. Bourjaily, F. Cachazo, S. CaronHuot, and J. Trnka, The all-loop integrand for scattering amplitudes in planar $N=4$ SYM, J. High Energy Phys. 01 (2011) 041.

[25] N. Arkani-Hamed, F. Cachazo, and C. Cheung, The Grassmannian origin of dual superconformal invariance, J. High Energy Phys. 03 (2010) 036.

[26] N. Arkani-Hamed, J. Bourjaily, F. Cachazo, and J. Trnka, Unification of residues and Grassmannian dualities, J. High Energy Phys. 01 (2011) 049.

[27] L. J. Mason and D. Skinner, Dual superconformal invariance, momentum twistors and Grassmannians, J. High Energy Phys. 11 (2009) 045.

[28] R. Britto, F. Cachazo, and B. Feng, New recursion relations for tree amplitudes of gluons, Nucl. Phys. B715, 499 (2005).

[29] R. Britto, F. Cachazo, B. Feng, and E. Witten, Direct Proof of Tree-Level Recursion Relation in Yang-Mills Theory, Phys. Rev. Lett. 94, 181602 (2005).

[30] J. M. Drummond, J. M. Henn, and J. Plefka, Yangian symmetry of scattering amplitudes in $N=4$ super Yang-Mills theory, J. High Energy Phys. 05 (2009) 046.

[31] J. M. Drummond, J. Henn, G. P. Korchemsky, and E. Sokatchev, Dual superconformal symmetry of scattering amplitudes in $N=4$ super-Yang-Mills theory, Nucl. Phys. B828, 317 (2010).

[32] D. Chicherin, S. Derkachov, and R. Kirschner, YangBaxter operators and scattering amplitudes in $N=4$ super-Yang-Mills theory, Nucl. Phys. B881, 467 (2014).

[33] R. Frassek, N. Kanning, Y. Ko, and M. Staudacher, Bethe ansatz for Yangian invariants: Towards super Yang-Mills scattering amplitudes, Nucl. Phys. B883, 373 (2014).

[34] N. Beisert, A. Garus, and M. Rosso, Yangian Symmetry and Integrability of Planar $N=4$ Super-Yang-Mills Theory, Phys. Rev. Lett. 118, 141603 (2017).

[35] A. Hodges, Eliminating spurious poles from gaugetheoretic amplitudes, J. High Energy Phys. 05 (2013) 135.

[36] N. Arkani-Hamed, J. L. Bourjaily, F. Cachazo, A. Hodges, and J. Trnka, A note on polytopes for scattering amplitudes, J. High Energy Phys. 04 (2012) 081.

[37] N. Arkani-Hamed and J. Trnka, The amplituhedron, J. High Energy Phys. 10 (2014) 030.

[38] N. Arkani-Hamed and J. Trnka, Into the amplituhedron, J. High Energy Phys. 12 (2014) 182.

[39] Y. Bai and S. He, The amplituhedron from momentum twistor diagrams, J. High Energy Phys. 02 (2015) 065.

[40] S. Franco, D. Galloni, A. Mariotti, and J. Trnka, Anatomy of the amplituhedron, J. High Energy Phys. 03 (2015) 128.

[41] Z. Bern, E. Herrmann, S. Litsey, J. Stankowicz, and J. Trnka, Evidence for a nonplanar amplituhedron, J. High Energy Phys. 06 (2016) 098.

[42] L. Ferro, T. Lukowski, A. Orta, and M. Parisi, Towards the amplituhedron volume, J. High Energy Phys. 03 (2016) 014 . 
[43] H. Elvang and Y.-t. Huang, Scattering amplitudes, arXiv:1308.1697.

[44] L. V. Bork, On NMHV form factors in $N=4$ SYM theory from generalized unitarity, J. High Energy Phys. 01 (2013) 049.

[45] L. V. Bork, On form factors in $\mathcal{N}=4$ SYM theory and polytopes, J. High Energy Phys. 12 (2014) 111.

[46] A. Brandhuber, B. Spence, G. Travaglini, and G. Yang, Form factors in $N=4$ super Yang-Mills and periodic Wilson loops, J. High Energy Phys. 01 (2011) 134.

[47] G. Yang, Color-kinematics duality and Sudakov form factor at five loops for $N=4$ supersymmetric Yang-Mills theory, Phys. Rev. Lett. 117, 271602 (2016).

[48] J. Maldacena and A. Zhiboedov, Form factors at strong coupling via a Y-system, J. High Energy Phys. 11 (2010) 104.

[49] Z. Gao and G. Yang, Y-system for form factors at strong coupling in $\mathrm{AdS}_{5}$ and with multi-operator insertions in $\mathrm{AdS}_{3}$, J. High Energy Phys. 06 (2013) 105.

[50] M. Wilhelm, Amplitudes, form factors and the dilatation operator in $\mathcal{N}=4$ SYM theory, J. High Energy Phys. 02 (2015) 149.

[51] D. Nandan, C. Sieg, M. Wilhelm, and G. Yang, Cutting through form factors and cross sections of non-protected operators in $\mathcal{N}=4$ SYM, J. High Energy Phys. 06 (2015) 156.

[52] F. Loebbert, D. Nandan, C. Sieg, M. Wilhelm, and G. Yang, On-shell methods for the two-loop dilatation operator and finite remainders, J. High Energy Phys. 10 (2015) 012.

[53] A. van Hameren, BCFW recursion for off-shell gluons, J. High Energy Phys. 07 (2014) 138.

[54] A. van Hameren and M. Serino, BCFW recursion for TMD parton scattering, J. High Energy Phys. 07 (2015) 010.

[55] K. Kutak, A. Hameren, and M. Serino, QCD amplitudes with 2 initial spacelike legs via generalised BCFW recursion, J. High Energy Phys. 02 (2017) 009.

[56] L. N. Lipatov, Gauge invariant effective action for high-energy processes in QCD, Nucl. Phys. B452, 369 (1995).

[57] L. N. Lipatov, Small $x$ physics in perturbative QCD, Phys. Rep. 286, 131 (1997).

[58] R. Kirschner, L. N. Lipatov, and L. Szymanowski, Effective action for multi-Regge processes in QCD, Nucl. Phys. B425, 579 (1994).

[59] R. Kirschner, L. N. Lipatov, and L. Szymanowski, Symmetry properties of the effective action for high-energy scattering in QCD, Phys. Rev. D 51, 838 (1995).

[60] P. Kotko, Wilson lines and gauge invariant off-shell amplitudes, J. High Energy Phys. 07 (2014) 128.

[61] A. van Hameren, P. Kotko, and K. Kutak, Multi-gluon helicity amplitudes with one off-shell leg within high energy factorization, J. High Energy Phys. 12 (2012) 029.

[62] A. van Hameren, P. Kotko, and K. Kutak, Helicity amplitudes for high-energy scattering, J. High Energy Phys. 01 (2013) 078.

[63] L. V. Bork and A. I. Onishchenko, Wilson lines, Grassmannians and gauge invariant off-shell amplitudes in $N=$ 4 SYM, J. High Energy Phys. 04 (2017) 019.
[64] L. V. Bork and A. I. Onishchenko, Grassmannian integral for general gauge invariant off-shell amplitudes in $N=4$ SYM ,J. High Energy Phys. 05 (2017) 019.(2016).

[65] D. Chicherin, P. Heslop, G. P. Korchemsky, and E. Sokatchev, Wilson loop form factors: A new duality arXiv:1612.05197.

[66] L. V. Gribov, E. M. Levin, and M. G. Ryskin, Semihard processes in QCD, Phys. Rep. 100, 1 (1983).

[67] S. Catani, M. Ciafaloni, and F. Hautmann, High-energy factorization and small $x$ heavy flavor production, Nucl. Phys. B366, 135 (1991).

[68] J. C. Collins and R. K. Ellis, Heavy quark production in very high-energy hadron collisions, Nucl. Phys. B360, 3 (1991).

[69] S. Catani and F. Hautmann, High-energy factorization and small $x$ deep inelastic scattering beyond leading order, Nucl. Phys. B427, 475 (1994).

[70] A. Brandhuber, E. Hughes, R. Panerai, B. Spence, and G. Travaglini, The connected prescription for form factors in twistor space, J. High Energy Phys. 11 (2016) 143.

[71] S. He and Z. Liu, A note on connected formula for form factors, J. High Energy Phys. 12 (2016) 006.

[72] S. He and Y. Zhang, Connected formulas for amplitudes in standard model ,J. High Energy Phys. 03 (2017) 093..

[73] L. V. Bork and A. I. Onishchenko, On soft theorems and form factors in $\mathcal{N}=4 \mathrm{SYM}$ theory, J. High Energy Phys. 12 (2015) 030.

[74] R. Frassek, D. Meidinger, D. Nandan, and M. Wilhelm, On-shell diagrams, Gramannians and integrability for form factors, J. High Energy Phys. 01 (2016) 182.

[75] M. Wilhelm, Form factors and the dilatation operator in $\mathcal{N}=4$ super Yang-Mills theory and its deformations, Ph.D. thesis, Humboldt University, Berlin, 2016.

[76] L. V. Bork and A. I. Onishchenko, Grassmannians and form factors with $q^{2}=0$ in $\mathcal{N}=4$ SYM theory, J. High Energy Phys. 12 (2016) 076.

[77] L. Koster, V. Mitev, M. Staudacher, and M. Wilhelm, Composite Operators in the Twistor Formulation of $N=4$ Supersymmetric Yang-Mills Theory, Phys. Rev. Lett. 117, 011601 (2016).

[78] L. Koster, V. Mitev, M. Staudacher, and M. Wilhelm, All tree-level MHV form factors in $\mathcal{N}=4$ SYM from twistor space, J. High Energy Phys. 06 (2016) 162.

[79] L. Koster, V. Mitev, M. Staudacher, and M. Wilhelm, On form factors and correlation functions in twistor space, J. High Energy Phys. 03 (2017) 131.

[80] D. Chicherin and E. Sokatchev, $\mathcal{N}=4$ super-Yang-Mills in LHC superspace part I: Classical and quantum theory, J. High Energy Phys. 02 (2017) 062.

[81] D. Chicherin and E. Sokatchev, $N=4$ super-Yang-Mills in LHC superspace. Part II: Non-chiral correlation functions of the stress-tensor multiplet, J. High Energy Phys. 03 (2017) 048 .

[82] D. Chicherin and E. Sokatchev, Demystifying the twistor construction of composite operators in $N=4$ super-Yang-Mills theory, J. Phys. A Math. Theor. 50, 205402 (2016).

[83] D. Chicherin and E. Sokatchev, Composite operators and form factors in $N=4 \mathrm{SYM}$, J. Phys. A Math. Theor. 50, 275402 (2016). 
[84] L. V. Bork and A. I. Onishchenko, Ambitwistor strings and Reggeon amplitudes in $N=4$ SYM, Phys. Lett. B 774, 403 (2017).

[85] F. Gliozzi, J. Scherk, and D. I. Olive, Supersymmetry, supergravity theories and the dual spinor model, Nucl. Phys. B122, 253 (1977).

[86] L. Brink, J. H. Schwarz, and J. Scherk, Supersymmetric Yang-Mills theories, Nucl. Phys. B121, 77 (1977).

[87] V.P. Nair, A current algebra for some gauge theory amplitudes, Phys. Lett. B 214, 215 (1988).

[88] L. J. Dixon, Calculating scattering amplitudes efficiently, in $Q C D$ and Beyond. Proceedings of the Theoretical Advanced Study Institute in Elementary Particle Physics, TASI-95, Boulder, CO, 1995 (World Scientific, Singapore, 1996), pp. 539-584.

[89] G. G. Hartwell and P. S. Howe, $(N, p, q)$ harmonic superspace, Int. J. Mod. Phys. A 10, 3901 (1995).

[90] B. Eden, P. Heslop, G. P. Korchemsky, and E. Sokatchev, The super-correlator/super-amplitude duality: Part I, Nucl. Phys. B869, 329 (2013).

[91] A. Brandhuber, O. Gurdogan, R. Mooney, G. Travaglini, and G. Yang, Harmony of super form factors, J. High Energy Phys. 10 (2011) 046.

[92] L. V. Bork, D. I. Kazakov, and G. S. Vartanov, On MHV form factors in superspace for $N=4$ SYM theory, J. High Energy Phys. 10 (2011) 133.

[93] B. Penante, B. Spence, G. Travaglini, and C. Wen, On super form factors of half-BPS operators in $N=4$ super Yang-Mills, J. High Energy Phys. 04 (2014) 083.

[94] Y. Geyer, Ambitwistor strings: Worldsheet approaches to perturbative quantum field theories, Ph.D. thesis, Oxford University, 2016.

[95] M. Ademollo, A. Bellini, and M. Ciafaloni, Superstring Regge amplitudes and emission vertices, Phys. Lett. B 223, 318 (1989).

[96] M. Ademollo, A. Bellini, and M. Ciafaloni, Superstring Regge amplitudes and graviton radiation at Planckian energies, Nucl. Phys. B338, 114 (1990).

[97] N. Arkani-Hamed, F. Cachazo, and J. Kaplan, What is the simplest quantum field theory?, J. High Energy Phys. 09 (2010) 016.

[98] A. A. Rosly and K. G. Selivanov, On amplitudes in self-dual sector of Yang-Mills theory, Phys. Lett. B 399, 135 (1997).

[99] N. Arkani-Hamed, J. Bourjaily, F. Cachazo, and J. Trnka, Local spacetime physics from the Grassmannian, J. High Energy Phys. 01 (2011) 108.

[100] D. Meidinger, D. Nandan, B. Penante, and C. Wen, A note on NMHV form factors from the Gramannian and the twistor string, J. High Energy Phys. 09 (2017) 024.

[101] N. Beisert and A. Garus, Yangian algebra and correlation functions in planar gauge theories, arXiv:1804.09110.
[102] N. Beisert, A. Garus, and M. Rosso, Yangian symmetry for the action of planar $\mathcal{N}=4$ super Yang-Mills and $\mathcal{N}=6$ super Chern-Simons theories, arXiv:1803.06310.

[103] L. Dolan and P. Goddard, Gluon tree amplitudes in open twistor string theory, J. High Energy Phys. 12 (2009) 032.

[104] D. Nandan, A. Volovich, and C. Wen, A Grassmannian etude in NMHV minors, J. High Energy Phys. 07 (2010) 061.

[105] S. Weinzierl, Tales of 1001 gluons, arXiv:1610.05318.

[106] N. Arkani-Hamed and J. Kaplan, On tree amplitudes in gauge theory and gravity, J. High Energy Phys. 04 (2008) 076 .

[107] M. Sgaard and Y. Zhang, Scattering equations and global duality of residues, Phys. Rev. D 93, 105009 (2016).

[108] J. Bosma, M. Sgaard, and Y. Zhang, The polynomial form of the scattering equations is an H-basis, Phys. Rev. D 94, 041701 (2016).

[109] Y. Zhang, Lecture notes on multi-loop integral reduction and applied algebraic geometry, arXiv:1612.02249.

[110] J. A. Farrow and A. E. Lipstein, From 4d ambitwistor strings to on shell diagrams and back, J. High Energy Phys. 07 (2017) 114.

[111] L. N. Lipatov, Graviton Reggeization, Phys. Lett. 116B, 411 (1982).

[112] L. N. Lipatov, Zh. Eksp. Teor. Fiz. 82, 991 (1982); [MultiRegge processes in gravitation, Sov. Phys. JETP 55, 582 (1982)].

[113] L. N. Lipatov, High-energy scattering in QCD and in quantum gravity and two-dimensional field theories, Nucl. Phys. B365, 614 (1991).

[114] L. N. Lipatov, Effective action for the Regge processes in gravity, Phys. Part. Nucl. 44, 391 (2013).

[115] L. N. Lipatov, Euler-Lagrange equations for the Gribov Reggeon calculus in QCD and in gravity, Int. J. Mod. Phys. A 31, 1645011 (2016).

[116] M. T. Grisaru, P. van Nieuwenhuizen, and C. C. Wu, Reggeization and the question of higher loop renormalizability of gravitation, Phys. Rev. D 12, 1563 (1975).

[117] A. Bellini, M. Ademollo, and M. Ciafaloni, Superstring one loop and gravitino contributions to Planckian scattering, Nucl. Phys. B393, 79 (1993).

[118] D. Amati, M. Ciafaloni, and G. Veneziano, Effective action and all order gravitational eikonal at Planckian energies, Nucl. Phys. B403, 707 (1993).

[119] D. Amati, M. Ciafaloni, and G. Veneziano, Towards an $S$-matrix description of gravitational collapse, J. High Energy Phys. 02 (2008) 049.

[120] S. B. Giddings, M. Schmidt-Sommerfeld, and J. R. Andersen, High energy scattering in gravity and supergravity, Phys. Rev. D 82, 104022 (2010). 